


\section{DISCLAIMER}

This report was prepared as an account of work sponsored by an agency of the United States Government. Neither the United States Government nor any agency Thereof, nor any of their employees, makes any warranty, express or implied, or assumes any legal liability or responsibility for the accuracy, completeness, or usefulness of any information, apparatus, product, or process disclosed, or represents that its use would not infringe privately owned rights. Reference herein to any specific commercial product, process, or service by trade name, trademark, manufacturer, or otherwise does not necessarily constitute or imply its endorsement, recommendation, or favoring by the United States Government or any agency thereof. The views and opinions of authors expressed herein do not necessarily state or reflect those of the United States Government or any agency thereof. 


\section{DISCLAIMER}

Portions of this document may be illegible in electronic image products. Images are produced from the best available original document. 


\title{
National Ursnium Resource Evaluation \\ URANIUM HYDROGEOCHEMICAL AND STREAM SEDIMENT RECONNAISSANCE OF THE ILIAMNA NTMS QUADRANGLE, ALASKA
}

\author{
Dete Complled by \\ Bendix Fiold Engineoring Corporation \\ Grand Junction, Colorado
}

\section{Data Colloction and Laboratory Analysis by}

Los Alamos National Laboratory

Los Alamos, Now Moxico

May 1981

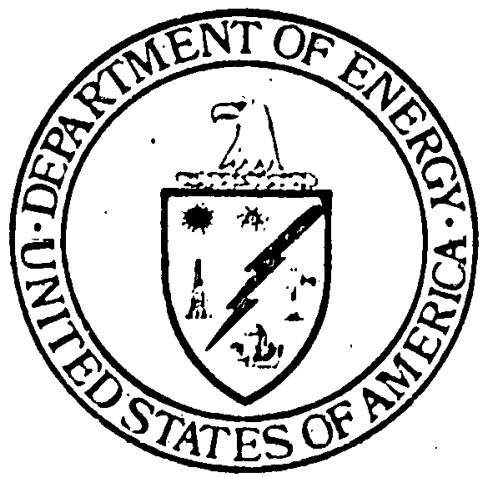

PREPARED FOR THE U.S. DEPARTMENT OF ENERGY Grand Junction Office, Colorado

Neither the United States Government nor any ansoned by an agency of the United States Government warranty. express or implied, or assumes agny tervereot, nor any of their employees, makes any

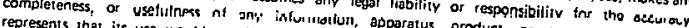

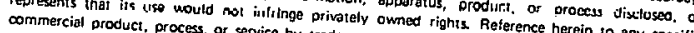

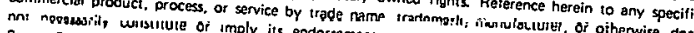

States Government or any or mply its endorsement. recommencation, or favoring by the Unito

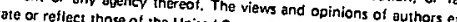


Neither the United States Government nor any agency thersot, nor any of tholr employees, mokes ary warranty, express or implied, or assumes any lagal liabillty or responsiblity for the accuracy, completeness, or usefulness of any information. opperatus. preduct, or prccess disclosed in thls report, or reprosents that its use would not infringe privatoly owned rights. Roterence thereln to any specifle commerclal product, precoss, or service by trade name, trademark, manufacturor, or otherwise. does not nacessarily constitute or Imply lis endorsement, recommendation, or favuring by the United States Government or any ogency thereot. The views ond opinions of authors expressed herein do not necessarlly rete of reflect those of the United States Government of any agency thereot.

Thle report lo o result of work performed as part of the Natlonal Uranlum Resource Evaluation. NUAE is a program of the U.S. Dopartmont of Energy's Orand Junction. Colorado. Office to ocquire and complle geologie and other information with which to assess the mognitude and distribution of uranium rosources and to dotermine aroas tavorable for the occurrence of uranium in the United States. 
URANIUM HYDROCEOCHEYICAL AND STREAM SEDIMENT RECONNAISSANCE OP THE

ILIAMNA NTMS QUADRANCLE; ALASKA

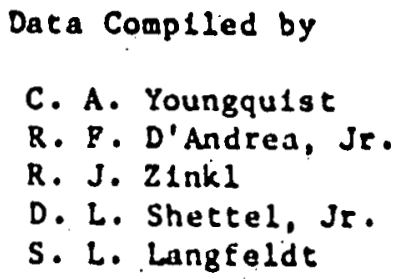

C. J. Thomas

C. J. Martel1

L. W. Maassien

LOS. ALAMOS NATIONAL LABORATORY Los Alamos, New Mexico

May 1981

PREPARED FOR THE U.S. DEPAKTMENI OP ENERGY GRAND JUNCTION OFFT,E, COLORADO 


\section{ILLUSTRATIONS}

Flgure 1. Uranlüm versus Thor1um, Stream Sediments . . . . . . . . 8

Flgura 2. Vanadium (ppm) In Stream Sediments . . . . . . . . . . . . . 9

Figure 3. Copper (ppm) in Struain Sedlinents . . . . . . . . . . . 10

Fligure 4. Uranlum versus Thorlum, Lake. Sedlmentg ............. .12

Flgurie 5. Vianadium (ppm) in Lake Sediments . . . . . . . . . . . . 13

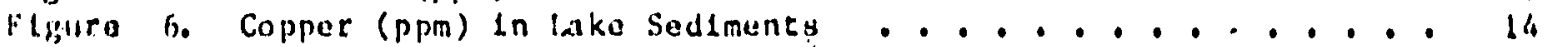

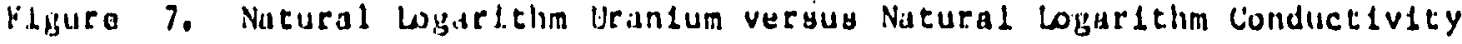
In Stran Water . . . . . . . . . . . . . . 15

Flinra b. Natural Lgarsthm Ueanlumi versus Natural Logartelim Conduetivity In Lake Watur .. . . . . . . . . . . . . . . . . 17

Flgura 9. Struan Sedlment factor Scoro 1 . . . . . . . . . . . . . 34

lisuru 10. Stream Sediment factor Score 2 . . ................. 35

Fligre 1. Stream Sediment factor Score 3 . . . . . . . ....... . 36

Fligura 12. Strean Sediment Pesiduals . . . . . . . . . . . . . . . 37

Flgure 13. Lake Sedlment Factor Score 1 . . . . . .......... . . 38

Figure 14. Lake Sedinent Factor Score 2 . . . . . . . . . . . . . . 39

Elgure 15. Lake Sedinent Factor Score 3 . . . . . . . . . . . . . 40

Flgure 16. Lake Sediment Residuals .... . . . . . . . . . . . . 41

Tible 1. Populacion Stacistics for Stream Sediment Data . . . . . . T

Tible 2. Population Statistics for Lake Sediment Data . . . . . . . . . 11

Tible 3. Regression Parameters, Uranlum versus Conductivity,

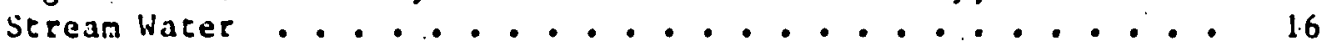

Table 4. Regression Paramecers, Uranfum veraus Conductivity,

Lake Watcer ... . . . . . . . . . . . . . . . . . 18

Tiblo 5. Stream Sediment Data Before Editing . . . . . . . . . . . . 20

rable 6. Streim Sediment Daca After Ed1fing . . . . . . . . . . . . . 21

Tible 7. Lake Sedlment Data Before Editing . . . . . . . . . . . . . 22

Tible 9. Like Sedtment Data dfter Edtting . .......... . . . . 23

Tabla 9. R-mode Factor dnalysis of Strean Sediment Data . . . . . . 24

Tablo 10. R-mode Factor Analysis of lake Sediment Data ........ . . 26

Truble 11. Kegression Analysis Results, Strean Sediment . . . . . . . 28

Table 12. Regression Analysis Results, Lake Sediment . . . . . . . . . 32 
Plate 1. Uranlum (ppo) in Stream Sediments............ . In pocket Plate 2. Thorium (ppm) in Strean Sediments............. . In pocker Plate 3. Thorium/Uranium in Stream Sediments............ . In pocket Plate 4. Streami Sediment Sample Locations. ............ . In pocket Plate 5. Uraniàn (ppa) in Lake Sediments.............. . In pockot plate 6. Thor 1uj: (ppm) In lake Sedinents.............. . In pocket Plite 7: Thorium/Uranium in Lake Sediments............. . In pocket Plate 8. Lake Sediment Sample Locatlons............... . In pocktet Plate 9. Uranlum (ppb) In Stream Water............... . . Ln lincket Plate 10. Urantum/Conductivity ln Stream Water. . . . . . . . . . In pocket Plate 11. Stream Nater Sample Locations............... . . In pocket Plate 12. Uranlim (ppb) in Lake Water................ . In porket Hate 13. Uranium/Conductivity in Lake Water. . . . . . . . . . In pocket Plate 14. Lake Water Sample Locations................ . In pocket 
This report presents results of a Hydrugeochenlcal and Stream Sediment Reronnalsgance (HSSR) of the Illamna VTIS quadrangle, Mlaska. In addtelon to thlt thbrovlited data relcase, more complete dara are avallable to the publlc ln machlne-readable form' through the Crand Junction oft lce intormit lon system (G.JOLS) at lak Ridge National Laburatory (ISRIL). Inquitles regarding the machlne-readable data should be directed to:

\author{
CJoIs Project \\ UCC-ND Compiting Applications Department \\ ¿500 North Bullding \\ Dak RIdge Nat Lonal Laboratory \\ P. 0. Box X \\ Oak RIdge, IN 37830
}

Presented In this data reiease are location ditil. Eleld analyses, and laburatory analyses of several different sample medla. For the sake of brevity, many tield st a observations rave not been included in this volume. These data are, howavar, avallable on the magnetlc tape. Appendices A to $D$ describe the samplo medla and sumarize the analytical resulty for each medlum. The data were subsetted by one of the Los $N$ amos National Laboratory (LANL) borting programs

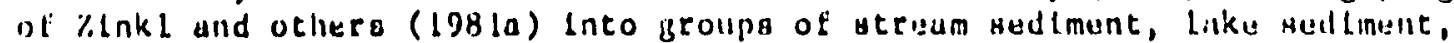
stritilll wilter, Like water, and ground water samples, for anch group whleh inilialine a sutflclent number of observations, statgeleal tables, tablas ot raw

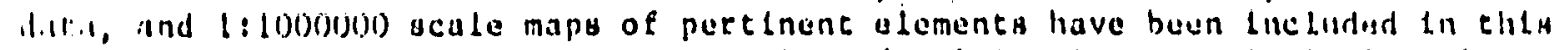

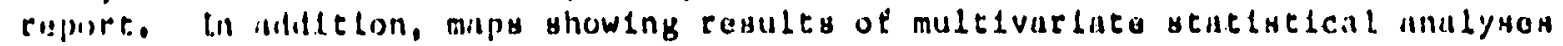
liave busn linc:Linded.

Thi IL Lamna NTMS quadrangle hydrogeochemlcal reconnalsannce was performed by the LANL, Inder iontract to the Gand Junction offlce of the Unlted States Dupartment of Energy (DOE), as part of the National Urantuin Resource Evaluntion ('li:?) ar irtm. MNI, was responstble for conducting the HSSR program in the i: ices of New Mexlco, Colorado, Wyoming, Montana, and Alaska. Thls data release was prispired by the Bendix Fleld Englneering Corporation in order to make the dita avallable to members of the public who wish to make use of the information. but who do not have access to computing facilities.

Further Infurmation about the HSSR progran in general, or about che ldNI portion ot tho program in particular, can be obtalned in quarterly or semiannual program progress reports on opentile at DOE's Technical Library in Gand Junction. linquirlus should be directed to:

Technical Library

Bendix Fleld Engineering Corporation

r. 0 . Box 1569

Grand Junction, CO 81502

Inturmation about the ileld and analytical procedures used by LivL during sample collection and analysis may be found in any HSSR data release prepared by the LaNL (see, for exariple, Planner and others, 1981), and w111 not be included in this report. 
Populatlon statistics for stream sediments are glven In Table l (pare 7 ) and fur lake sediments 1r. Table 2 (page 11). These tables concaln itatistlc:s such as mean, standard deviation, skewness, and kurtostis for tin ditterene populatelona: the total population and a population consisting of only those dilatyses thite ar. above the decection $11 \mathrm{mlt}$ ( $A D L$ ) for each element. In addlelon, data are provided for each varlable for the total number of sianplas pussussing anilysus,

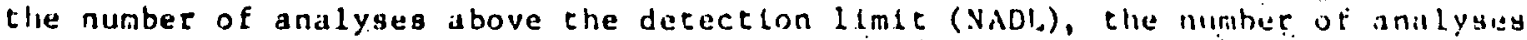
below the detection $11 \mathrm{mlt}$ (NBDL), and the number of missing analysey (:MlSS). The maximum value obtalned for each measurement is listed as well as the ininimum value for above detestion limit analyses and the maximum detection 1 inlt nbtalned. Varlable detection 1 lates account for the tact that the detection $1 \operatorname{lmlt}$ ls sometimes greater than the minlmum above detection limit value.

Maps of elemental concentrations, ratios, factor scores, and reslduils were prepared by a computer program which represents data values in thelr correct latitude-longltude positions by symbols which vary in size and intenelty in proportion to the value belng plotted (21nkl and others, 198/b). Mapr of urantum concentrations in stram sediment, Lake sediment, stronm watur, Hind lake water are presented as Plates $1,5,9$, and 12 , respectively. Thurluin concantrations in stream and lake sediment samplas and thorium-tomranlum ratios of tream and lako sediment gamples are ploted in plates 2, 6,3 ,

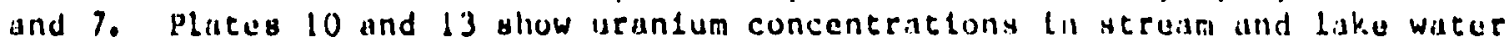

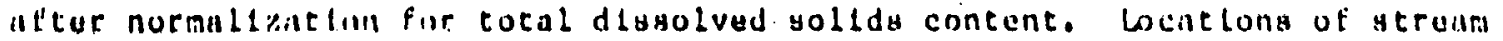
sedlmunt, lake Hediment, strean water, and lake witur amplus are shown in Plater $4,8,11$, and 14 .

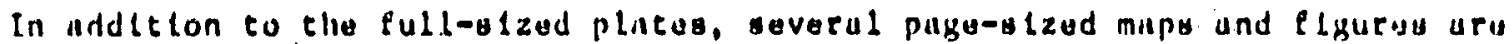
Inclided to assist in inturpectacion of the data. Scaccor ploty of iruntum

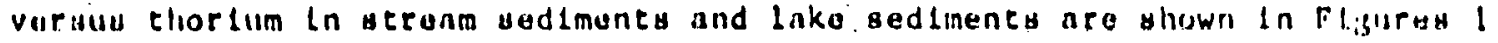
and 4. Thuse plots may be used to identily groups of samplus whleh ditfur H isniflcantly from normal crustal Th/U ratlos. Devlations from normal rat 108 may indicato areas showling urantum entichment or depletion. Stmilarly, 11 near rugresstons have been calculated (Tables 3 and 4 ) and plotted (Flguras 7 and b) tor uranium versus conductivity. These regression analyses may ladicate those samples in which a high uranium consent is present simply as a resule of large amuunts of total sollds dissolved in the water.

Maps of other elements whlch may be of value In uranlum exploration are included, when analyzed for by bavl. Flgures 2, 3, 5, and 6 ghow vanadlum In atruam sediments, copper In stream sedlments, vanadium in lake sediments, and coppor In lake sediments, respectively. 
THIS PAGE

\section{WAS INTENTIONALLY LEFT BLANK}


Tatle :. Population Statisties for Strean Sediment liata

one mese nacesesa

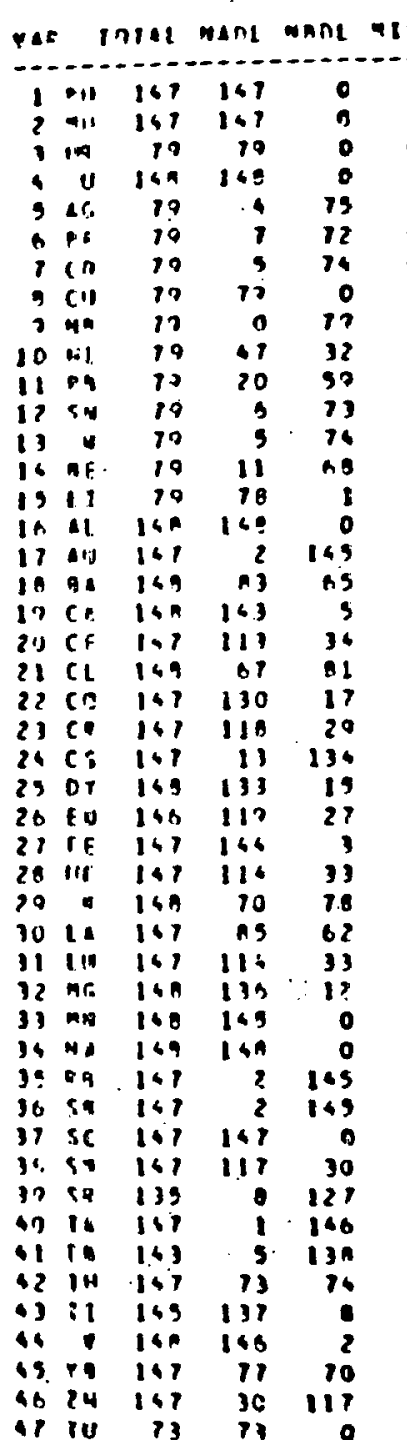

DFAN SIR.DFY. SOFUNFS RID INSI:

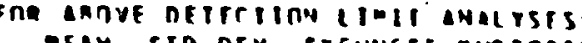

$1.93 \quad .6 ? \quad 3.00 \quad 21.69$

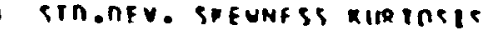

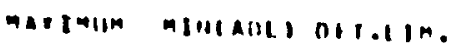

7.13

.82
01.30
2.84
1.35

3.00
3.44

51.46

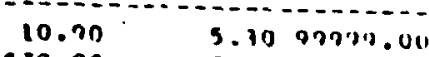

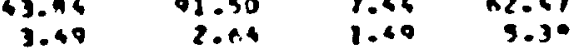

1.49 1.35

4.10 3.30
37.91

2.09

2.75

39.10

10.02

1.31

I.18

29.79

0.00
26.79

$5.03 \quad 29.33$

3.13

12.31

23.95

26.79

3.22

3.66

0.00

39.37

0.00

10.31

2.17 11.41

0.2

3.13

4.30

$20.70 \quad 12.03$
13205.08

3.84

21.14

10.33

$7058 \mathrm{Al1} 13295.0$

1.04
-1.64

3.96

430.42361 .34

$33745.22 \quad 17971.65$

23.16

3.10

4.51

45.11

.45
1.30

3.34

$17.9 ?$

4.60

$60.64 \quad 64.72$

$2.21 \quad 2.99$

$3.97 \quad 2.46$

66743.35 54460.97

138.4

$\begin{array}{rr}13.34 & 10.30 \\ .37 & .24 \\ 13440.02 & 947.92\end{array}$

$1258.07 \quad 537.21$

22704.36 IF5n.00

$\begin{array}{rr}39.71 & 39.100 \\ 2.12 & 3.39\end{array}$

19.90

2.95
253.04

7.11

1.80

253.04 113.A?

2.92

1.08

2.72

4732.51

228.30

3.39

3046.09

234.94

$3.00 \quad 3.23$

$2.24 \quad 12.93$

29.00

$9.94 \quad 60.08$

$3.00 \quad 36.99$

21.19
28.23

03.04

1.47

6.50

0.46

33.10

0.00

18.31
13.23

24.17

10.00

1.09
20.94

2.30

1.98

2.31
20.79

0.00

2n.5n

9.64

0.05
1.22

COOAn.11

11.9

1.40

$2.97 \quad 010.00$

2.00 2947\%.00

6.7917 .99

14.00
11.71

$0.00 \quad 1.92 \quad 12.00$

1.60

$.4 h$
$0.0 n$

0.00
2.20

i.11

a. 11

i.ns

15.213

3293.07
.01

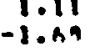

173.10

$33.22 \quad 22.02$

22.02
206.93

$10.72 \quad 11.79$

$15.15 \quad 60.10$

3.77

1.80

$\begin{array}{ll}3.29 & 28.23 \\ 3.29 & 36.28\end{array}$

36129.50

$4.3111 n 19.01 \quad 3105.09$

31.67

19.21
14.49

3105.09
10.13

10.13
.13

$4.62 \quad 30.23 \quad 0.33$

$\begin{array}{rrr}0.94 & 3.35 & 16067.21 \\ 1.44 & 1.74 & 1235.07\end{array}$

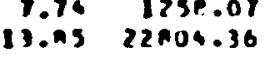

$2.73 \quad 1301$

6172.04

317.21

$25.30 \quad 36.00$

7950.00

$12.0 n$
.50

$\begin{array}{rrr}0.12 & 4.07 & 1.90 \\ .14 & 7.14 & 10.90\end{array}$

$\begin{array}{rrr}3.14 & 7.74 & 10.00 \\ 3.21 & 12.77 & 3.05\end{array}$

7.13

1.23

336.019

136.46

0.00
.00

1.70

$6.16 \quad 9.45$

3.2138 .41

3.25

10.94

30.92

970.93

3.46

$1074.9 A$

$220.56 \quad 239.69$

.00

.36

.61
3.21

3.21

2.15

3.44

.86
.46
.16
9.35

.35
3.01

.10

3.25

2.31
.14
1.68
2.23

2.23
0.00

0.00

.16
4.65

.99

0.00

$0.4 ?$

3.49

2.00

1.31

$1.52 \quad 10.00$

$3.10 \quad 11.0 n$

$\begin{array}{ll}3.55 & 150.0 n \\ 0.00 & 10.00\end{array}$

$0.20 \quad 193.00$

3.37

$\begin{array}{ll}31.00 \\ 1.02 & 19.00\end{array}$

$1.53 \quad 24.00$
$9.10 \quad 200$

$000 \quad 01.00$
0.51200000

-.51 110noo.00

l.or.

3.317950 .00

$17.20 \quad 162.00$

$\begin{array}{rr}19.38 & 97.00 \\ 13.62 & 93.40\end{array}$

$19.46 \quad 312.00$

$4.27 \quad 0.10$
2.00

2.00

2.9h 1.00

27.41401900 .00

2.19
$2.10 \quad 17720.00$

$419 \quad 95.00$

$17.53 \quad 1.10$
$3.96 \quad 343.0 .00$

$3.56 \quad 36510.00$

13. 3eshono

$1.00 \quad 67.00$

$1.00 \quad 2.00$

$2.16 \quad 41.30$

$\begin{array}{rr}13.20 \\ 3.0 n & 960.00\end{array}$

$0.00 \quad 4.0 n$

$\begin{array}{rr}3.25 & 2.00 \\ 90.0 ? & 30.20\end{array}$

$\begin{array}{rr}30.0 ? & 30.20 \\ 20.32 & 25010.00\end{array}$

$30.14 \quad 2003.00$

$\begin{array}{rr}9.91 & 9.3 n \\ 3.37 & 34.00\end{array}$

2.97

1.0027977 .00
$.40 \quad 92979.00$

$\begin{array}{rr}5.00 & 5.00 \\ 5.00 & 5.00\end{array}$

3.00
$3.00 \quad 9.00$

12.00 Mอกกด.00

$10.00 \quad 20.00$

$3.00 \quad 5.00$

$14.00 \quad 10.00$

$15.00 \quad 15.00$

$\begin{array}{ll}1.00 & 1.00 \\ 1.00 & 1.00\end{array}$

12170.0097929 .00

$209.00 \quad 437.00$

497.009741 .00

$11.00 \quad 39.00$

236.00

$10.00 \quad 4.70$

$1.20 \quad 2.60$

$1.00 \quad 9.00$

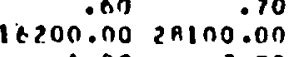

1.AO

$0.00 \quad 0.00$

4219.00 43.56.00

131.00979119 .00

3 i 7.0079979 .00

$49.00 \quad 37.00$ $\begin{array}{rr}1.00 & 3.00 \\ 2.40 & 29299.00\end{array}$ $1.10 \quad 3.90$ $327.00 \quad 114.00$

1.00

(1) $170 \quad 2.100$

35.00
$35 n n .00$
111.00

$\begin{array}{rr}1.00 & 2.00 \\ 31.00 & 0.010\end{array}$ .A 92707.00

ALIMAEO ni SAMPIFS = 140

1.73

.90

$--$ 
ILIAMNA STREAM SEDIMENTS

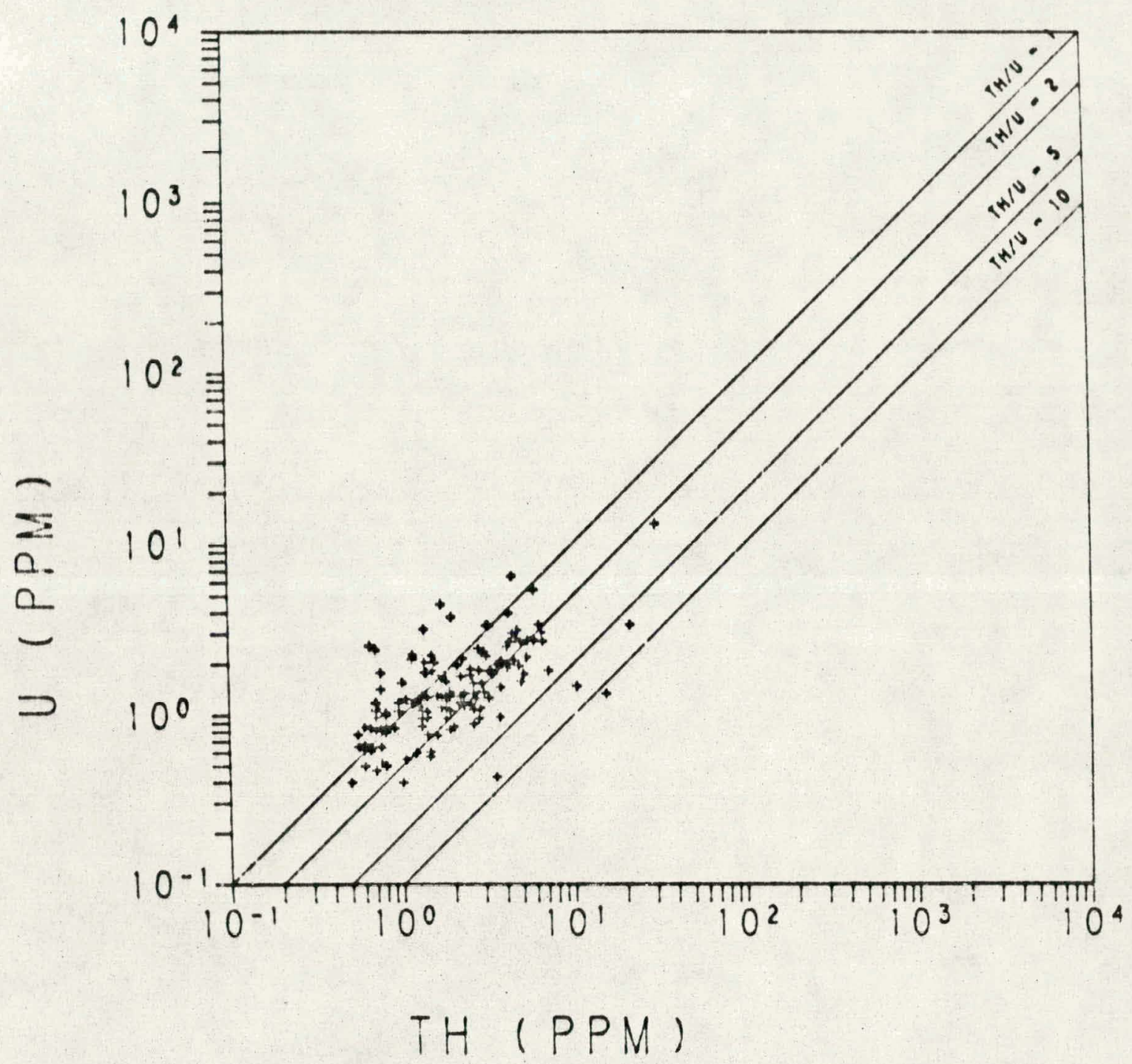

Figure 1. Uranium versus Thorfum, Stream Sediments 
IL IAMNA, AK: $V$ (PPM) IN STREAM SEDIMENTS

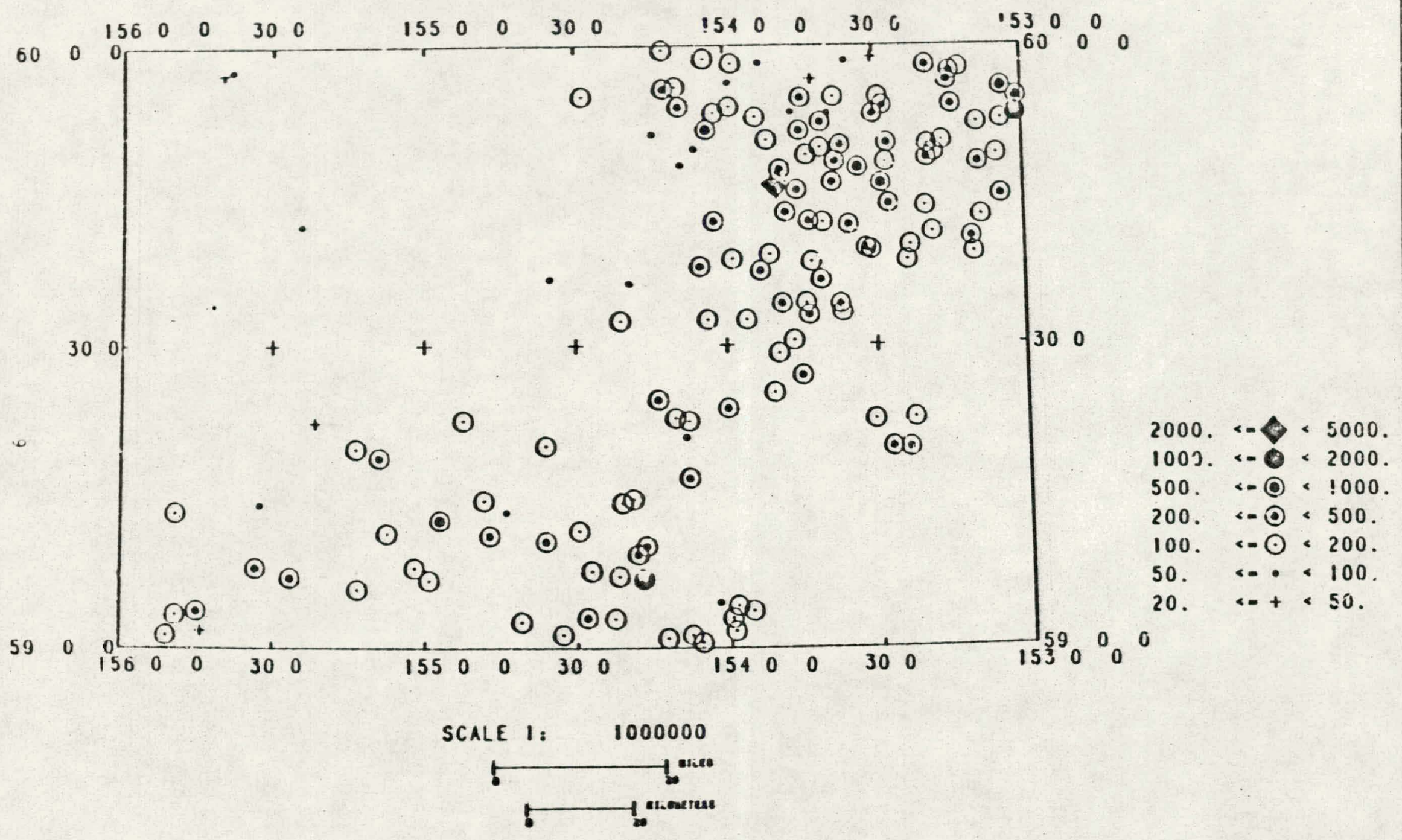

Figure 2. Vanadium (ppos) In Streas Sedimunts 
Table 2. Population Statistics for Lake Sediment Data

II IAMNA LAKF SERIMENIS

Fno inIAL ANALTCES:

MFAN PRODEV. SREUNFS QUETASIS

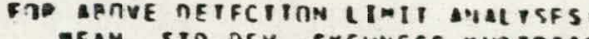

VAR TOTAL naDL waDe MIS

1 .4 $303 \quad 303$

$0^{0}$

\section{-}

7.09
34.55

$.20 \quad-1.62$

16.35

.35
.72
.00

4.21
0.60

0.00
1.75
2.50

2.24
0.00
.74

7.01

9.00

9.52

14.33
0.00

2.65

2.56
25.51

25.51
10.00

$\begin{array}{rrrr}10.00 & 0.09 & 0.00 & 0.00 \\ 12.06 & 11.39 & 3.37 & 17.43 \\ 4.55 & 4.49 & 4.09 & 41.18\end{array}$

.42

11.70

3.70
0.51

90.20

6.51
1.54 4.68

C11 211 205 92

$10 \mathrm{NI} 211$ 56 155

$\begin{array}{lllll}11 & P A & 211 & 37 & 164 \\ 12 & 54 & 211 & 6 & 205\end{array}$

$\begin{array}{lrrrr}12 & 5+4 & 211 & 6 & 205 \\ 13 & 4 & 211 & 2 & 209\end{array}$

$\begin{array}{rrrrrr}16 & B E & 211 & 37 & 174 & 92 \\ 15 & 11 & 211 & 211 & 0 & 92\end{array}$

is al 303 $303 \quad 0$

$\begin{array}{lllll}\text { If } & 11 & 303 & 3 & 300 \\ \text { IS } & \text { AA } & 303 & 101 & 112\end{array}$

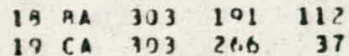

20 CF $303 \quad 202 \quad 101$

$\begin{array}{rlrrr}21 & \mathrm{CL} & 303 & 243 & 60 \\ 27 & \mathrm{CH} & 302 & 177 & 125\end{array}$

$23\left(\begin{array}{llll}130) & 136 & 167\end{array}\right.$

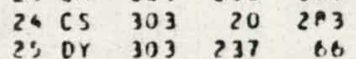

$\begin{array}{lllll}26 & \mathrm{FII} & 303 & 202 & 101\end{array}$

$? 7 \mathrm{FE} \quad 303290$

$\begin{array}{lllll}\text { ?A } & \text { IIF } & 303 & 182 & 121 \\ 22 & K & 303 & 17 \mathrm{~h} & 127\end{array}$

$301 \mathrm{~A} 303 \quad 126 \quad 177$

iI LII 303 17月 123

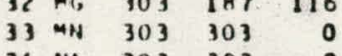

$\begin{array}{llll}34 & \mathrm{ma} & 303 & 303\end{array}$

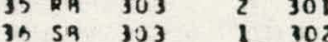

$37 \mathrm{SC} 303 \quad 302 \quad 1$

$\begin{array}{lllll}19 & 54 & 303 & 204 & 99 \\ 5 & 58 & 291 & 3 & 278\end{array}$

40 :- $303 \quad 2 \quad 301$

$\begin{array}{lllll}41 & 19 & 303 & 6 & 297\end{array}$

43 II $303 \quad 220 \quad 3$

$\begin{array}{lrrrr}64 & V & 103 & 2 A 4 & 19 \\ 45 & 19 & 103 & 114 & 199\end{array}$

SA IN 303 S5 $25 \mathrm{H}$

$\begin{array}{llllll}4+ & 7 N & 303 & 65 & 25 & \\ \rightarrow 111 & 146 & 146 & 0 & 15\end{array}$

mimare dif SAMMifs - JC3

2.71

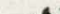

1.52

16.29

120.54

$\begin{array}{rr}12.16 & 153.33 \\ 3.20 & 16.15\end{array}$

10.63 21355.24

.0 A

$240.2 ?$

21.53
472.09

5.53

5.72
35.25

$35.01 \quad 35.25$

$2.17 \quad 1.61$

3.46

1.57

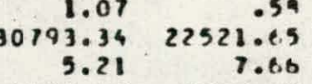

9307.68

13.37

9849.86

134.02

11.10

11.10

19100.07

6285.11

คด

14.15
3.15

$\begin{array}{rr}.51 & 9.59 \\ 5.11 & 35.29 \\ .46 & 3.23\end{array}$

4

3.23
20.45
21.37

$\begin{array}{ll}3.19 & 21.37 \\ 1.43 & 0.36\end{array}$

1.45

16.34
16.45

$3.17 \quad 17.95$

$3.23 \quad 18.36$

$3.11 \quad 21.52$

$5.30 \quad 45.21$
$7.41 \quad 60.25$

$03 .-4$

$3.32 \quad 19.85$

1.98

11.55
42.26

$\begin{array}{rr}5.10 & 42.24 \\ -.19 & 3.06\end{array}$

$4.02 \quad 40.59$
1.72

10.73

40.59

$-.15$

27.98

$5.21 \quad 42.22$

4.22
7.29

$\begin{array}{rr}2.78 & 1.59 \\ 241.30 & 55.83\end{array}$

1.04

1.66

1.35

9.30

$1.25 \quad 4.39$

33.94

०त3.97 2976.07

$\begin{array}{rr}0.93 & 3976.07 \\ 0.57 & 70.00\end{array}$

5.A3 l.

$\begin{array}{rr}4.41 & 32.27 \\ 2.18 & 17.47 \\ 4.07 & 20.09\end{array}$

27.95

0.47
3.29
4.92

1.96

.5 .3

$\begin{array}{rr}3.90 & 62.43 \\ .14 & 3.43\end{array}$

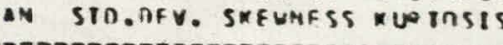

$\begin{array}{rrrr}7.09 & .20 & -1.62 & 16.36 \\ 36.55 & 42.52 & 7.01 & 77.72\end{array}$

$7.017 \% .7$

0.00
14.33

1.76

0.00

0.00

6.00
5.20

5.20
26.21

0.00

24.93

0.94

22.00

1.16

0.03
$? .024$

1.05

11.32

0.00

0.52

$0.0 n$
.57

1.50

1.79

14.55

2.33

4.24

10.51

.37

12.02

-48
-50

120.75

$37.64 \quad 9$ hoo.93

34.93

57.74

452.60

5.25

$4.71 \quad 1.24$

$\begin{array}{ll}.25 & 1.35 \\ 1.28 & .52\end{array}$

$\begin{array}{rr}1132.97 & 22474.46 \\ 7.19 & 9.29\end{array}$

7.19

18.32

10.99
10.94

090.04

136.02

3941.26

ถ०००. คด

180.07

7755.17

3.00

3.00

10.76

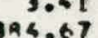

3.44

1.42

4.50

1.33

2.50

1.4?

1.03

.99

.50
-.39

3.91

4.27

1. Ah

3.81

.09

1.36

3.49

A. 31

.71

3.12

2.92

3.10

0.18

0.00
0.00

1.37

2.41

0.07

0.00
.71

$3 \times 57.74$

57.74

1.75

3. B

4.61

$3.59 \quad 79.57$

159.44
1.91

127.97

2.77

14.33
0.60
1.99

1.99
3.29

3.25

9.23
0.00

.78

26.31

3.39

$\begin{array}{ll}1.00 & 28.00 \\ 4.30 & 2.00\end{array}$

9.59205600 .00

1.50
$6.44 \quad 1639.00$

$6.44 \quad 1629.00$
34.20114100 .00

$\begin{array}{rr}27.53 & 189.00 \\ 9.57 & 3441.00\end{array}$

$27.16 \quad 51.00$

$4.68 \quad 270.00$

7.60

3.70

45.อ5 23 3 200.00

05.10
3.28

$21.94 \quad 92.00$

$\begin{array}{rr}11.11 & 1.10 \\ 17.31 & 30630.00\end{array}$

42.26 hE 16.00

3.6454750 .00

$1.00 \quad 42.00$

$\begin{array}{rr}0.00 & 3.00 \\ 7.35 & 40.10\end{array}$

$12.26 \quad 11.00$

402.00

$1.50 \quad 2.00$

$25.30 \quad 14.70$

$32.96 \quad 25600.00$

$131.43 \quad 1189.00$

$11.10 \quad 10.40$

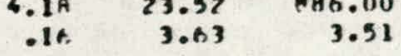

3.51

(ANI) DFT.IIM

5.0099999 .00

0.0023090 .00

, .2399997 .00$

$\begin{array}{ll}2.50 & 5.00 \\ 5.00 & 5.00\end{array}$

$\begin{array}{ll}5.00 & 5.00 \\ 5.00 & 5.00\end{array}$

$10.00 \quad 10.00$

$\begin{array}{ll}10.00 & 20.00 \\ 15.00 & 15.00\end{array}$

$5.00 \quad 3.00$

$11.00 \quad 10.00$

$16.00 \quad 15.00$

1.0094999 .00

7941.0092999 .00 $277.00 \quad 240.00$

$546.00 ? .6 .65 .00$

$15.00 \quad 15.00$

$116.00 \quad 460.00$

$2.50 \quad .10$
$20.00 \quad 21.00$

$2.00 \quad 3.20$

$1.00 \quad 4.00$

6 $320.00 \quad 521.00$

$\begin{array}{rr}2.40 & 1.90 \\ 776.00 & 56.05 .00\end{array}$

$6.00 \quad 12.00$

$2572.00 \quad 3297.00$

59.0079990 .00 2153.0090999 .00 $34.00 \quad 65.00$

$3.00 \quad 4.00$

$1.50 \quad 2.20$

$358.00 \quad 437.00$ 
ILIAMNA LAKE SEDIMENTS

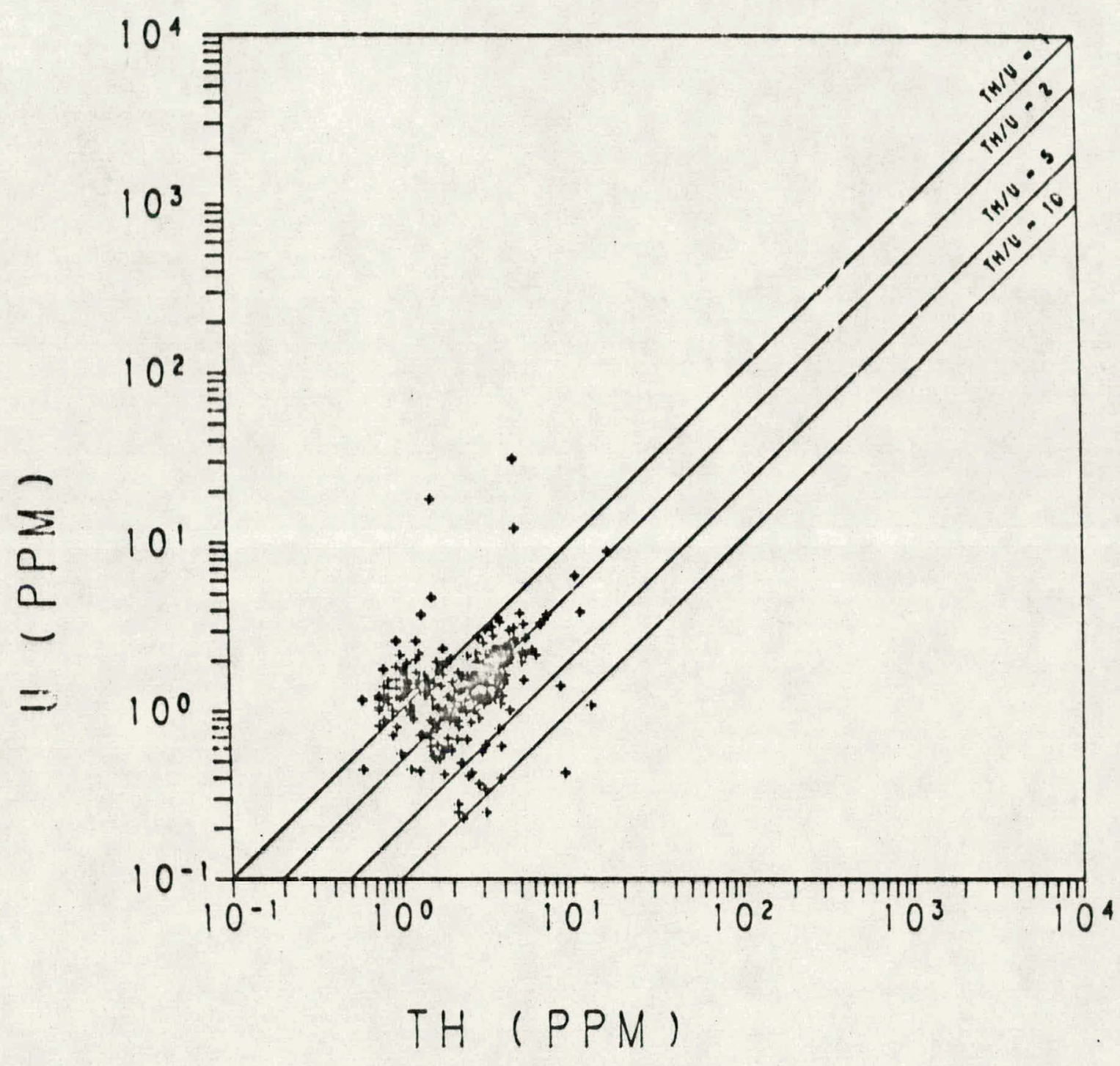

Figure 4. Uranium versus Thorfum, Lake Sediments 
IL IAMNA, AK: $V$ (PPM) IN LAKE SEDIMENTS

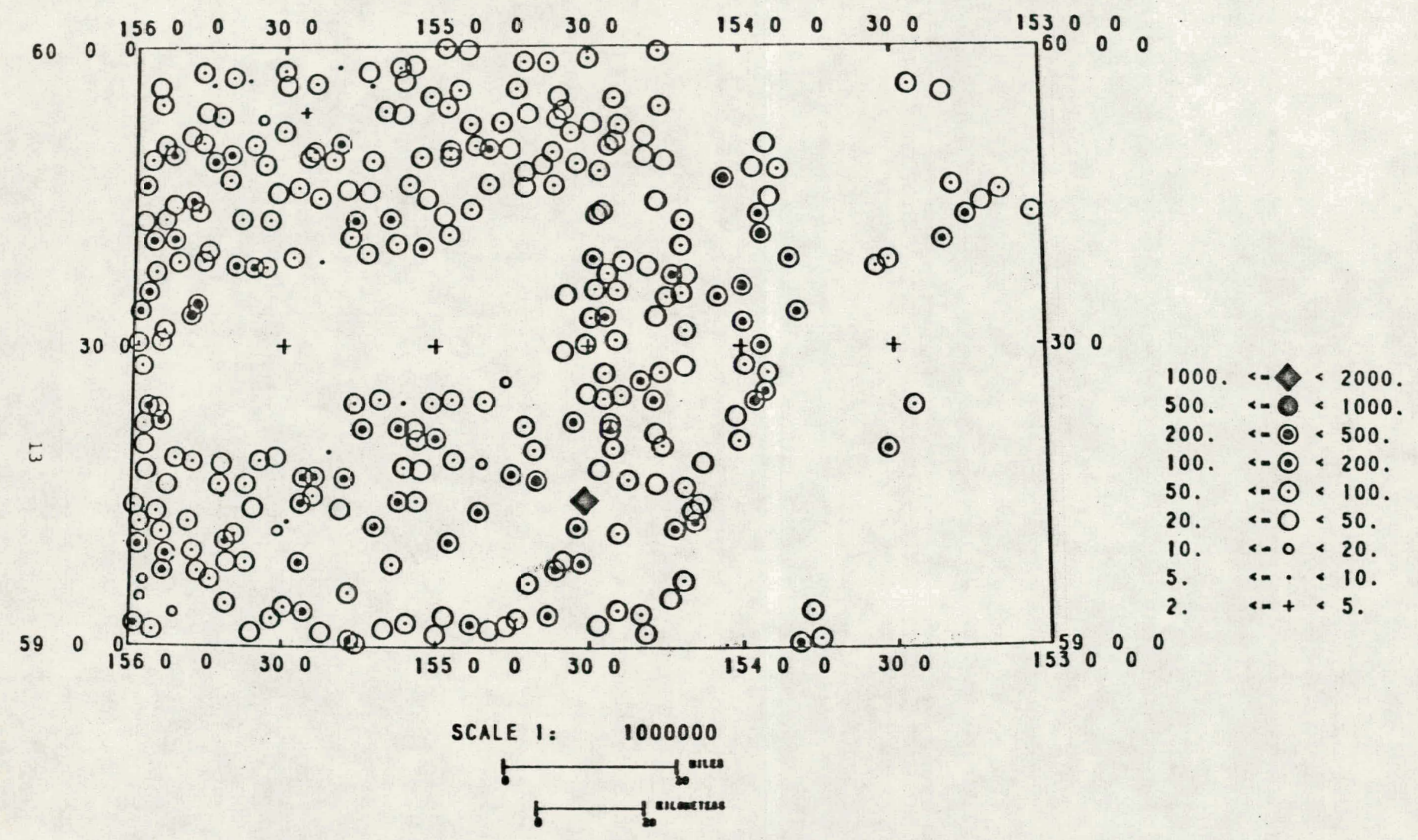

Figure 5. Vanadium ( $\mathrm{ppm}$ ) in Lake Sediments 
ILIAMNA, AK: CU (PPM) IN LAKE SEDIMENTS

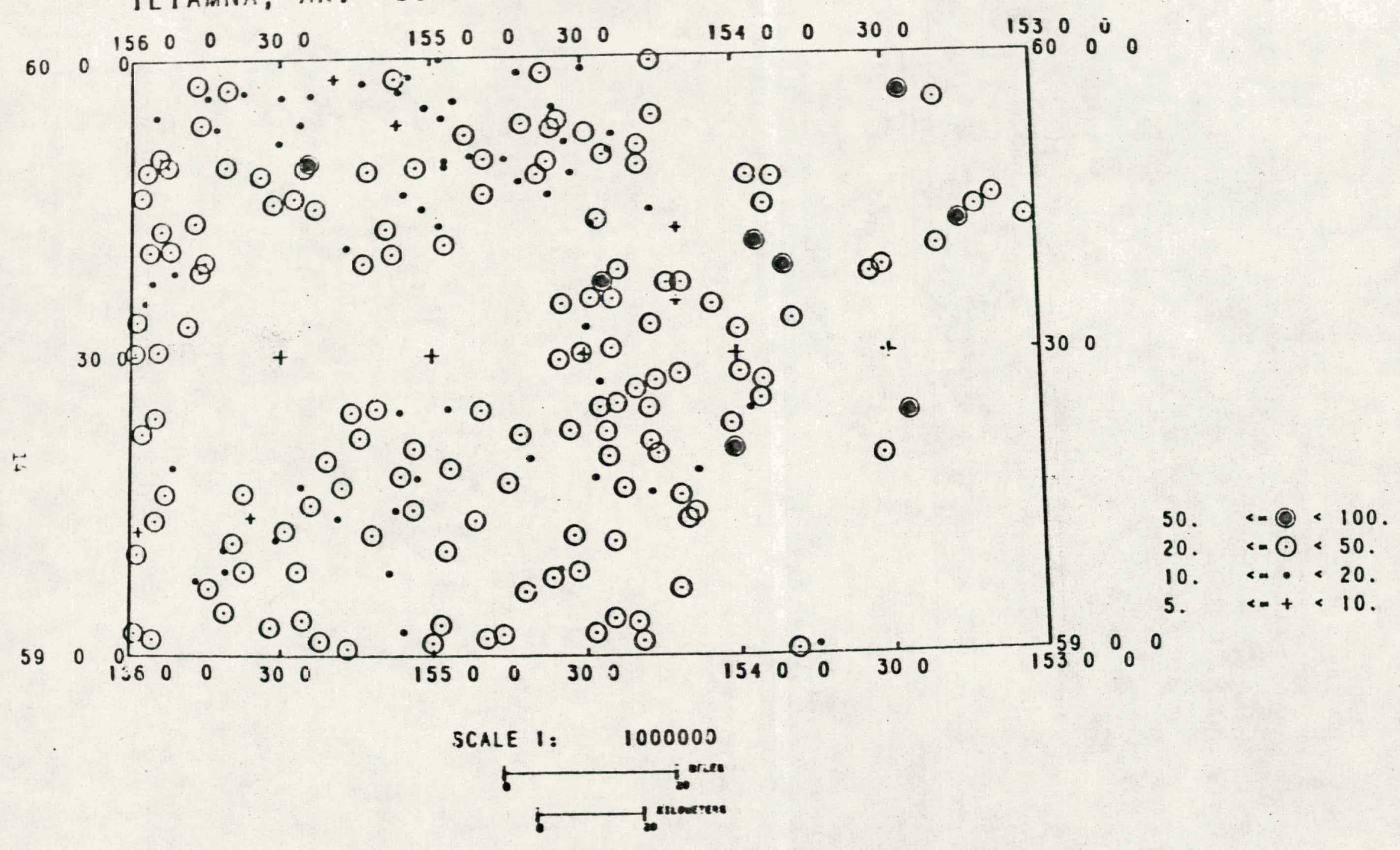

Figure 6. Copper (ppa) in Lake Sediments 


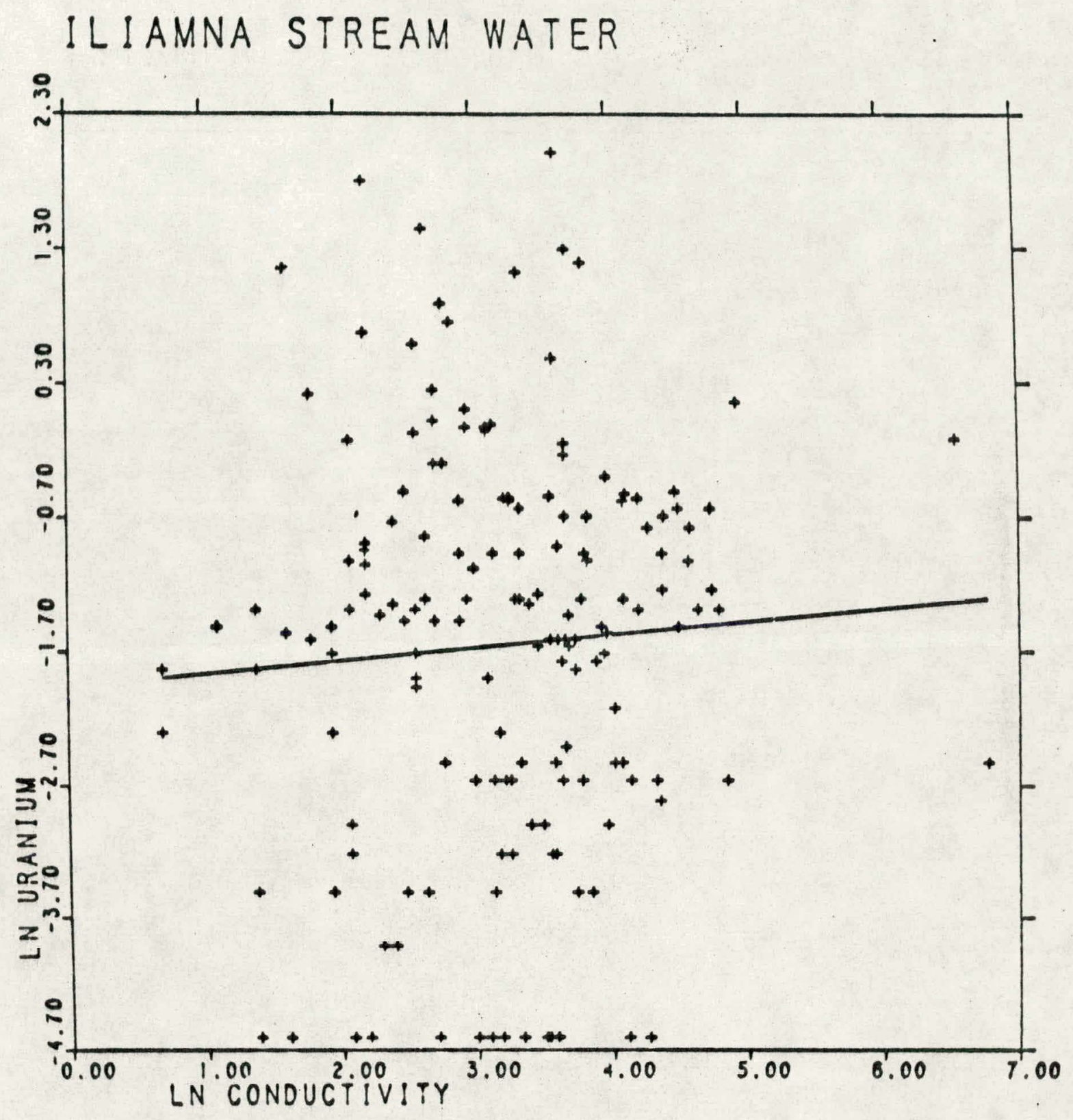

Figure 7. Natural Logarithm Uranium versus Natural Logarithm Conductivity in Stream water 
Table 3. Regression Parameters, Uranfum versus Conductivity, Stream Water

ILIGMNA STOEQM WATEO

SLCDE

.09727

INTEPCEOT:

$-1.9+299$

COQQELATION ENEFFICIFNT:

- C\&? C?

COFGF!P!ENT OF VAPIATITH:

.20197

COVARTANCE,

.09327

PMTAL SIM OF SगIAREC

$372.600=6$

TFSITUAL PIIM TF STUARES

279.99194

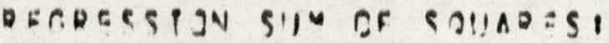

1.47900

STANADOR EOUEO TF D

$.190+7$

PORAAPILPTY THAT SLADE IS NFT LRTTY:

1.20000

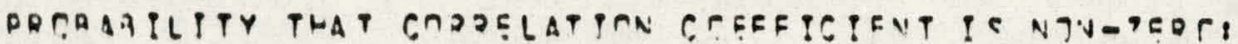

ESTha

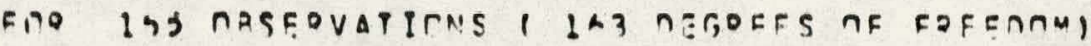




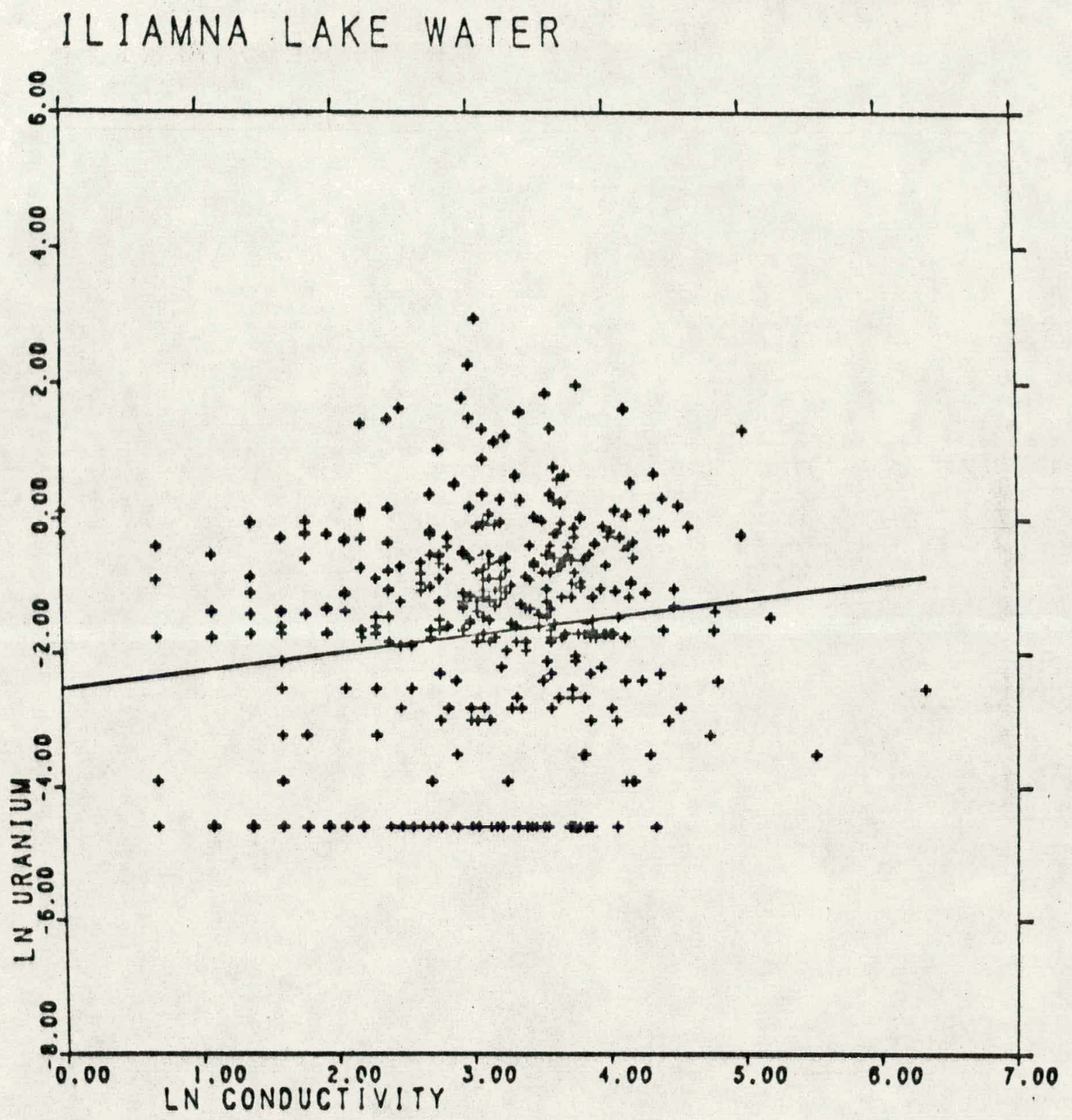

Figure 8. Natural Logarithm Uranium versus Natural Logarithm Conductivity in Lake Water 
Ts.ble.4. Regression Parameters, Uranlum versus Conductivity, Lake Water

ILTAMAA LAKE WATFo

SLCP:E

INTFOCEDT

CODQELATICN TOEFFICIFNT:

COEFETPIFAT TE VAOTAT? INA

CRVAOPANCE, I

$.2147 \mathrm{C}$

TCTAL TIIU TF SOI.AOFSI

DFETAIIAL SUM TE SOHAOES:

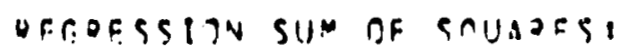

SPAHAADT EOQRO PF NI
.1369

$.0,9053$

$1116.0 \% 42 ?$

1905.14799

?1. +1 $1+29$

. ) วดรก

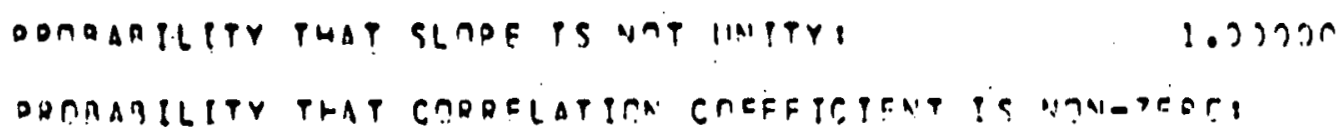




\section{MULTIVARIAIE STATISTICAL ANALYSES}

Stream-sediment and lake-sediment analyses were edlced by che PACEDT computer program. (Shettel and others, 1980) in preparation for multivarlate statstical analyses, by eliminating samples and variables with insufflclent data.

Information contalned in the stream-sediment and lake-sediment data sets bofore and after editing are shown in Tables 5 through 8 . The edited data sets are logaritnmicaliy transformed prior to the application of statstical methods.

$R$ mode factor analys 18 (Tables 9 and 10) was performed on the transformed edited -odiment data. R-mote compares varlables over the range of samples; Qtoode compares samples usir.g the varlables. Factor analysis attempts to show the geochemiral relationslips that exist in the data by reducing the dimenslonality of the multivarlate observations. (1.e:, by groupling varlables that ara highly correlated into "factors"). In the sediment data, urantum typlcally louds with Ce, La, Fe, Mn, $V$, and Sc, which 18 characterlatic of monazlte, z1rcon, the wagnetita-1imentte sulte, and the Irontanganese oxides famllles of atneralo. Results of factor analysis of ground-water data usually show urantum londing with the mulor lone, conf 1 rming the relationship between urantum and specif $1 \mathrm{c}$ cunductancu.

Becuusc of the hlgh degreu of correlation betwoen uranium and thoso elumunte us soclated with resistate minarals, stepwige multiplo ragreselon (Tablos 11 and 12) was employud to "correct" the raw uraniug value tor thist whlch is prount in heavy or reglatate rolniaralo. A map of the ragrusaton rusiduals shows those arous whure urantum cannot bu axplalnad by tho occurronce of rualetato or

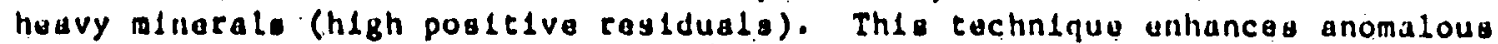
- aluplau by gupprasalng background populationg rualeing from rubletato or haavy minural.. Factor score and resldual maps are shown In Plgures 9 through 16. 
Table 5. Stream Sediment Data Before Edlting

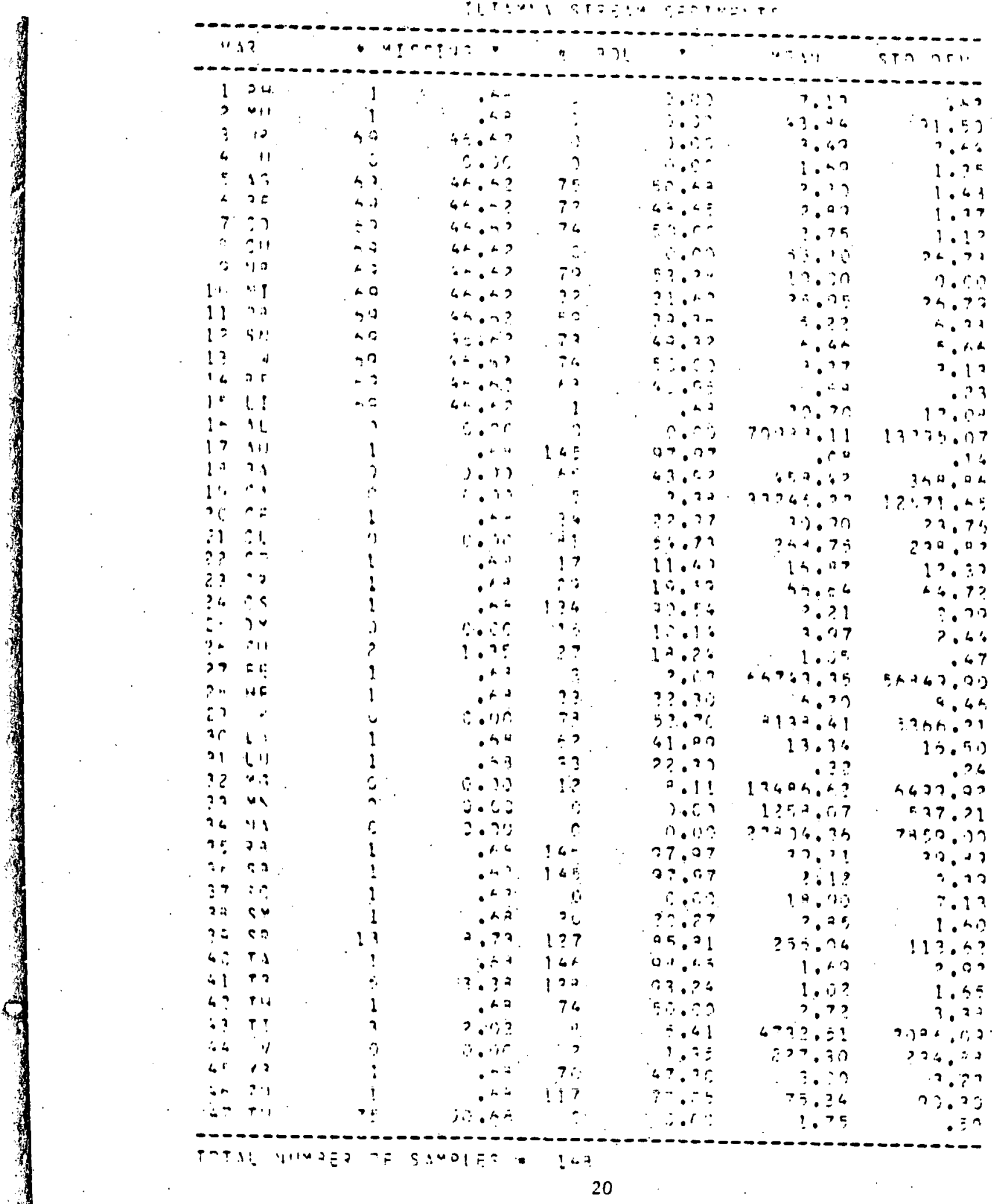


Table 6. Stream Sedlment Data Tir Ed1ting

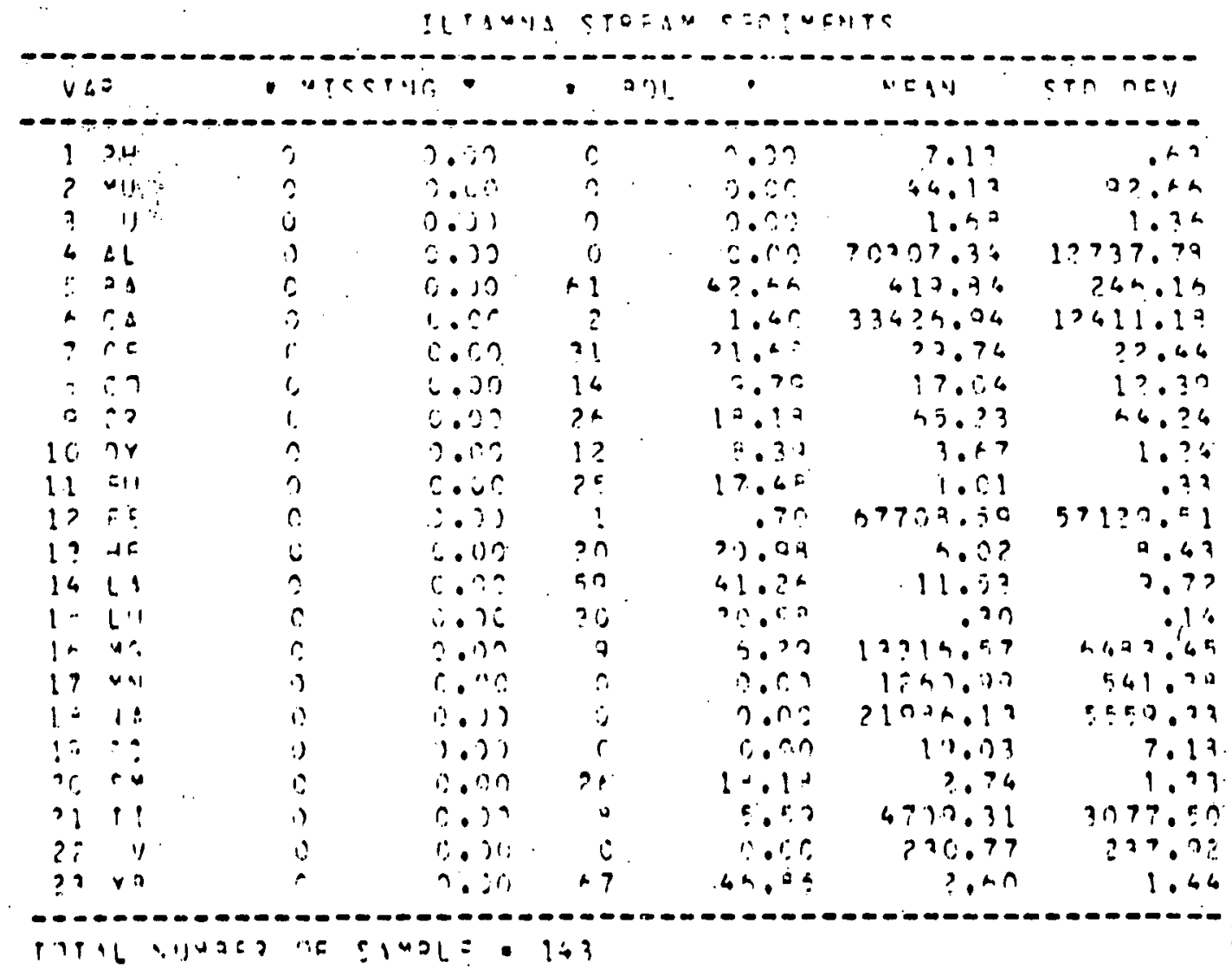


Table 7. Lake Sediment Data Before Editing

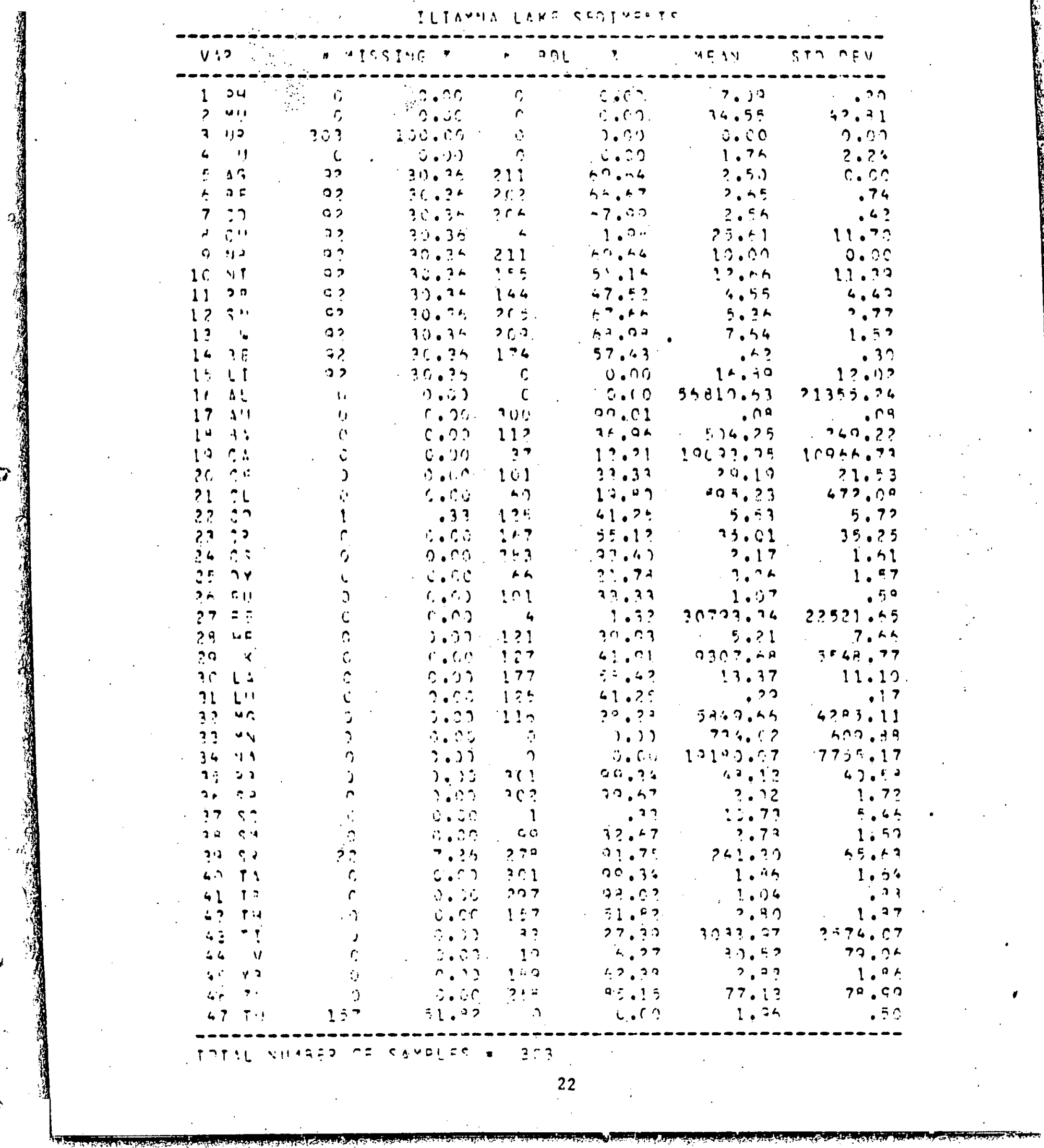


Table 8. Lake Sed1ment Data After Ed1ting

ILIANAA LANE SERTHENTS

\begin{tabular}{|c|c|c|c|c|c|c|c|}
\hline \multicolumn{8}{|c|}{$---\infty-\infty \cdot+--\infty-\infty$} \\
\hline 1 & 24 & 2 & 0.0 .0 & 0 & $r .00$ & 7.30 & $\therefore 20$ \\
\hline$?$ & ul! & $\eta$ & 0.00 & 0 & 0.00 & 34.52 & $4 ? 097$ \\
\hline$\xi$ & ' & $?$ & $\therefore .30$ & C & 3.00 & 1.75 & $? .26$ \\
\hline 4 & $\Delta L$ & c & 0.35 & 0 & $\because .02$ & $5 \leftarrow 779.00$ & $? 132 ? .09$ \\
\hline 5 & 94 & $c$ & 0.00 & 112 & 37.0 .2 & $5 ? ? .29$ & $? 4 a .0 h$ \\
\hline 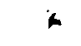 & $?: 1$ & $c$ & $\therefore .00$ & 37 & $1 ? . ? 5$ & $1311 ? .14$ & 10200.21 \\
\hline 7 & $\because \subsetneq$ & 0 & 2.00 & 101 & 33.46 & $? 0 . ? ?$ & $? 1.5 \mathrm{~h}$ \\
\hline 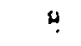 & CL & $c$ & $C \cdot S u$ & $+n$ & 10.57 & $54 ? .21$ & 44.2 .54 \\
\hline 2 & 67 & 3 & 2.0 .0 & $1 ? ;$ & 41.27 & $5.5 ?$ & $5 . ? 2$ \\
\hline 10 & $n r$ & o & s.CC & $+h$ & $2 ? .65$ & 2.95 & 1.53 \\
\hline 11 & F.1 & 2 & $C .: 0$ & 101 & $.33 .4:$ & $1 .: 17$ & .9 \\
\hline 12 & $=F$ & 0 & $0.0,0$ & 4 & $1.7 ?$ & 30 ค? ?. 70 & $? ? 949.72$ \\
\hline 12 & UF & $n$ & 0.09 & :?! & $4 j .1 .7$ & 5.81 & 7.49 \\
\hline 14 & $\kappa$ & $c$ & 0.2)$. & $1 ? 7$ & 47.65 & $0 ? 40.00$ & 5412,50 \\
\hline $1:$ & $6 !$ & 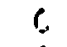 & $=0.1,0$ & $17:$ & 41.39 &.$? 2$ & .14 \\
\hline $1 \%$ & M: & $c$ & r.or & $11^{h}$ & 34.41 & 51950.57 & $4 ? 92 . ? 3$ \\
\hline 17 & $w n$ & J & $0.0 \%$ & 0 & serer & $? ? 6.24$ & $410 .+7$ \\
\hline LN & YA & : & 0.00 & $?$ & 2.0 .0 & 1.9141 .70 & 7970.27 \\
\hline 19 & ir. & ) & c...in & 1 & .73 & 10.72 & 0.47 \\
\hline$? 0$ & sn & c & $1) .1 .5$ & nn & 2.74 & $? .77$ & $1 .: 0$ \\
\hline 21 & $\varphi P$ & 7 & 0.96 & 02 & $7 ? .40$ & $33+7.09$ & 7.97 .94 \\
\hline$? ?$ & y & $n$ & 0.10 & 10 & 1.90 & 0.47 & 70.13 \\
\hline
\end{tabular}




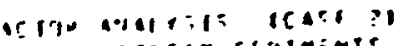

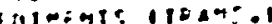

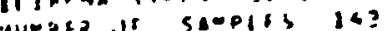

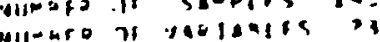

Table 9. R-mode Factor Analusis of Stream Sediment Data

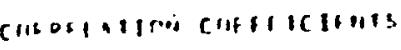

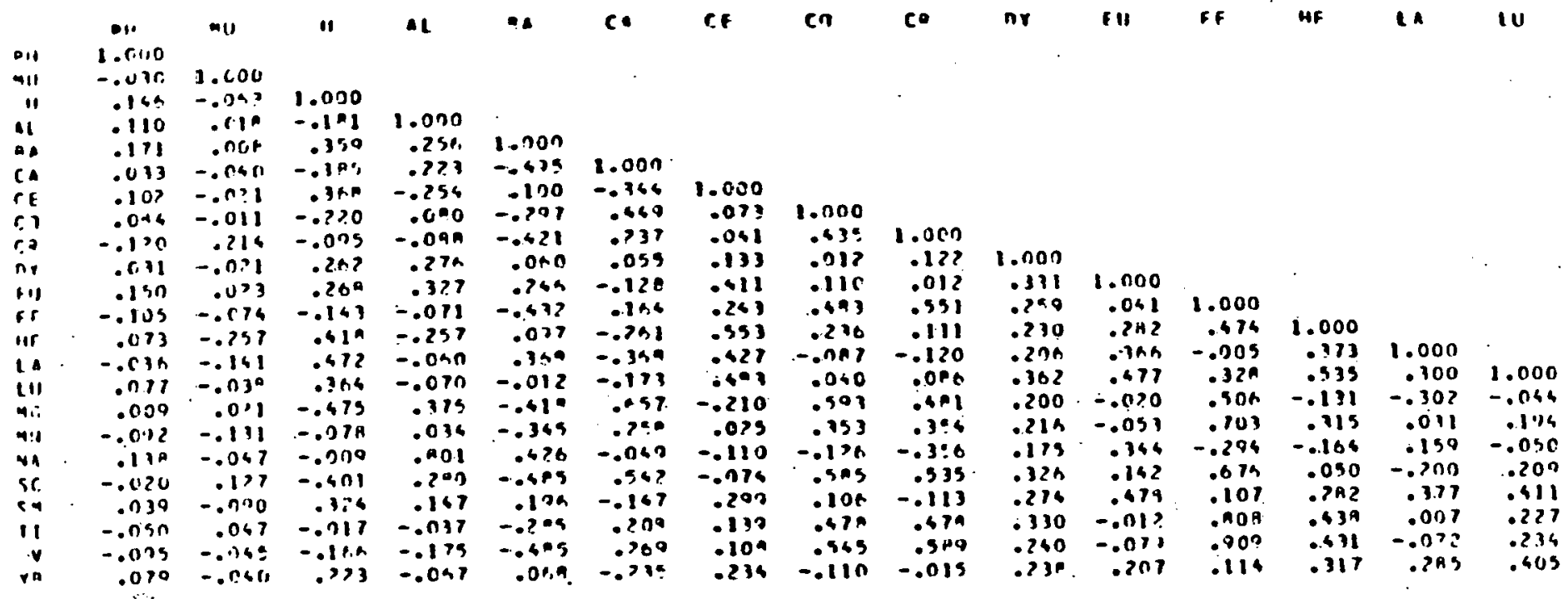

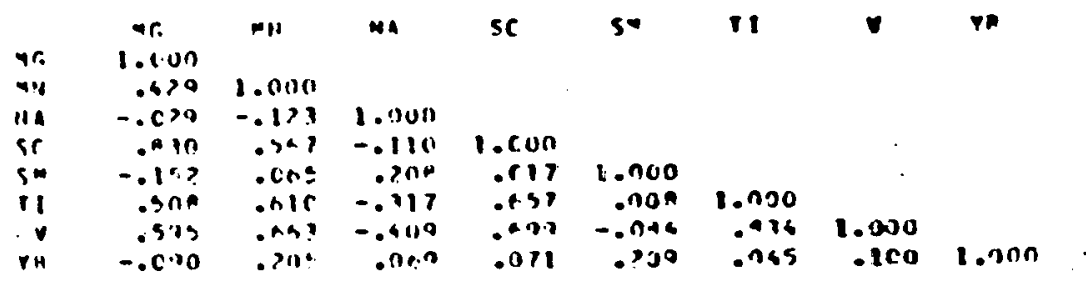


Table 9. R-mode Factor Analysis of Strean Sediment Daca(contirued)

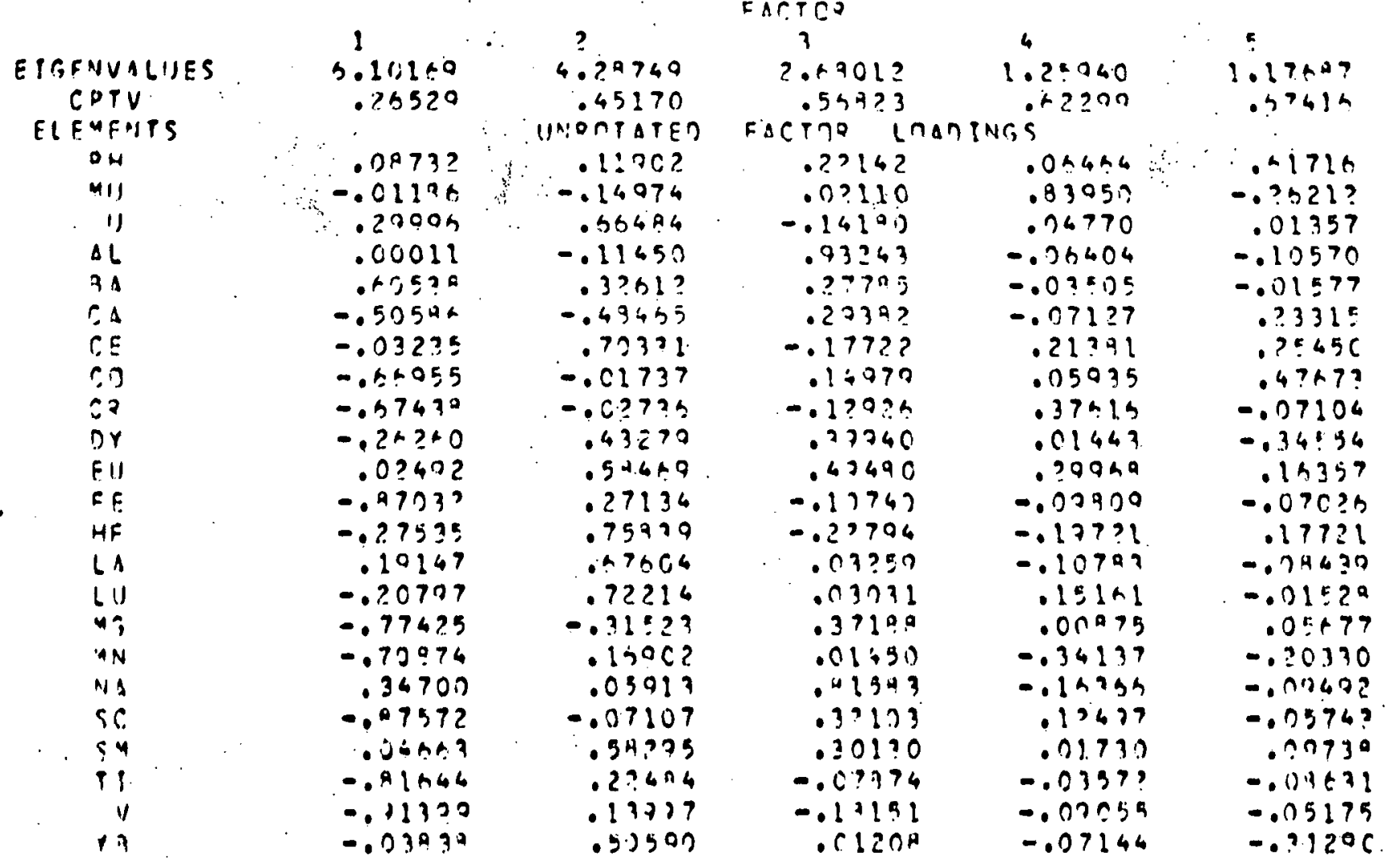




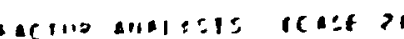

$4.9=3$

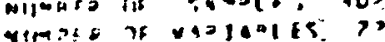

Table 10. R-mode Factor sal :sis of Lake Sediment Data

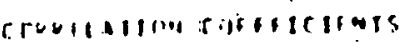
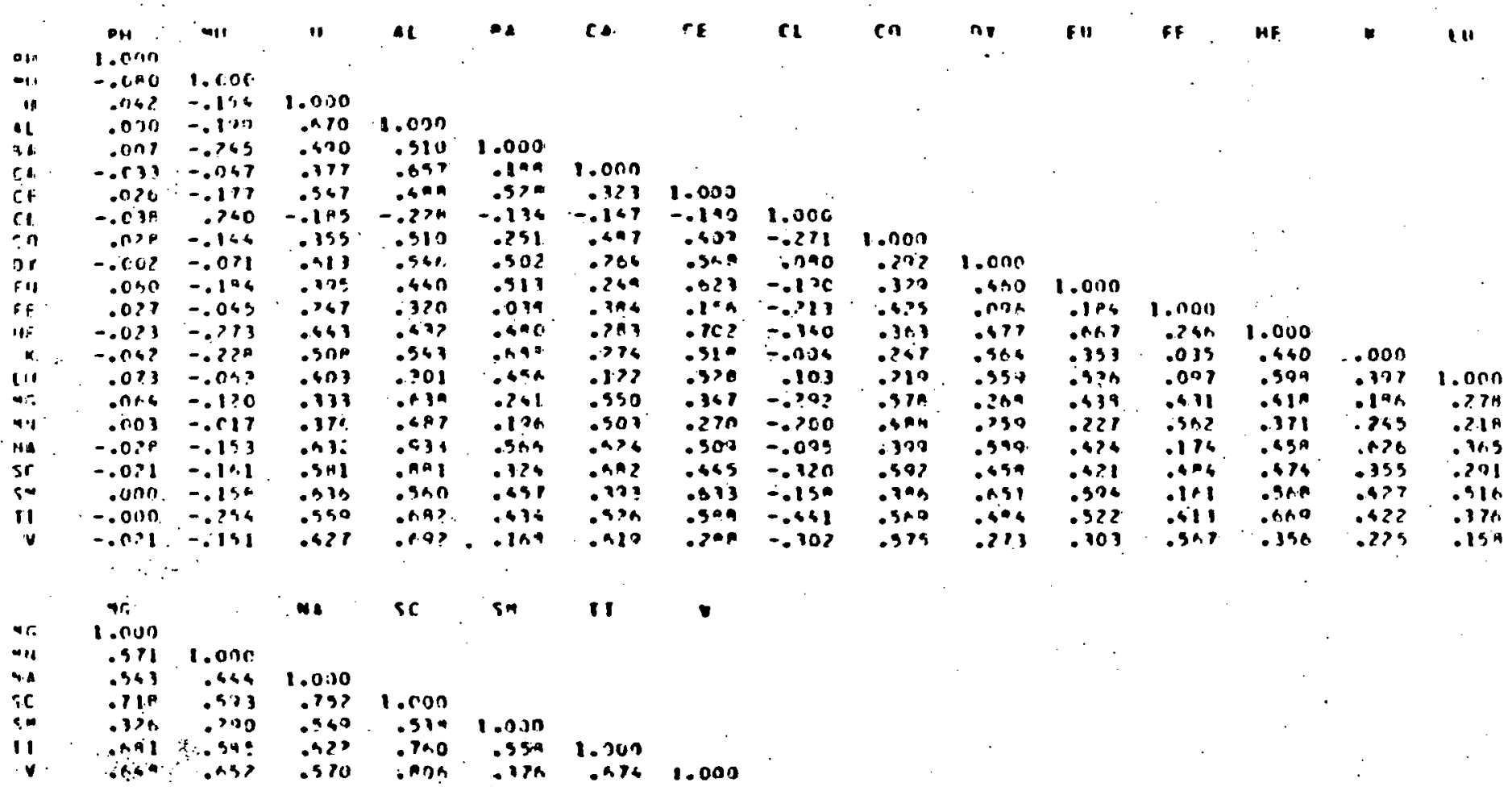
Table 10. R-mode Factor Analysis of Lake Sedinent Duta(cunitinued)

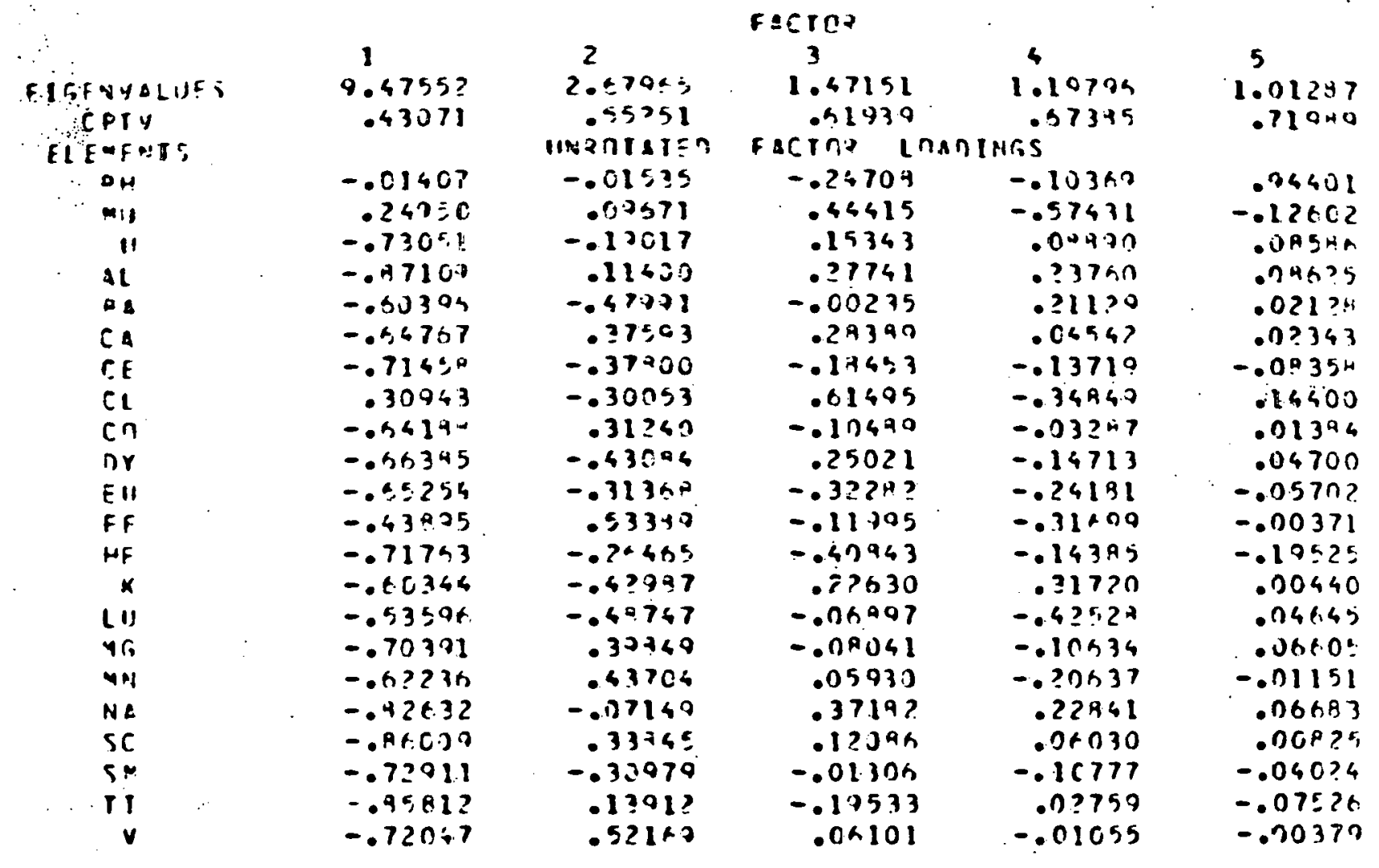


Table 11. Regresgion Analysis Results, Stream Sediment

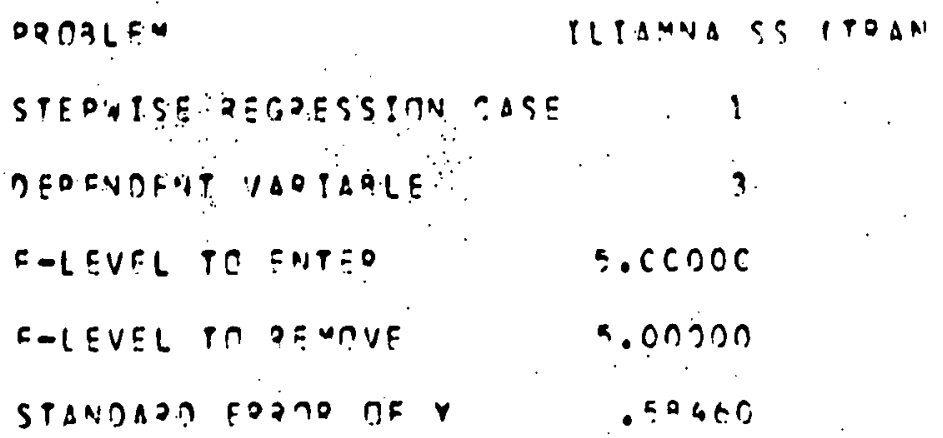


Table 11. Regression Analysis Results, Stream Sediment (cont lnued)

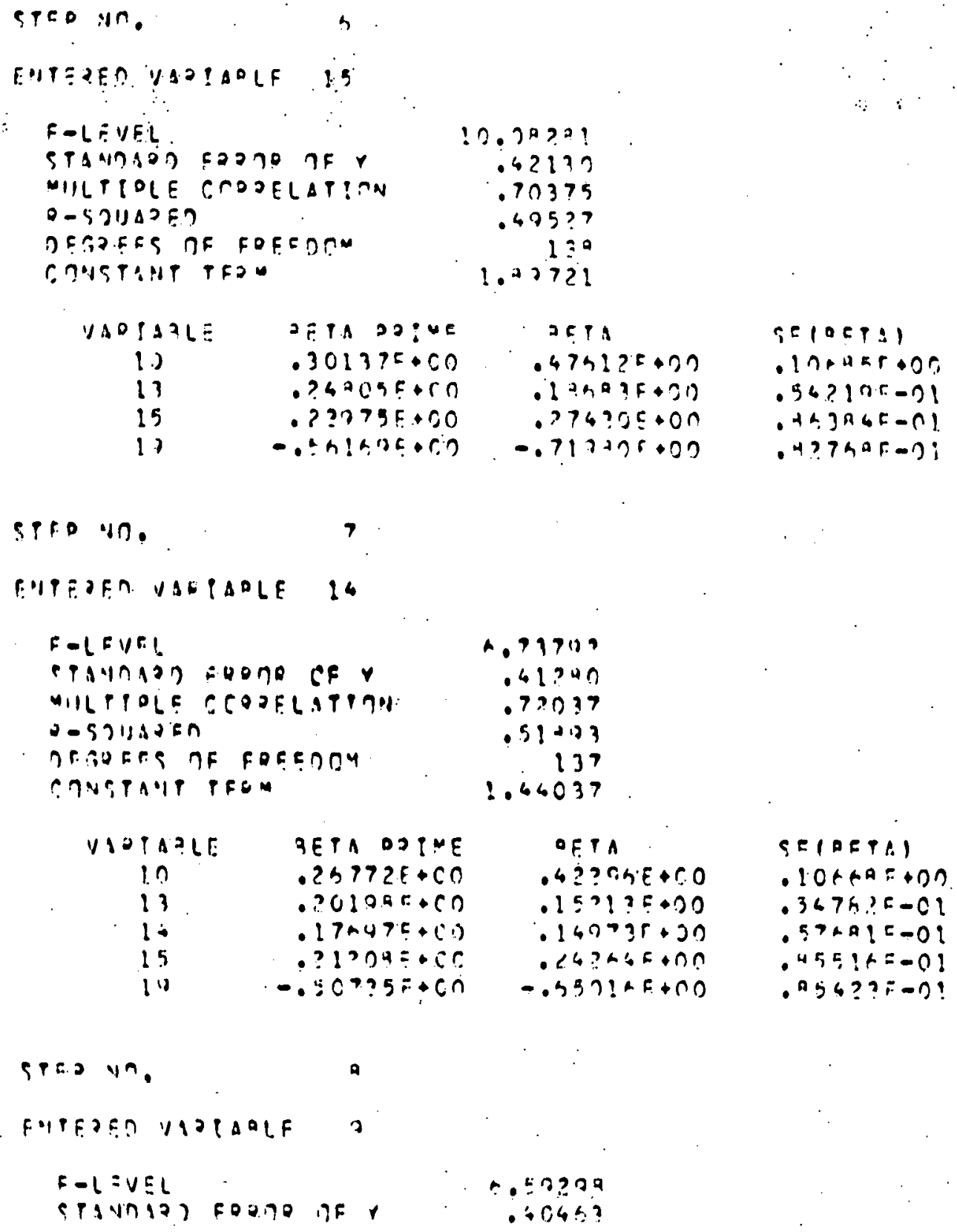


Table 11. Regression Analys1s Results, Stream Seuiment (continued)

MIJLTTOLE CORRELATICY

ว- 2014 ?

IFEZFFS TF FOEFCHM

Crastint TEQW
TF TIA: $D \supset T \div E$

$179065+C O$

$.279 ? 4 F+00$

:1:49? E+00

- 102 : $1 \mathrm{~F}+\mathrm{CD}$

- 2?.671E+00

- .607125+C0
.073564

.54117

134

1.3505?

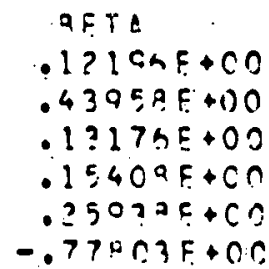

SE(RETA)

. $474095-01$

$.10477 \mathrm{E}+00$

. 5420 OE-01

- 545645-01

- $4407+5-01$

$.274 ? ? 5-01$

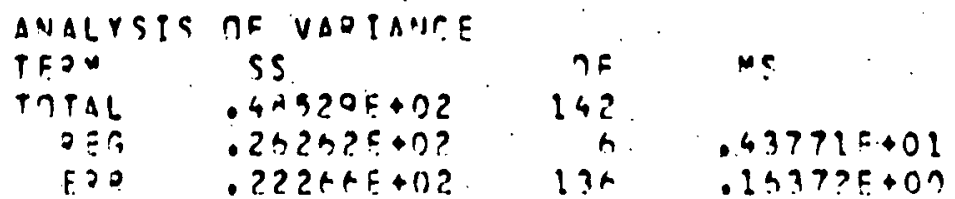


Table 12. Regresstori Analysi; 足:bults, lakes Sedlment

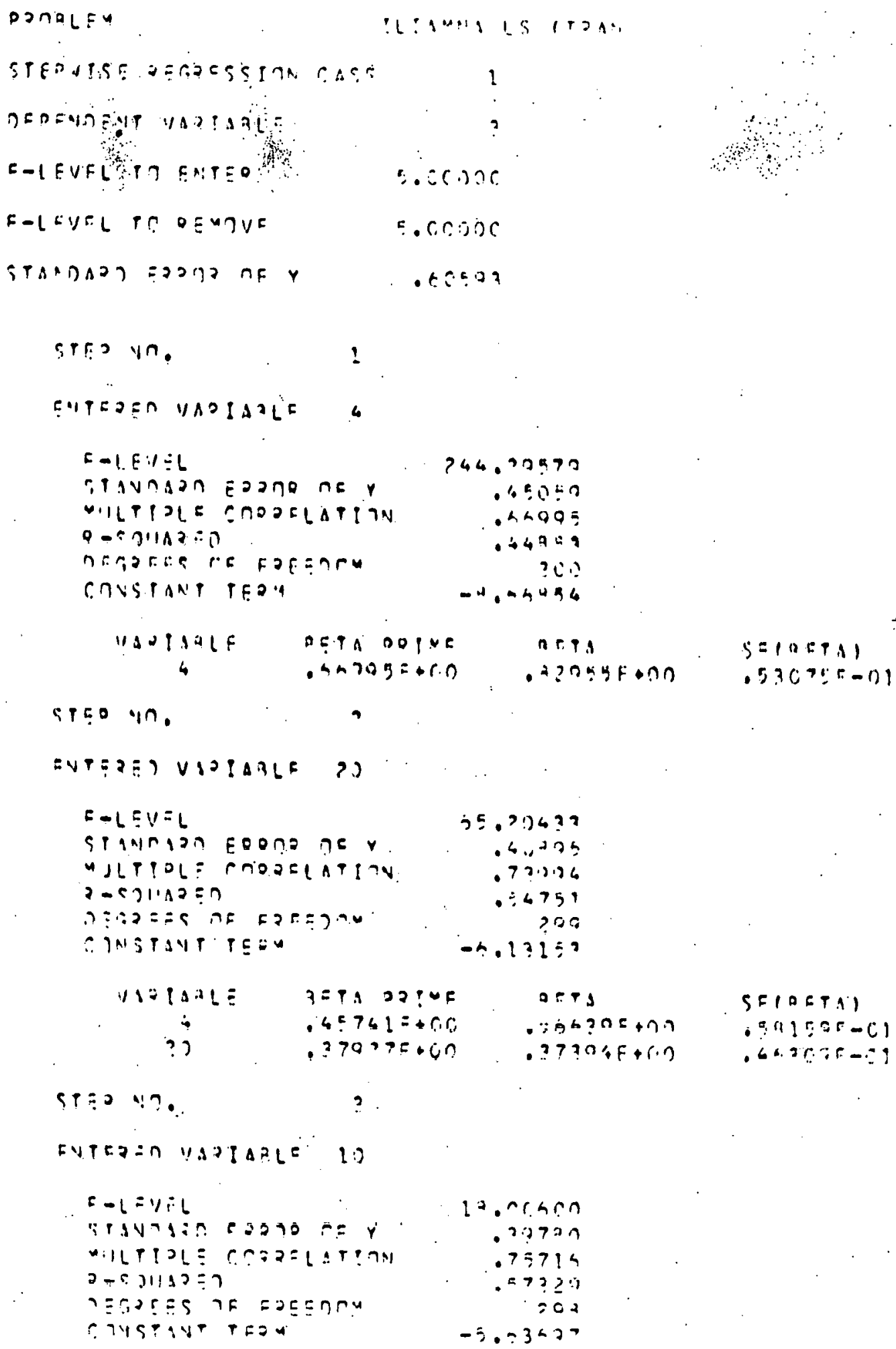


Table 12.. Regression inalysis Results, lake Sediment (continued)

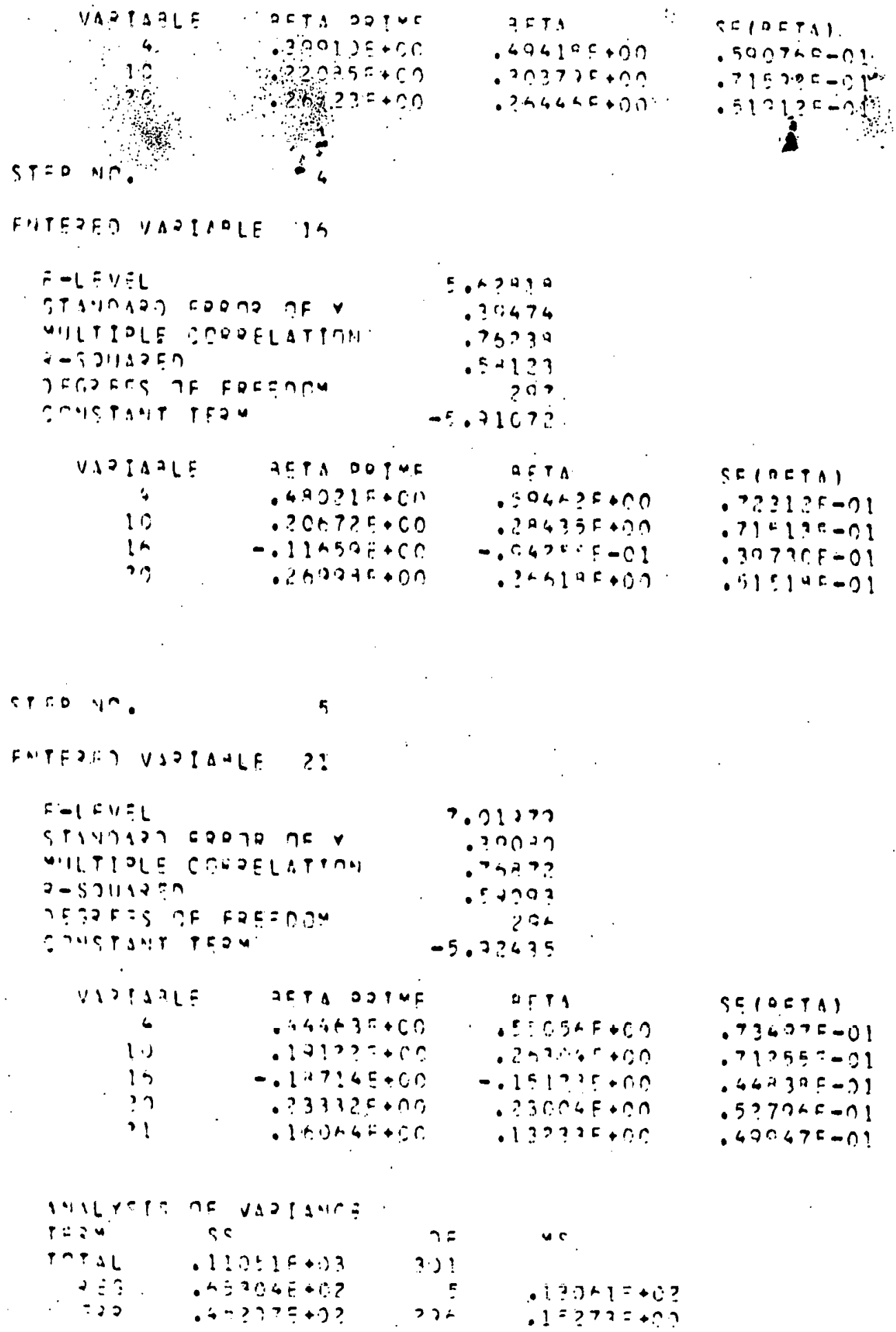

?.T. $.49410 c+00$ . ग0?735+0? ? $34445+101$

E.+? ? 90 . ? $1: 474$ $.75 ? 30$ $.5+123$ 20 ? $-5.710 ? ?$

\begin{tabular}{|c|c|}
\hline$V .1211^{2} 15$ & ZETA DOT FE \\
\hline it & $.490 ? 15 \cdot C 11$ \\
\hline $1 \%$ & ?CA $7 ? \mathrm{~F} \cdot \mathrm{CO}$ \\
\hline 19 & 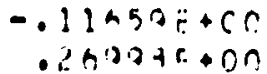 \\
\hline
\end{tabular}

SEIDETI

- 5007ar-n?.

- $7 ? 5 \sec e-1 ; j$ $.5121,25-26$

$$
\text { a }
$$$$
\text { -::CER F+C? }
$$$$
\text { - } z-3 \cdot \cdots r+c o
$$$$
\text { - is lour tin }
$$$$
\text { - isrng fon }
$$$$
\text { - } 13 ? \text { ? } 3 \text { +ror }
$$

SEIOEYA)

. 74075-01

$.71255=-01$

. 6 कर उ० $5-31$

. 5?.70م5-n! $.400475-0$ ? 
ILIAMNA, AK: STREAM SEDIMENT FACTOR SCORE 2

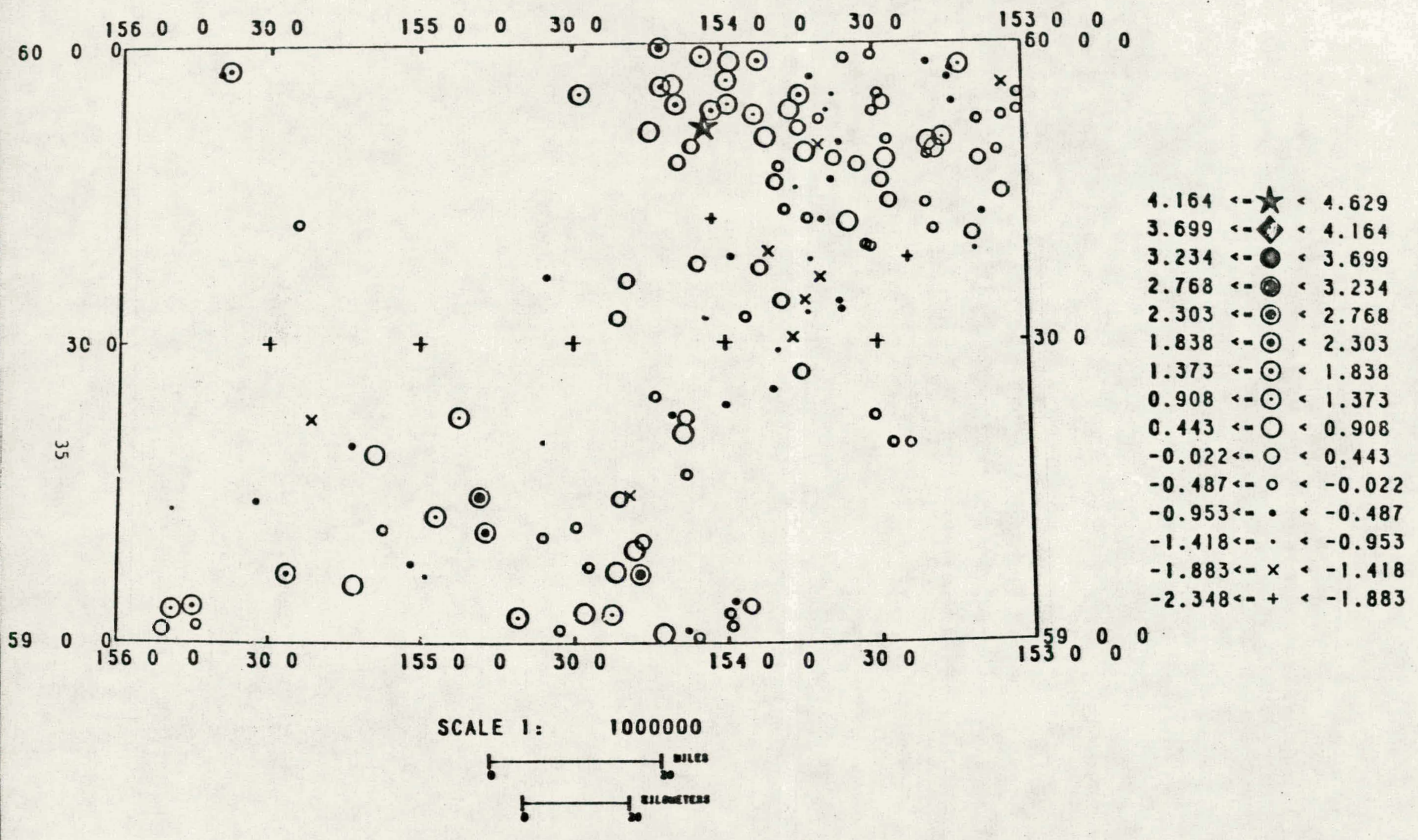

Figure 10. Stream Sediment Factor Score 2 
ILIAMNA, AY: STREAM SEDIMEIT RESIDUALS

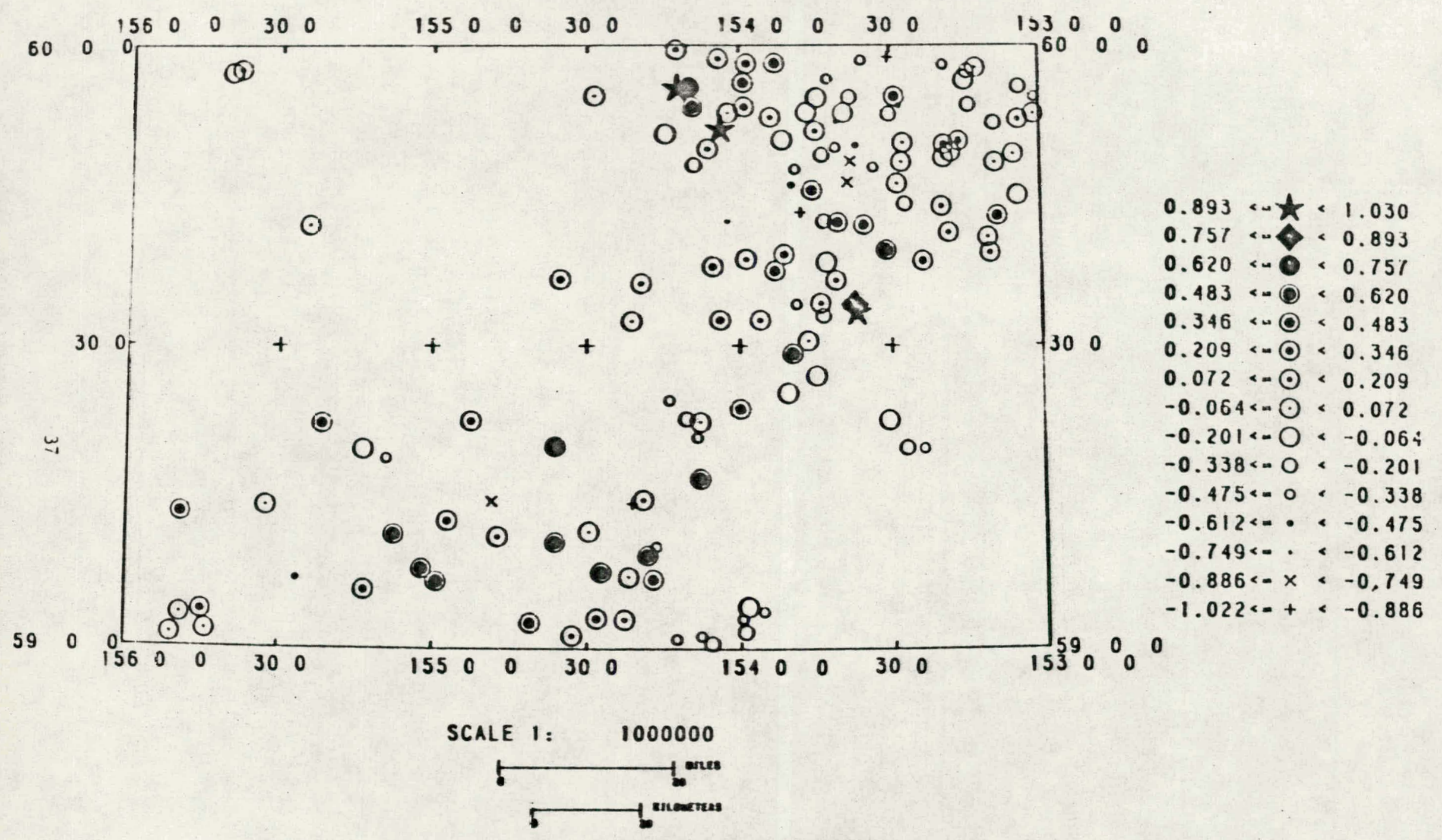

Figure 12. Stream Sediment Residuals 
IL IAMNA, AK: LAKE SEDIMENT FACTOR SCORE 2
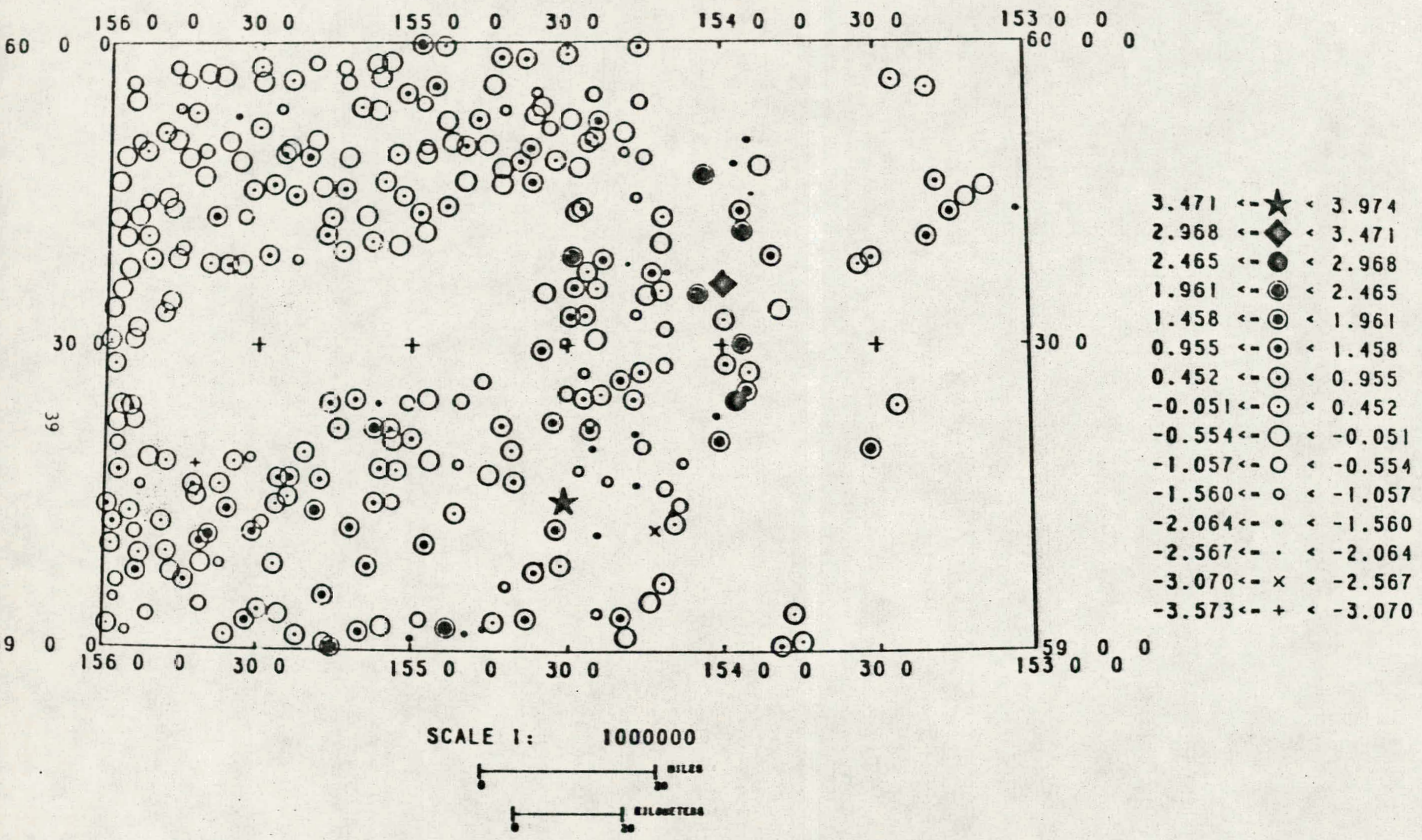

Figure 14. Lake Sediment Factor Score 2 
ILIAMNA, AK: LAKE SEDIMEHT FACTOR SCORE 3

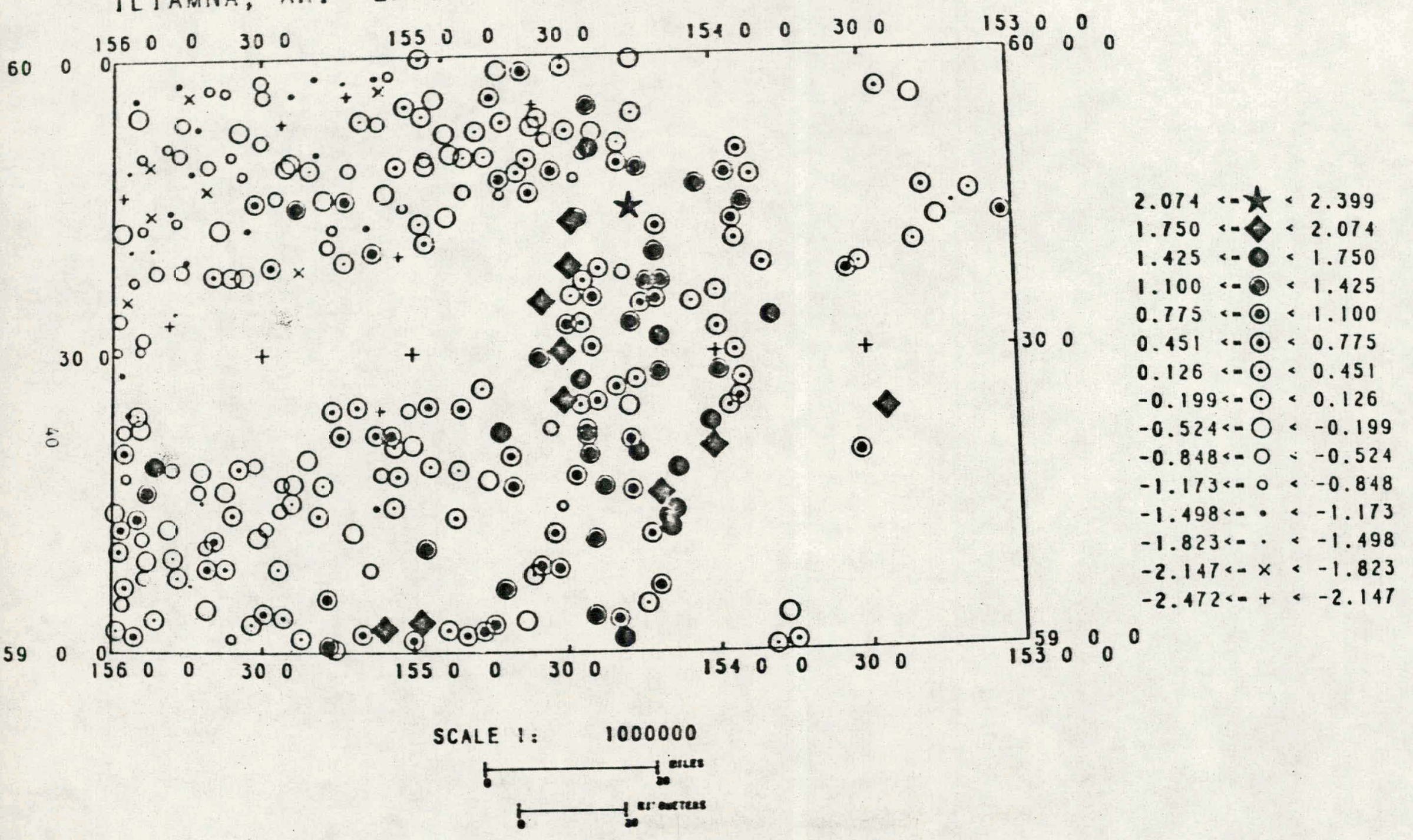

Figure 15. Lake Sediaent Factor Score 3 
ILIAMINA, AY: LAYE SEDIMEITT RESIDLILLS

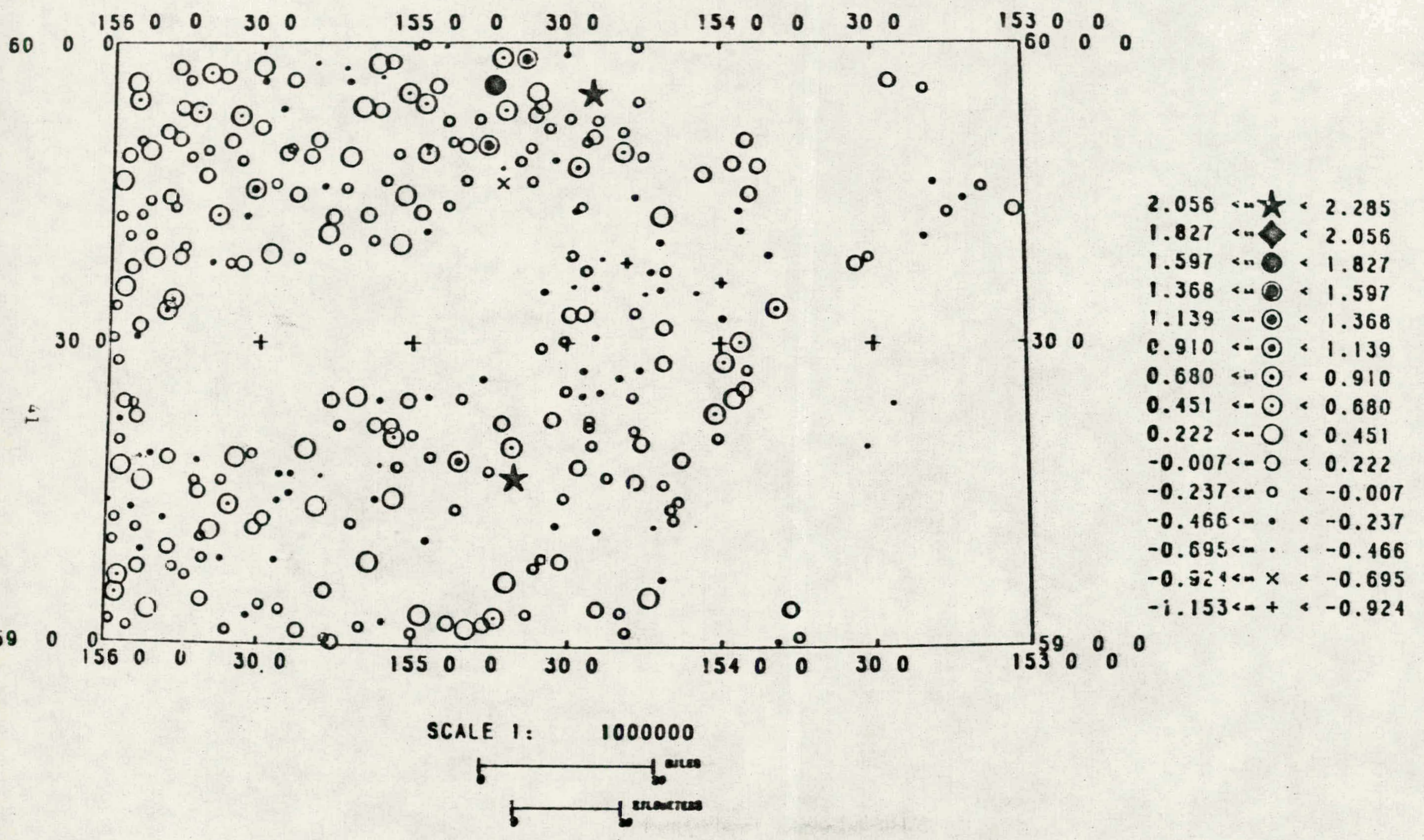

Figure 16. Lake Sediaent Residuals 
Planner, H. N., Apel, C. T., Fuka; H. A., George, W. E., Hansel, J. M., Hensluy, W. K., and Pircle, J., 1981, Uranlum Hydrogeochemlcal and stream sudtinent Keconnatagance Data Release for the Lendville NiMS Ruidrangle,. Culurilli, Including Concentraclons of forty-two dadtefonal ilements: U.S. Deprirtingnt of Energy Upen-File Report GJBX-13(81).

Shetcol, D. L., Jr., D'Andrea, R. F., Jr.. and zinkl, R. J.; 1980 , FACEDT: A rORTKAif Progran to Process Hydrogeochenlcal and Scraam Sedinent Keconnalsance Dica for Multivarlate Statistical Analysts: U.S. Department of Energy Upen-Flle Report CiJBX-246(80).

Ll.nk L, R. J., D'Andrea, R. F., Jr.; and Shettel, D. L., Jr., I98La, FoRTRAN Computer Programs to Read, Sort, and Ed le Loy Alamos Hydrogeochemlcal Laca, U.S. Department of Energy Open-Flle Repurt GJBX-11( 81$)$.

I'sib, Symbollc Plotting of Ceocheinlcal Exploration Data: U.S. Department of Enurgy Ipenatile Report (In preparation). 
Appentix a. Streas Selitert int

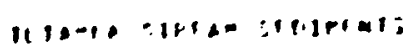

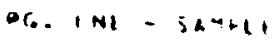

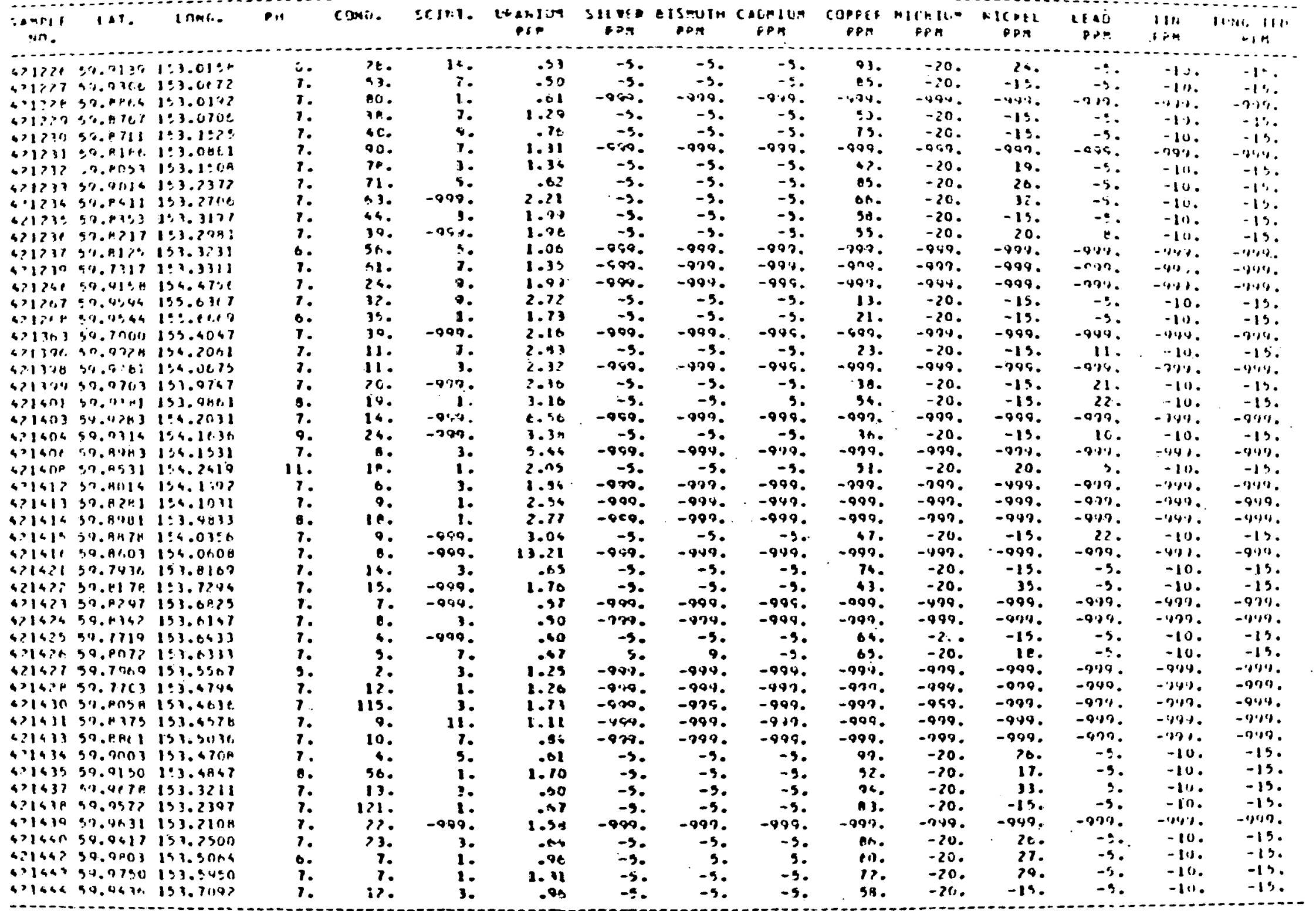




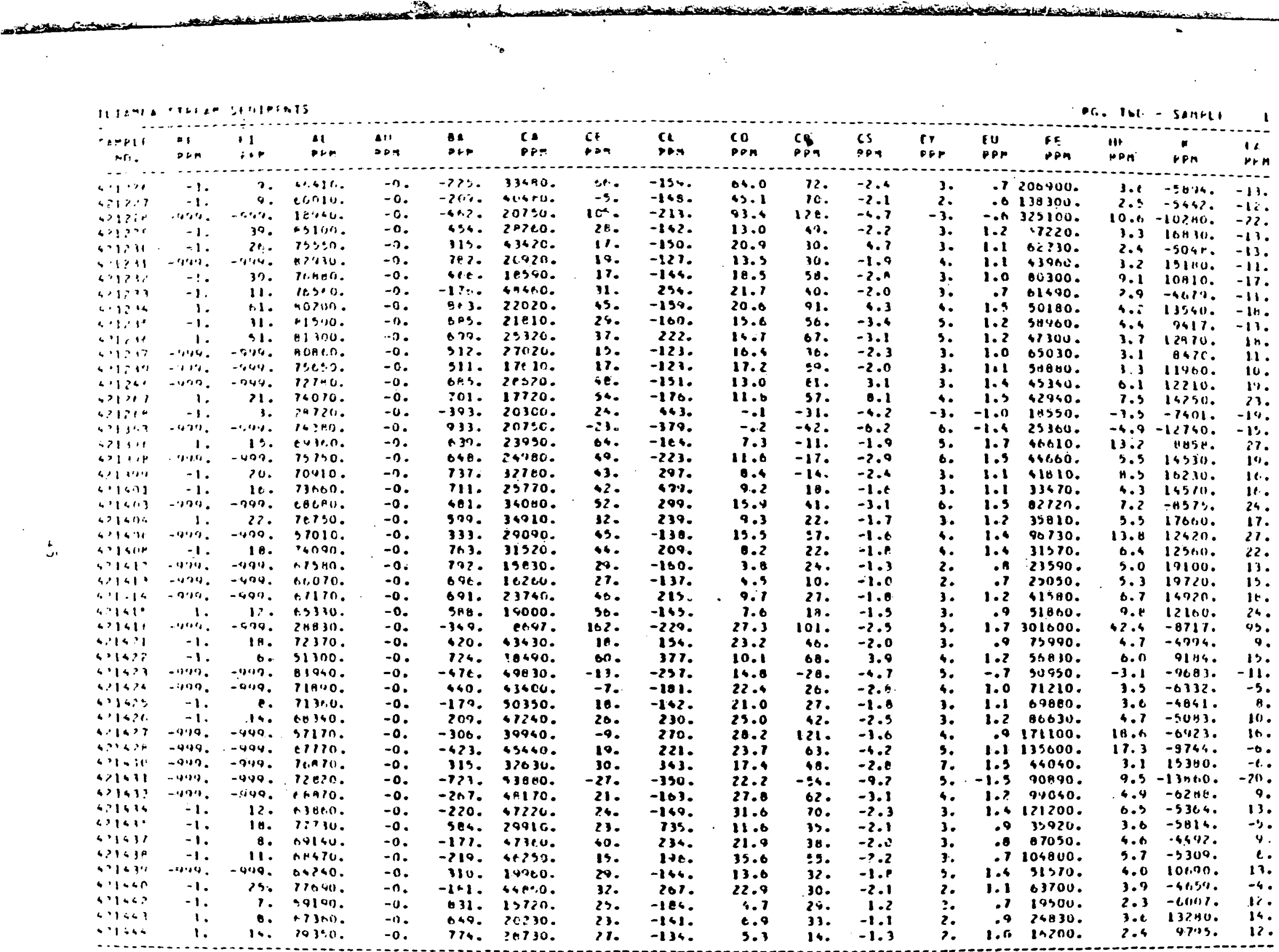


Heans stofan serirents

DC. nMt - janple 2

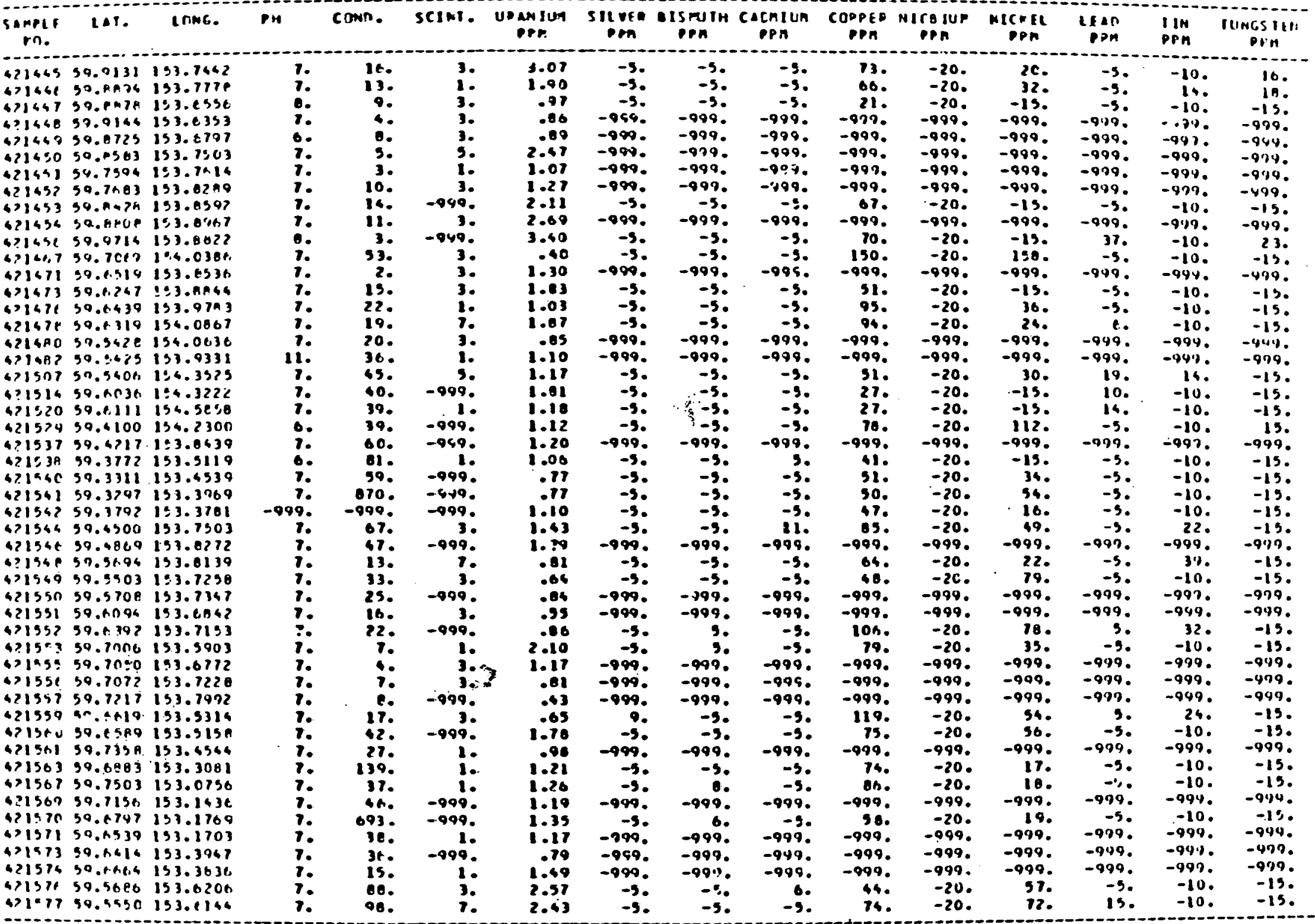




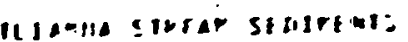

c. IUL - Salli

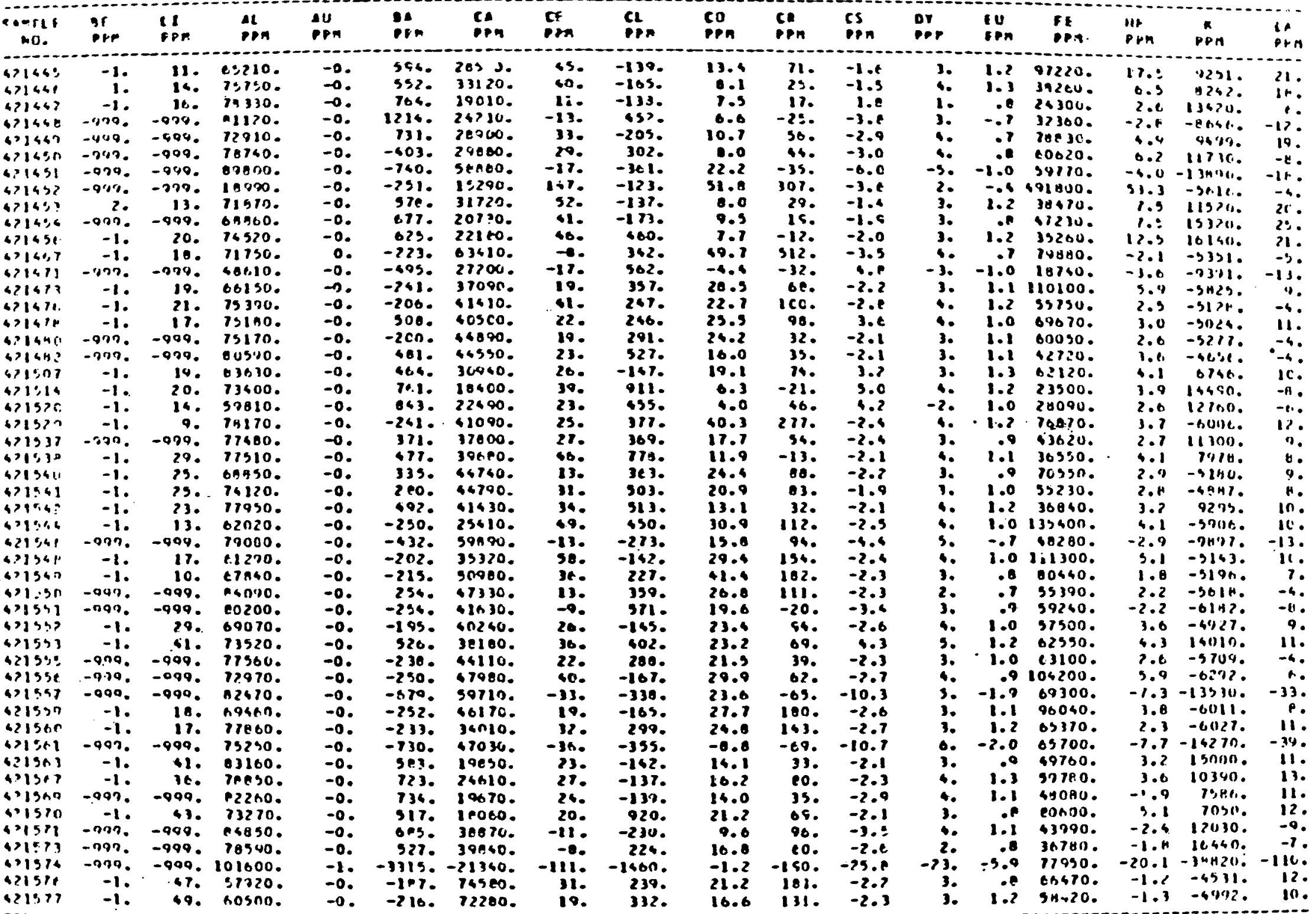


Hiene sioron strient:

>6. MHPfl - Sarte

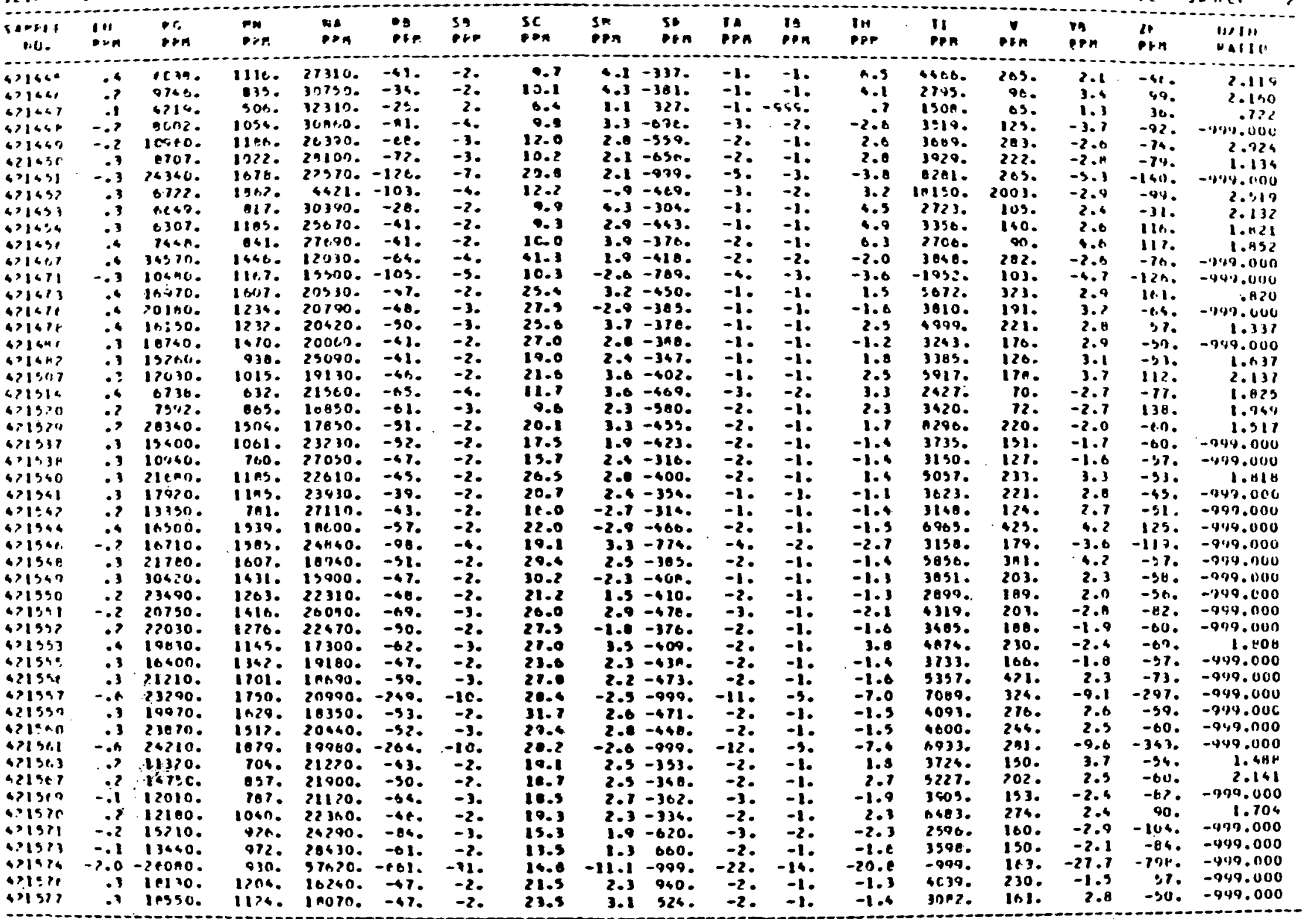


ll germa sporan stugher:

Pc. nite - SaYrt

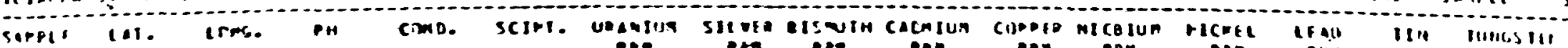

sis.

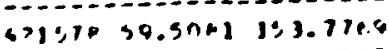

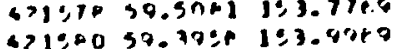

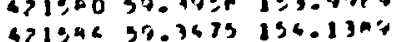
$4194039.347631: 4.1300$ 419.460 .39 .374 ? 194.1744 410.0230 .3331 19,4.enz2 $421907.37 .3336 \quad 19400072$ 4716350.1729193 .6401 $4211.2530 .1720,155.6480$ 6) $50.0 \mathrm{n} 22155.7644$ 411.77 59.ne22 195. 7645 4) 4.1617 30.2742123 .0700 47pint 5r.n433 1:6.0842

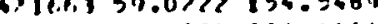

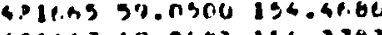
?llb7 39.06e? 134.3783

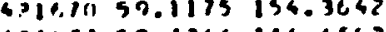
?lA 11 50.1716 136.6562 ?167P 59.1758 134.6033

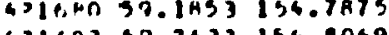
611 (A3 39.8633 154.0069 6164657.7266 154.7331 6716.7297 .170 .124 .6739 43162150.1221154 .4038 $418290.133 \mathrm{a} 154.301$ ? $417 n 0.97 .113$ e 134.2442 4170299.0161 134.2015 4170390.0214154 .1775 $6170459.0089134 .074 ?$ 42170539.0715 133.9HSA 61700 g0.06R4 133.906? 4171 ? 59.0 ntis 151.9264 4)11139.nend is 1.9161 41716 39.0.764 1:4.0133 4?1718 $30.1+19196.2739$ 4271150.7900134 .1204 $4117454.765 n 154.3150$ 421 1.25 59.2189154 .3509 $47171199.8106 \quad 156.9914$ 91119559.1114 134.9H67 42173659.1111795 .0362

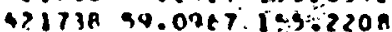
47176059.1161 153.641. $911742.54 .1122 \quad 15.5 .5553$ 47179350 :1AR9 199.1264 9175899.3130135 .1480 6179950.32801590280

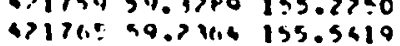

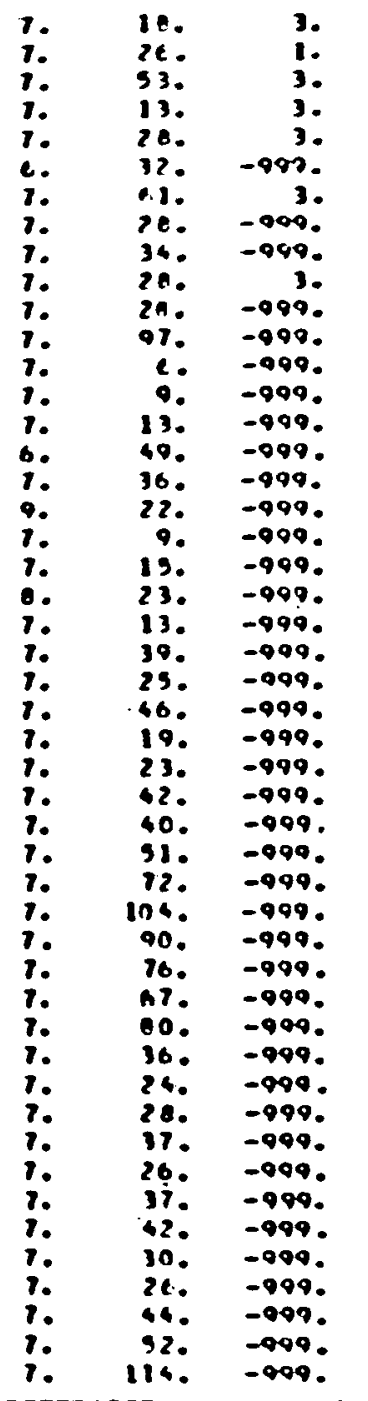

on om im ipn por Pn PPP ouy Iri: in

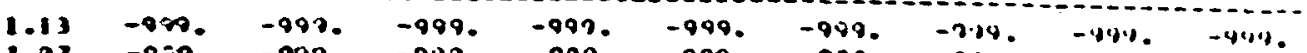

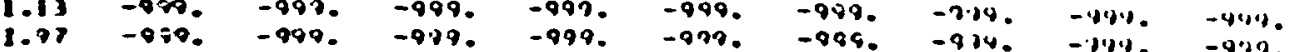

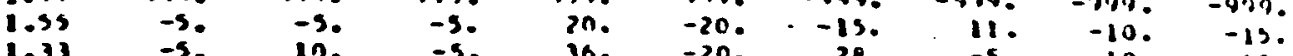

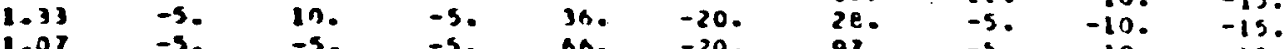

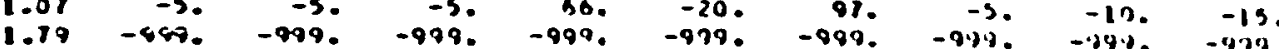

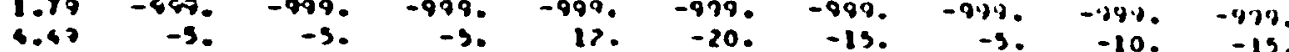

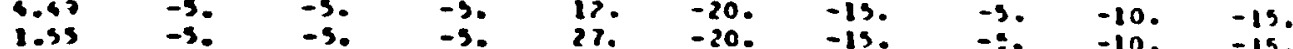

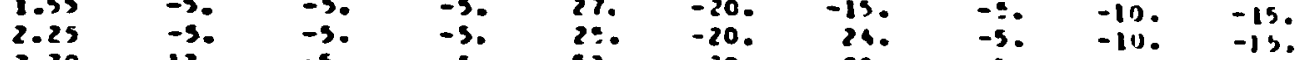

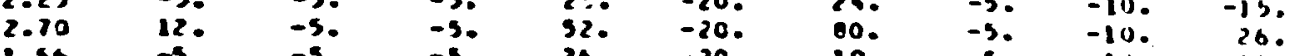

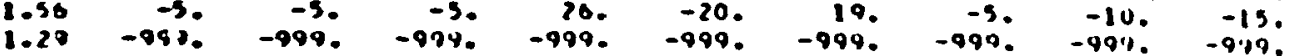

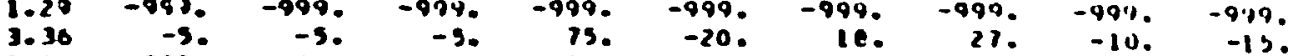

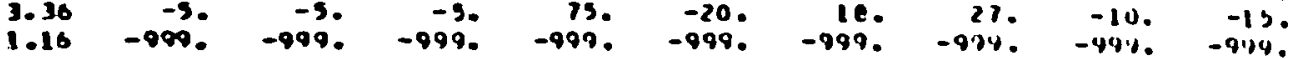

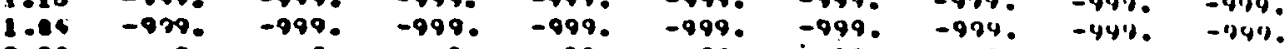

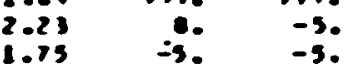
1. 2.21 - 999. 5.1350 .000$. $-990 .-990$. 1.06 - -989. 3.40 -979. -399. 2.02 -3. -3. $\rightarrow 99$ $1.10-900 .-999$. $1.91 \quad-649 . \quad-979$. $\begin{array}{lll}1.10 & -5 . & -5 . \\ .06 & -5 . & -5 .\end{array}$

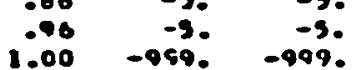

$.02 \quad-5 . \quad-5$.

1.19 $106-5$. $1.34 \quad-990$. 1.71 -990. -999. $\begin{array}{lll}1.05 & -5 . & -50 \\ .221 & -900 . & -999 .\end{array}$ $3.39-909$. 2.05 -090. -999. $\begin{array}{lll}2.00 & -50 & -5 . \\ 1.59 & -999 & -999\end{array}$ $1.55-0090$

2.05 -999. -999.

$1.48-990.099$

$1.29-99.59$.

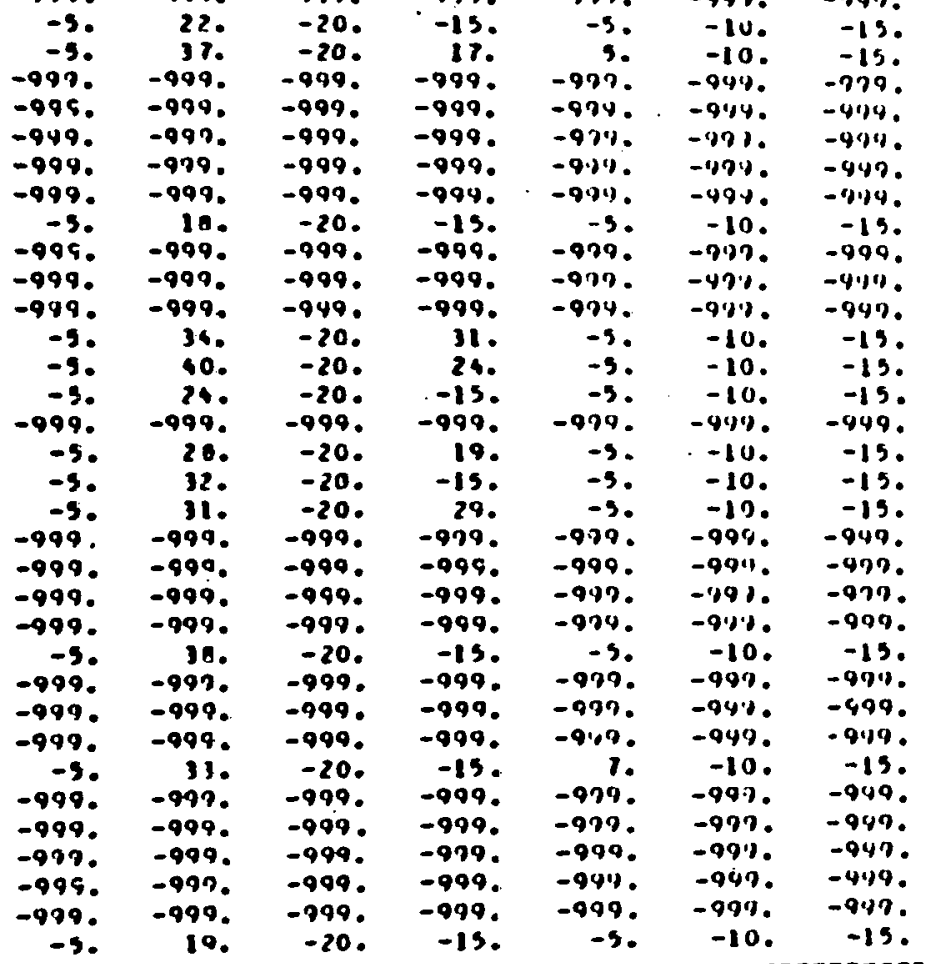


H1121 sibiat sinirfale

-G. IULI - SAMLLI

\begin{tabular}{|c|c|c|c|c|c|c|c|c|c|c|c|c|c|c|c|c|c|}
\hline $\begin{array}{l}\text { MPLI } \\
\text { Mo. }\end{array}$ & $\|_{P n}^{\prime \prime}$ & $\operatorname{lin}_{1}$ & al & All & ar & cen & CE & $\begin{array}{l}\text { CL } \\
\text { ren }\end{array}$ & $\begin{array}{l}C O \\
C P Y\end{array}$ & $\begin{array}{l}\text { ch } \\
\text { inn }\end{array}$ & $\begin{array}{l}\text { cs } \\
\text { ppn }\end{array}$ & $\begin{array}{l}n y \\
\text { ppn }\end{array}$ & $\begin{array}{l}\text { EU } \\
\text { PPn }\end{array}$ & IE & $\begin{array}{l}\text { IF } \\
\text { PPA }\end{array}$ & PPn & in \\
\hline 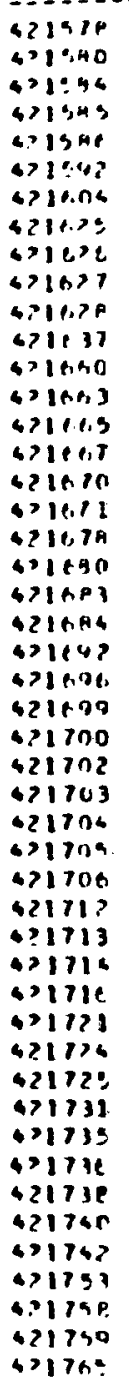 & 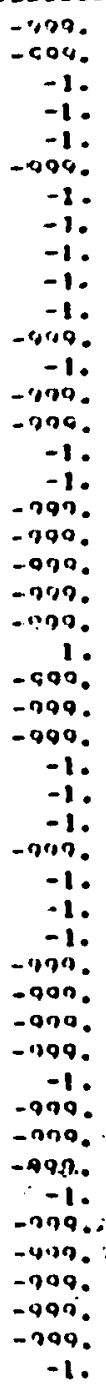 & $\begin{array}{r}-949 . \\
-979 . \\
11 . \\
31 . \\
21 . \\
-099 . \\
11 . \\
11 . \\
12 . \\
-11 . \\
12 . \\
-949 . \\
21 . \\
-949 . \\
-929 . \\
20 . \\
19 . \\
-999 . \\
-999 . \\
-979 . \\
-949 . \\
-999 . \\
20 . \\
-999 . \\
-999 . \\
-999 . \\
26 . \\
20 . \\
28 . \\
-999 . \\
25 . \\
43 . \\
430 \\
-499 . \\
-299 . \\
-999 . \\
-999 .\end{array}$ & 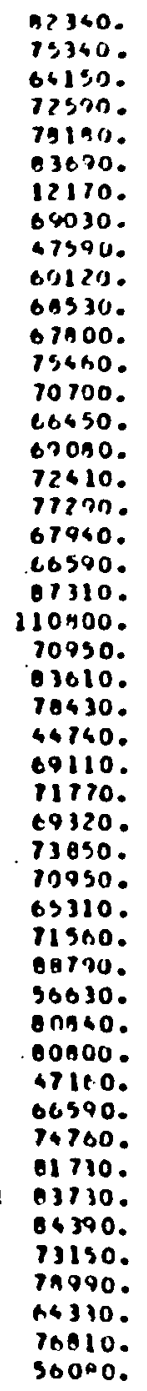 & $\begin{array}{l}-0 . \\
-0 . \\
-0 . \\
-1 . \\
-0 . \\
-0 . \\
-0 . \\
-0 . \\
-0 . \\
-0 . \\
-0 . \\
-0 . \\
-0 . \\
-0 . \\
-0 . \\
-0 . \\
-0 . \\
-0 . \\
0 . \\
-0 . \\
-1 . \\
-3 . \\
-0 . \\
-0 . \\
-0 . \\
-0 . \\
-0 . \\
-0 . \\
-0 . \\
-0 . \\
-0 . \\
-0 . \\
-0 . \\
-2 . \\
-0 . \\
-0 . \\
-0 . \\
-0 . \\
-0 . \\
-0 . \\
-0 . \\
-0 . \\
-1 . \\
-1.0 \\
-0 . \\
-0 . \\
-0 . \\
-0 .\end{array}$ & 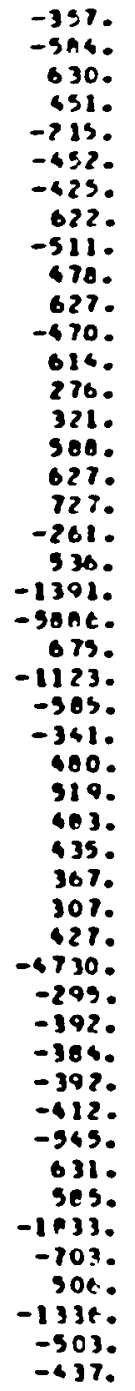 & 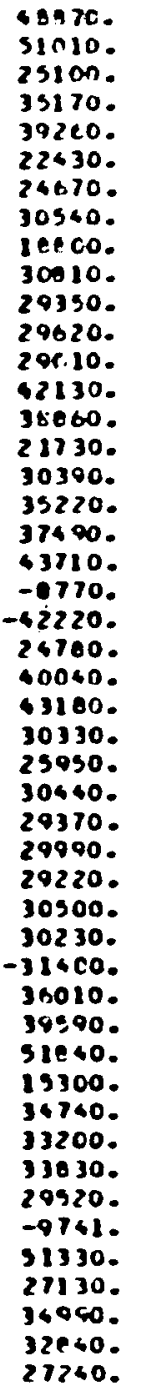 & 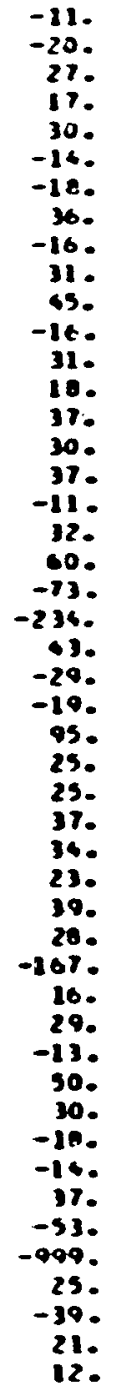 & 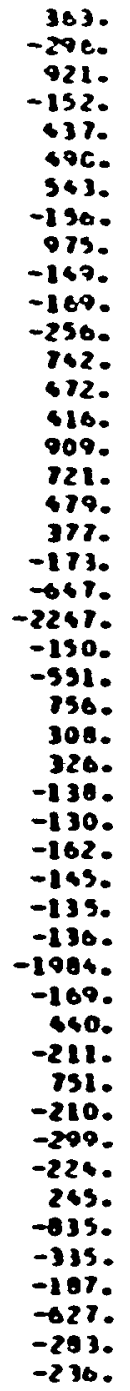 & 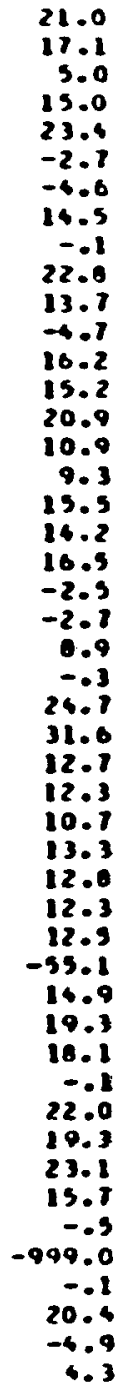 & 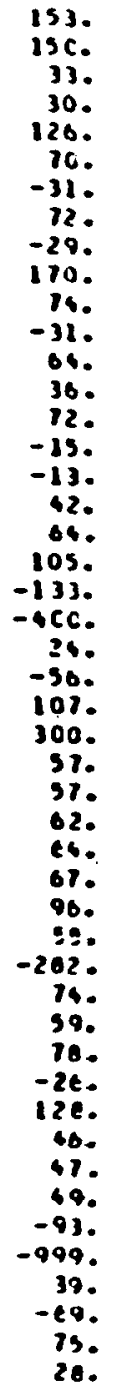 & 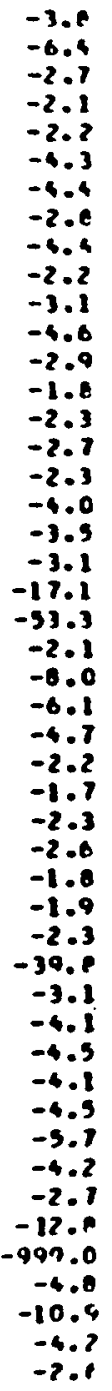 & 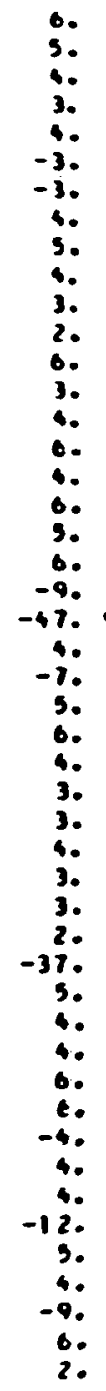 & 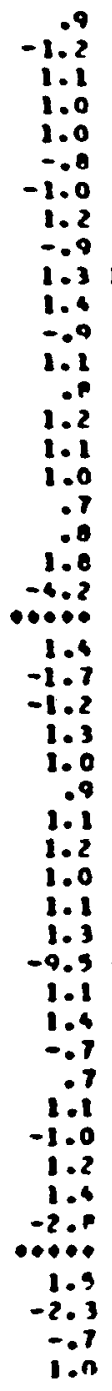 & 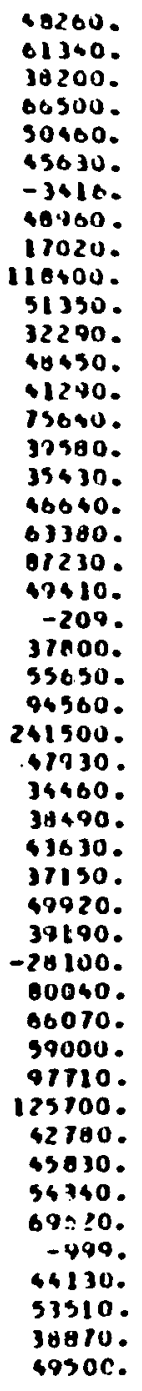 & 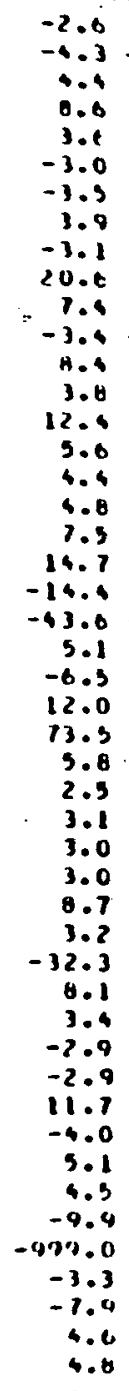 & 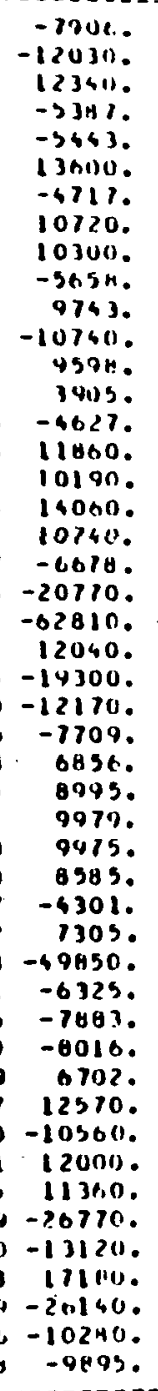 & 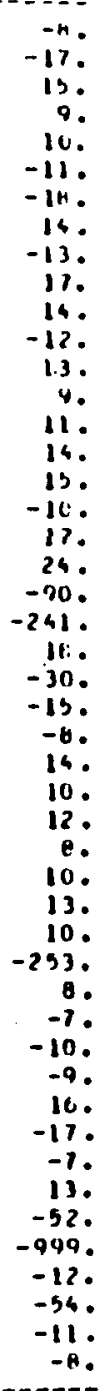 \\
\hline
\end{tabular}


He tapte ithea stolesnls

DC. Bllke - ja11L1

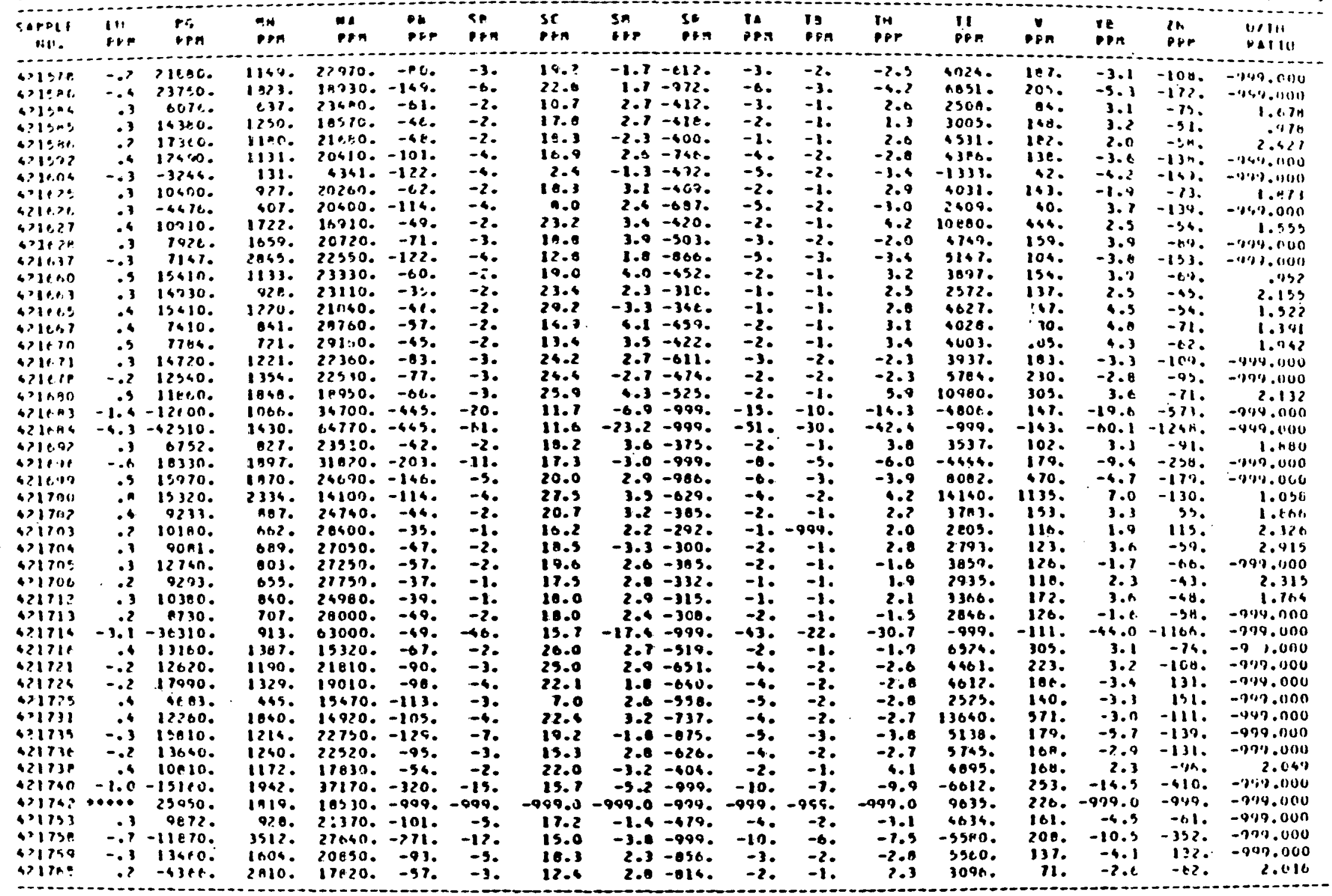




$$
-
$$


Appetilix B. Lake Salitent buta

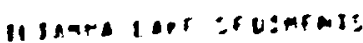

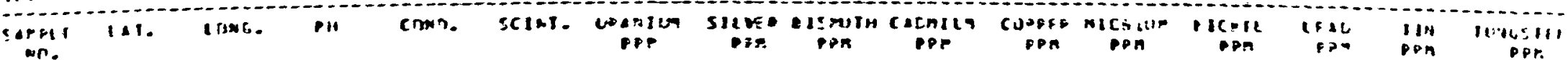

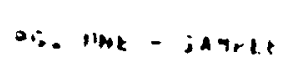

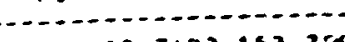

$41230=0.7192133 .7 \times 47$

4174030.7101 je6.8304

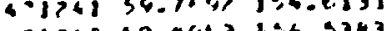

41748 30.4nis 3 136.5343

$471743 \% 9.7731$ J $26.642:$

471242 30.033 194.6311

$4176=50.7173$ 196.6580

$61747=7.7=00$ 1:4.6997

$47126459.0713134 .637 \%$

$41 ? 9094.0167194 .7117$

4123850.2746134 .0961

$4124399.9747194 .00,07$

$61734=4.07 .61193 .0122$

4 P1253 50.91 .90 155.1104

$41248=9.0424135 .10 \% 0$

$4125090.7390 \quad 159.2124$

6175009.9982153 .2264

$41790 \quad 0.919+135.3203$

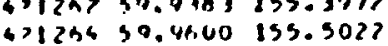

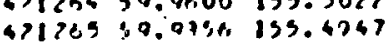

61798.59 .06310195 .6217

412 h⿱ 50.0497 193.671.4

42121059.931 .7 .135 .7430

4121190.9503 135.110e.

$41216511.1128 \quad 155.9242$

41773 :9.4nat 193.9164

A1Bir 50.4711 135.76?2

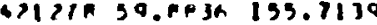

QIIIT 34.87H3 153.375e

417.157 .8542153 .5030

ज.174 90.4903139 .4311

G17N= 39.097 P 159.1603

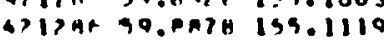

$412 n>50.9116$ 159.01日0

?1 ZA. 50.0?75 154.9267

47120059.9771194 .1372

47170250.7167 1:4.3779

$471243.90 .0 n 42$ 196. $5+11$

42179459.8575116 .5367

$4 \$ 1295: 9.0011154 .6316$

6123093.7217154 .7094

4120099.0207114 .7570

$4717.7959 .2767156 .01 A 3$

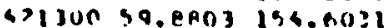

4'I3n? 99,nक31 1:4.41Us

$4 ? 130359.7706$ 154.077n

42170959.9347196 .0700

DI?CA 59.ATC3 1:4.HEB?

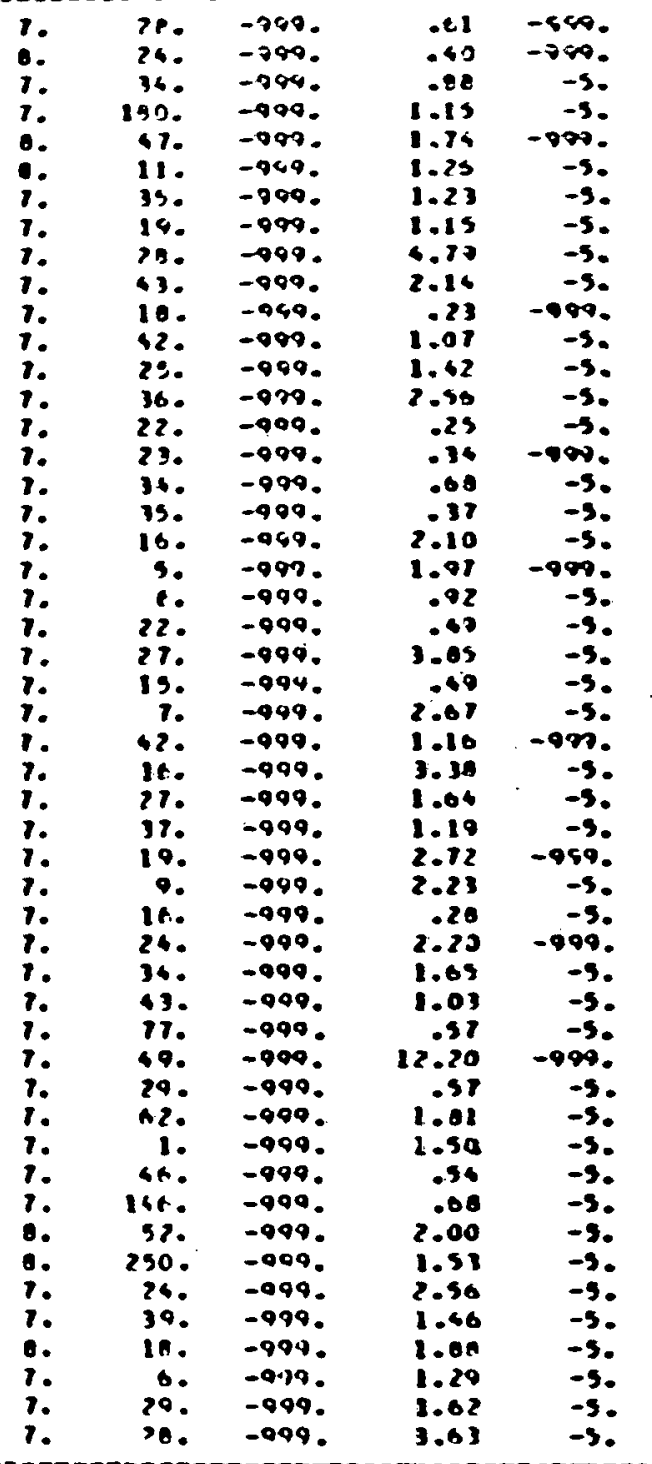

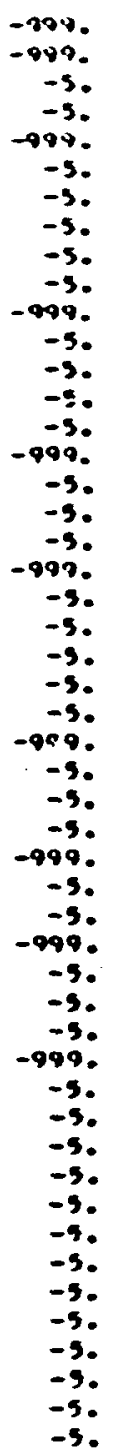

$-9.39$.

$-\infty 00$.

$-999$.

-

gan pon ppr.

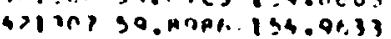




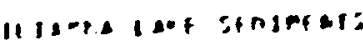

m.. Ine - sathe

:


$111,+1,1,2: 11,101+1=$ bi litede. iayel

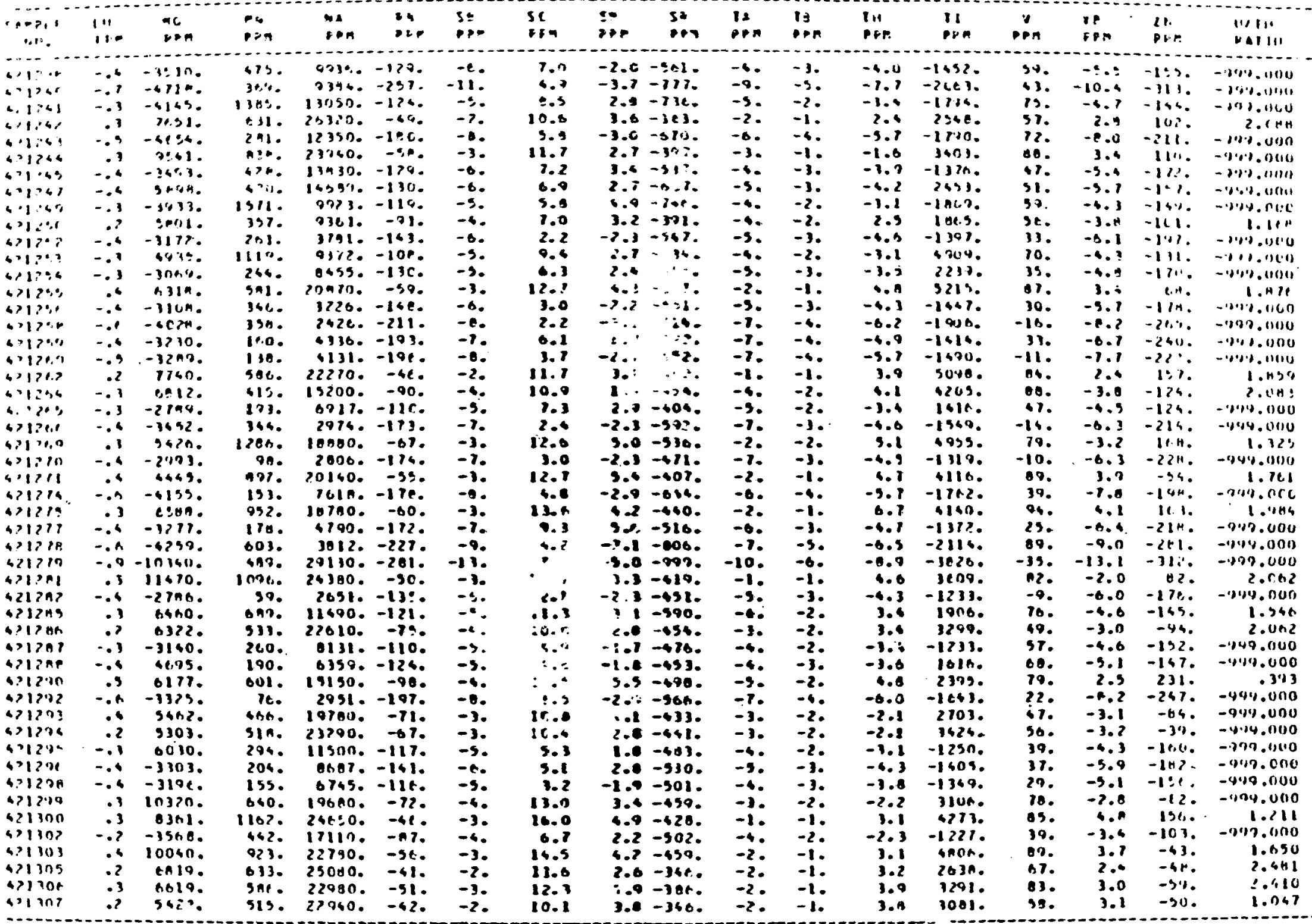




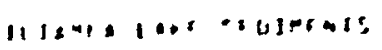
or. liat - solltt

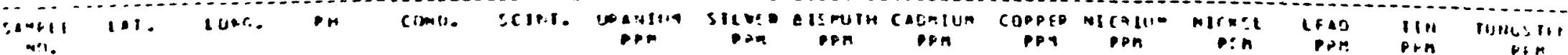
-

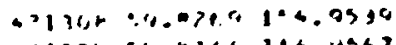

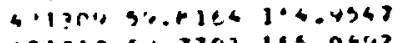
(i) -1111 30.1.3 19.00000 $41111^{\circ} 30 .+40713302094$

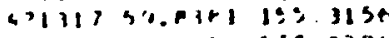
6111F $3 \% 0114$ 19.3304 $6131 \% 9 \%+2767133.4031$ $611>030.4104135 .6120$

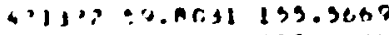

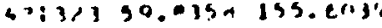
4I12: 90.4141 139.LA22 6137 5\%.Ante 1:3.736.1 แ1131 3\%.Ax.7 193.7172

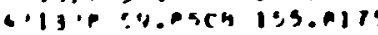
$41+140.10101$ 199.016 4111190.1333193 .4064 $41 ! 119 \% .+11+199.9412$ 1139890.71 .69 199.0150 4111190.1006199 .9602 $11116,9.6107155 .9378$ 4:111+ 9?.t.792 199.AOH 41711.90 .1119159 .0407 61117 91. 17t4 155.0122 4111090.1627193 .0067 1171094.1253 199.7467 6.134199 .1741159 .0461 $4.11363,56.712,15,5.0630$ $4314650.710^{4} 195.9496$ $4011450.75+1159.5206$ 41348909047 i49 9947 6100990.1472 is 950.1042

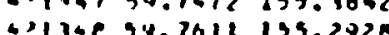
-1140 g0.7579 les.2200

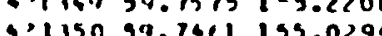

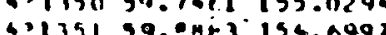

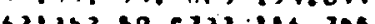
?i157 59.0294 194.0785 ?11939 50.P206 194.0256

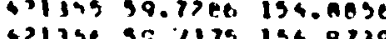
42115459.1125155 .1464 $? 1390,94,7091155.2030$

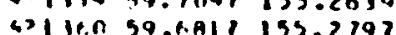

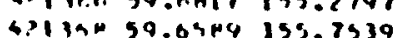
? 2131.059 .6419 199. 5097 61370 99.6uce 159.7697 4. 131190.036 1 95.9240

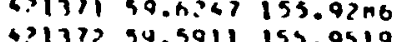
?21372 54.5011153 .9519 411639.3594195 .9741
4171350.3061135 .9967

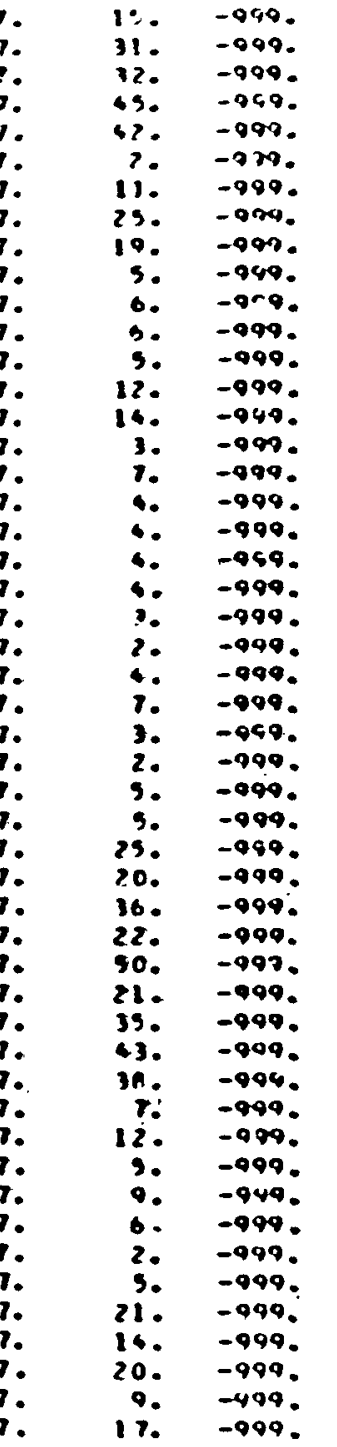

3.45

$\rightarrow$$$
-3 .
$$

13. -20

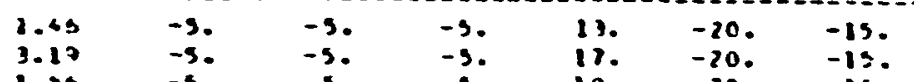
1.20

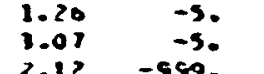
$1.73 \quad-9090$ $\begin{array}{ll}1.01 & -5 . \\ 1.22 & -5 .\end{array}$ $\begin{array}{ll}1.07 & -50 \\ 1.31 & -800 .\end{array}$ $\begin{array}{rrrr}1.37 & -050 & -999 . & -999 . \\ 1.87 & -5 . & -5 . & -5 . \\ 2.02 & -909 . & -999 . & -979 .\end{array}$ 1.61 -999. -999. -999. 2.16 - 50

1.08

3.53

$1.22-990$

1.98 -5.

3.08

2.17

2.18
1.59

$\begin{array}{ll}2.00 & -999 . \\ 1.00 & -909 .\end{array}$

i.es

1.79

1.60

1.06

-5.

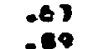

.69
.98
3.96

1.270 .90

$1.54 \quad-5$.

1.37

.09
.22

$2.22 \quad-5$.

$2.35 \quad-999$.

i.no

1.07 -5.

$2.09 \quad-5$.

$1.52 \quad-5$.

$1.72 \quad-5$.

1.33

$-5$.

-999.
-5.

-5.
-5.
-5

-5.

-79?.

-5.

-5.
-50

$-899$.

$-5$.

-995.

-998. -999.

-999
-5
-5

$-5$

$-3$

-999. -919.

$-3$.

$-3$

-999.
-5.
-5.

-999.
-5.

$-999$.

-999.
-3.

-999.
-5.

$-999 . \quad-999$.

10. -20. -13.

26. -20.

$-20$.

-15.
-15.

$\begin{array}{lll}-30 & -100 & -15 \\ -5 & -10 & -13 \\ 30 & -100 & -150\end{array}$

-15.
-909.

$\begin{array}{lll}\text { 5. } & -100 & -13 . \\ 11 . & -100 & -13 . \\ 11 . & -10 . & -1 \%\end{array}$

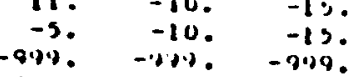

999. -999. -999.

35. $-20 . \quad-15$.

29.

$-999$.

-20.
-999.

$-20$.

-15.
-999.

$-15$.

$-909$.

$-949$.

$\begin{array}{rrr}-999 . & -999 . & -949 . \\ 11 . & -20 . & -13 . \\ 29 . & -20 . & -15\end{array}$

$\begin{array}{lll}29 . & -20 . & -15 . \\ 29 . & -20 . & 2 \mathrm{C} .\end{array}$

33.
-999.

$-999$.

$-999$.

999.

24.

20.
-909.

-20.
-20.

-20.
-899.

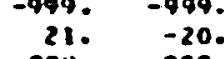

$-994$.

$-999 . \quad-999$.

$-999 . \quad-399$.

$\begin{array}{ll}22 . & -20 . \\ 27 . & -20 .\end{array}$

22.

$-999$.

$-999$.

23.

-999.
24.

-997.
18.

39.

3.

$-5$.

$-5$.

$-5$.

$-5$.

$-5$.

29.

-20.
-999.

26.

31.

16. -20 .

11. -20 .

-20.
-20.

-15.
-999.

$-999$.

15
-999

$-999$.

-999.
-19.
23.

23.
-15.

-15.
-999.

$\begin{array}{ll}-999 . & -999 . \\ -999 . & -999 .\end{array}$

-999.
-25.

$-15$.

$-12$.

-999
-099
-98

-999.
-15.

-15.
20.

-949.
-15.

-15.
15.

-15.
-15

-15.
-13.

-13.
-150

-13.
-15.

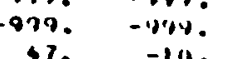

$\begin{array}{lll}\text { 7. } & -10 . & -15 . \\ -5 . & -10 . & -13 .\end{array}$

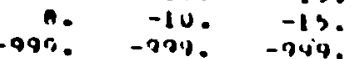

$\begin{array}{rrr}-3 . & -10 . & -15 . \\ -909 & -949 & -999\end{array}$

$-999 .-947 . \quad-979$.

$-949 . \quad-949 . \quad-944$.

$\begin{array}{lll}3 . & -10 . & -13 . \\ -3 . & -10 . & -15\end{array}$

$\begin{array}{rrr}5 . & -13 . & -13 . \\ -999 & -49 . & -13 .\end{array}$

1. - - $10.10-919$.

$\begin{array}{lll}-5 . & -10 . & -15 . \\ -5 & -10 . & -15\end{array}$

-999. -99.1 -994.

$\begin{array}{lll}-999 . & -998 . & -999 .\end{array}$

$-909 . \quad-99.1 . \quad-979$.

$\begin{array}{lll}-999 . & -941 . & -9114 .\end{array}$

-3. -10. -15.

-5. -10 - -15.

$-979 . \quad-994 . \quad-999$.

$-5 . \quad-10 .-15$.

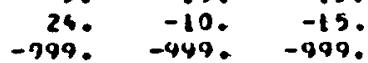

-3. -10. - 15.

-5. -10. -13.

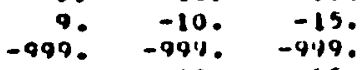

6. $-10 . \quad-15$.

10. -10. -15.

-3. -10. - -15.

$\begin{array}{lll}-5 . & -10 . & -15 . \\ -5 & -100 & -15\end{array}$

$\begin{array}{lll}-5 . & -100 & -15 \\ -5 . & -10 . & -19 .\end{array}$

10
80


Hianse cane sroimints

Pr. IIIGEF - sanre

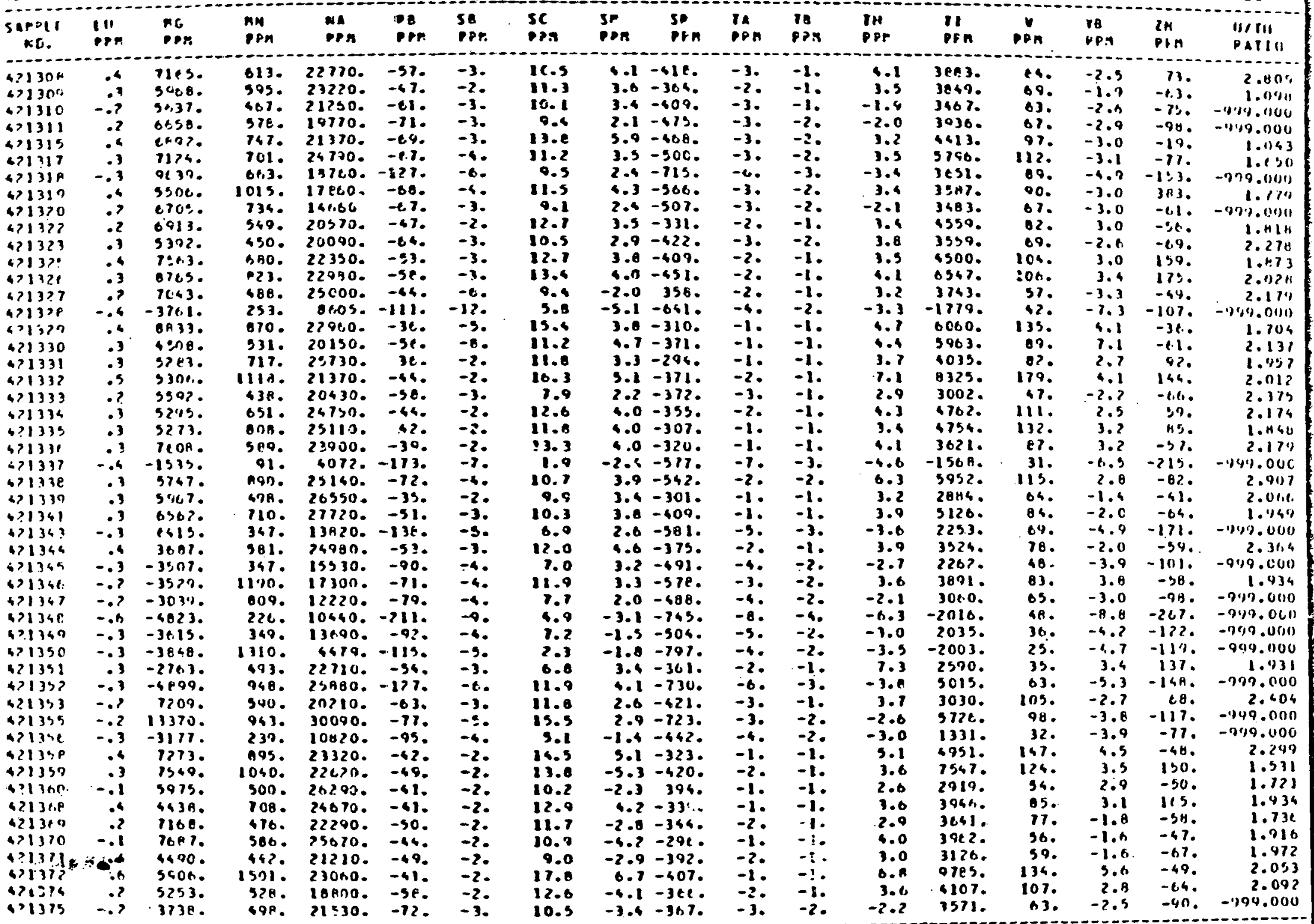


PC. CEI - SAMIIE,

\begin{tabular}{|c|c|c|c|c|c|c|c|c|c|c|c|c|c|c|c|}
\hline $\begin{array}{l}\text { ate } \\
\text { *n. }\end{array}$ & 107. & InNf.. & 11 & (1) & בו & loglen & $\begin{array}{c}\text { lited } \\
\text { Dras }\end{array}$ & $\begin{array}{l}\text { ismush } \\
\text { ips }\end{array}$ & $\begin{array}{l}\text { Donsut } \\
\text { ip. }\end{array}$ & $\begin{array}{l}\text { oppef } \\
\text { oph }\end{array}$ & $\begin{array}{c}\text { nlog } 11 . n \\
\text { if }\end{array}$ & $\begin{array}{c}\text { ICade } \\
\text { oph }\end{array}$ & $\begin{array}{r}\text { IPU } \\
\text { PPA }\end{array}$ & $\begin{array}{l}\text { IIN } \\
\text { Irn }\end{array}$ & $\begin{array}{c}\text { Iric }=\text { Ith } \\
\text { pin }\end{array}$ \\
\hline 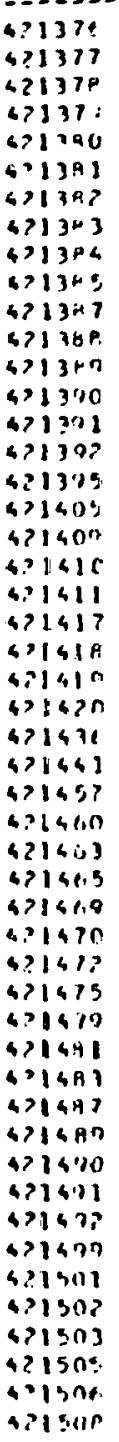 & 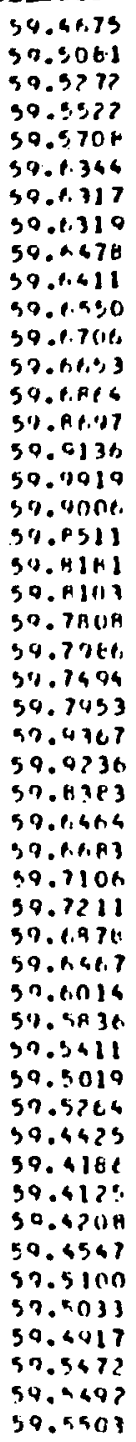 & 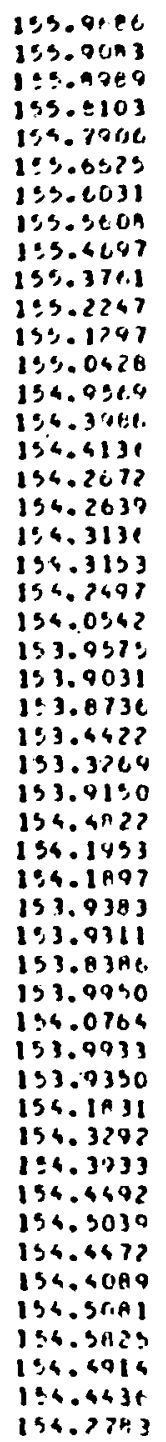 & 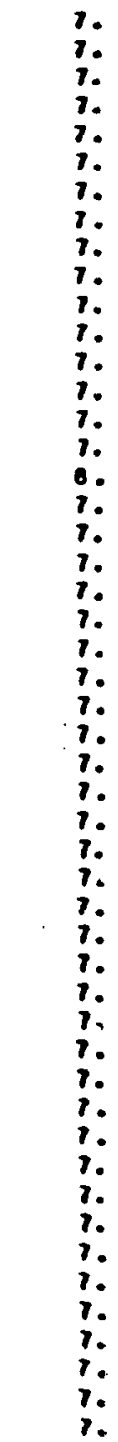 & 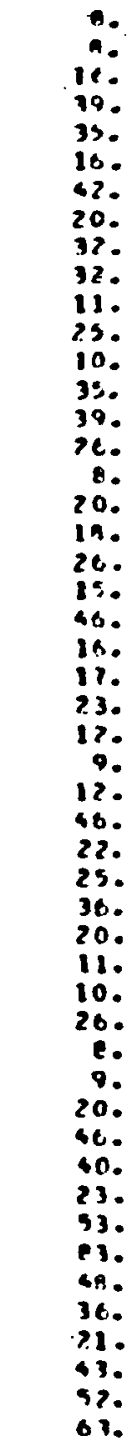 & $\begin{array}{l}-949 . \\
-909 . \\
-949 . \\
-649 . \\
-909 . \\
-998 . \\
-999 . \\
-999 . \\
-999 . \\
-999 . \\
-999 . \\
-999 . \\
-999 . \\
-999 . \\
-999 . \\
-990 . \\
-990 . \\
-999 . \\
-999 . \\
-999 . \\
-099 . \\
-999 . \\
-999 . \\
-999 . \\
-999 . \\
-999 . \\
-999 . \\
-999 . \\
-999 . \\
-999 . \\
-999 . \\
-999 . \\
-999 . \\
-999 . \\
-999 . \\
-999 . \\
-999 . \\
-999 . \\
-990 . \\
-999 . \\
-999 . \\
-999 . \\
-999 . \\
-999 . \\
-990 . \\
-999 . \\
-999 . \\
-909 . \\
-999 . \\
-990 .\end{array}$ & 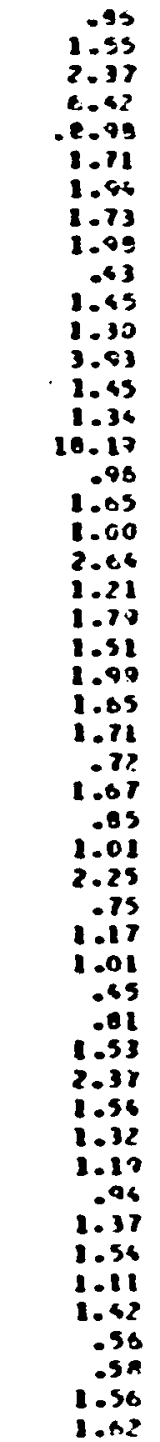 & 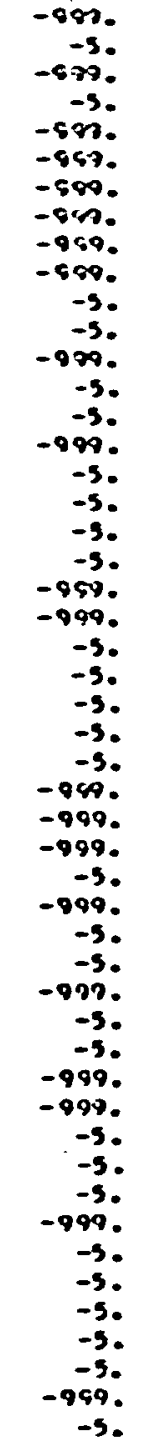 & 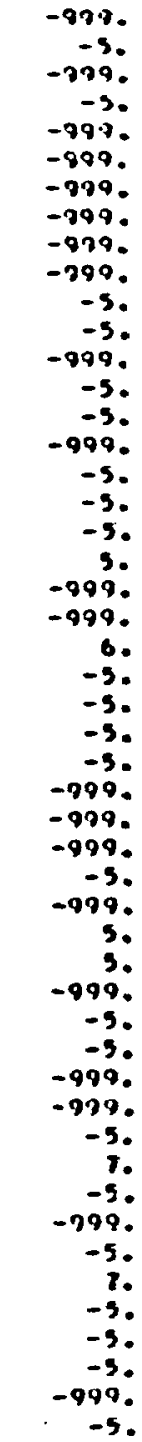 & $\begin{array}{r}-999 . \\
-5 . \\
-920 . \\
-5 . \\
-999 . \\
-979 . \\
-999 . \\
-995 . \\
-999 . \\
-969 . \\
-5 . \\
-5 . \\
-999 . \\
-5 . \\
-5 . \\
-999 . \\
-5 . \\
-5 . \\
-5 . \\
-3 . \\
-999 . \\
-999 . \\
-5 . \\
-5 . \\
-5 . \\
-5 . \\
-5 . \\
-999 . \\
-9.19 . \\
-999 . \\
-5 . \\
-909 . \\
-5 . \\
-5 . \\
-999 . \\
-5 . \\
-5 . \\
-999 . \\
-999 . \\
-5 . \\
-5 . \\
-5 . \\
-999 . \\
-5 .\end{array}$ & $\begin{array}{r}-979 . \\
25 . \\
-999 . \\
33 . \\
-997 . \\
-959 . \\
-994 . \\
-999 . \\
-999 . \\
-999 . \\
26 . \\
30 . \\
-999 . \\
33 . \\
16 . \\
-999 . \\
32 . \\
21 . \\
29 . \\
24 . \\
-994 . \\
-999 . \\
20 . \\
31 . \\
40 . \\
30 . \\
25 . \\
-929 . \\
-999 . \\
-999 . \\
-10 . \\
-991 . \\
34 . \\
33 . \\
-999 . \\
26 . \\
40 . \\
-999 . \\
-999 . \\
31 . \\
37 . \\
41 . \\
-999 . \\
15 . \\
24 . \\
23 . \\
28 . \\
17 . \\
-999 . \\
21 .\end{array}$ & $\begin{array}{r}-999 . \\
-20 . \\
-999 . \\
-20 . \\
-999 . \\
-299 . \\
-290 . \\
-999 . \\
-999 . \\
-999 . \\
-20 . \\
-20 . \\
-999 . \\
-20 . \\
-20 . \\
-999 . \\
-20 . \\
-20 . \\
-20 . \\
-20 . \\
-999 . \\
-999 . \\
-20 . \\
-20 . \\
-20 . \\
-20 . \\
-20 . \\
-999 . \\
-997 . \\
-999 . \\
-20 . \\
-999 . \\
-20 . \\
-20 . \\
-294 . \\
-20 . \\
-20 . \\
-999 . \\
-999 . \\
-20 . \\
-20 . \\
-20 . \\
-999 . \\
-20 . \\
-20 . \\
-20 . \\
-20 . \\
-20 . \\
-997 . \\
-20 .\end{array}$ & 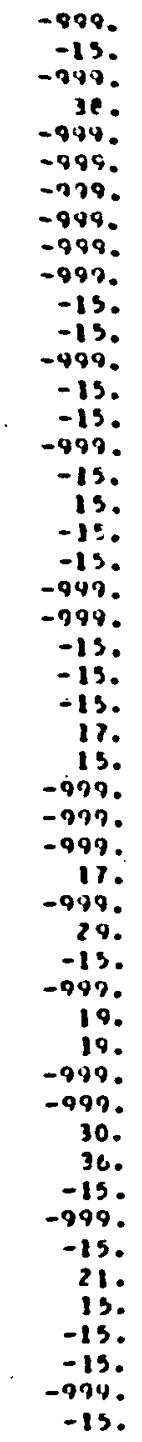 & 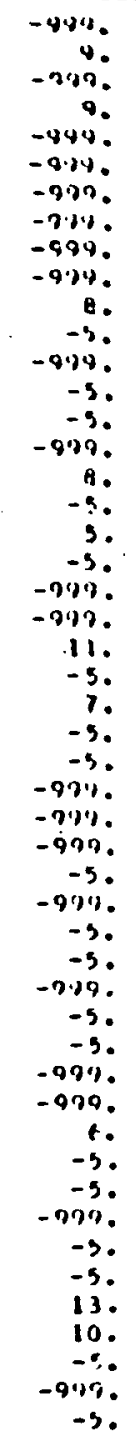 & 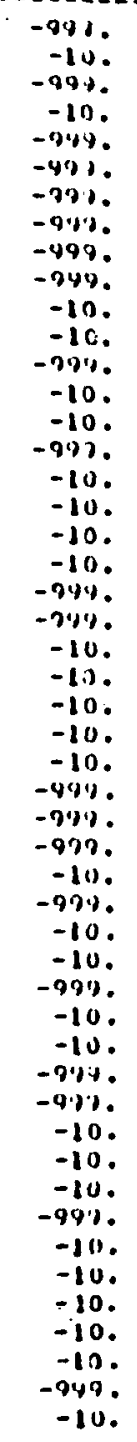 & 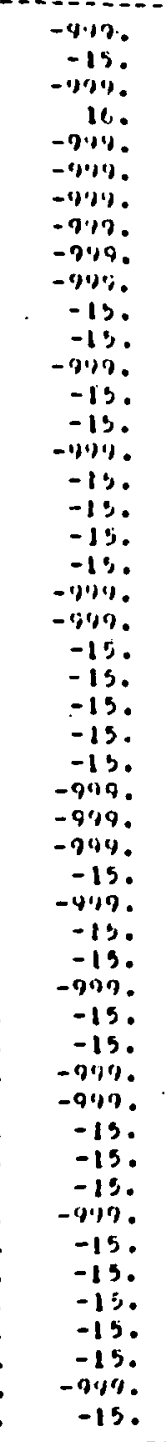 \\
\hline
\end{tabular}




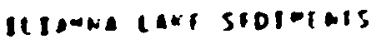

-G. IVI - SAMLA

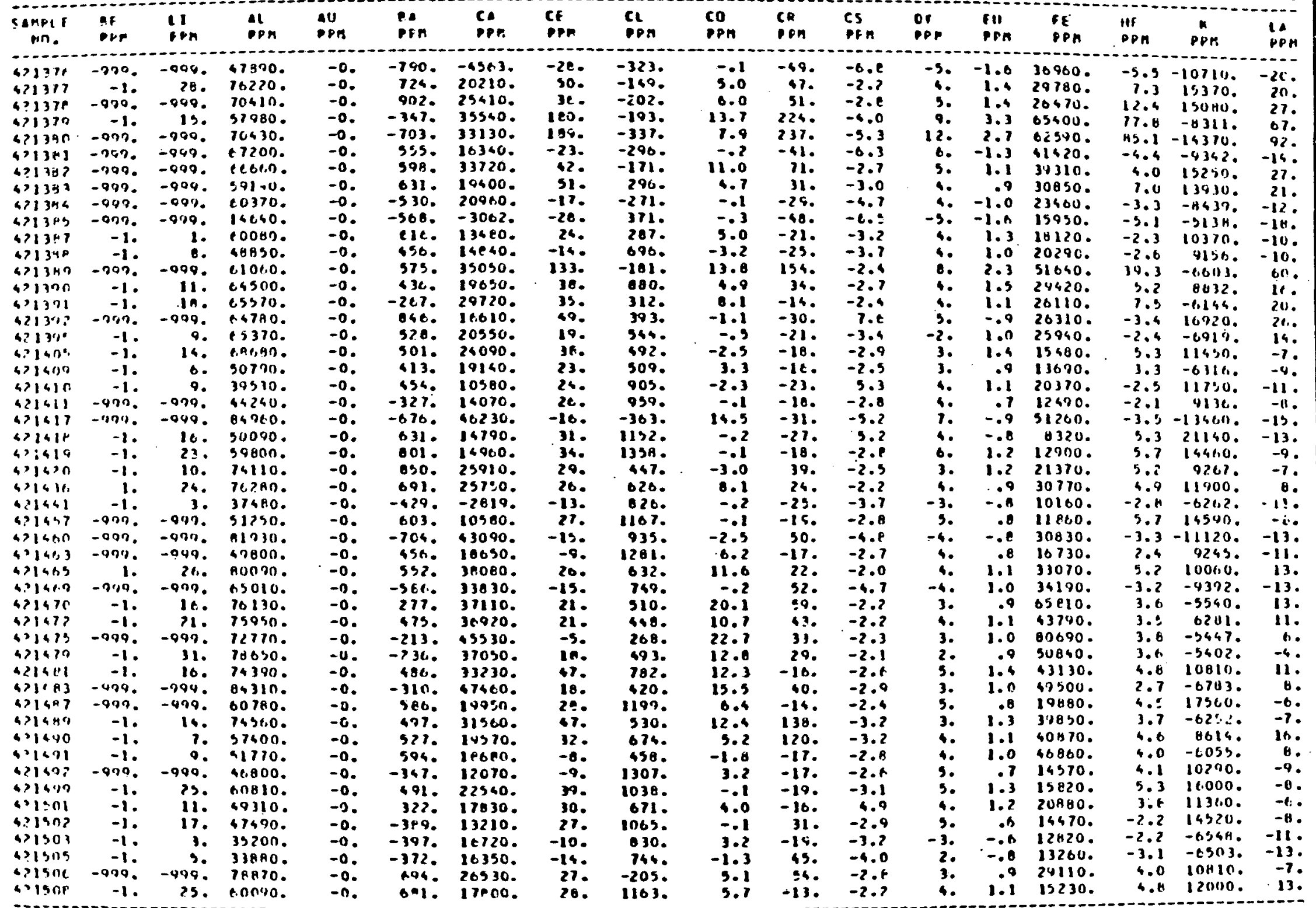




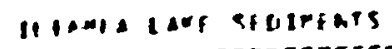

Br. InLEl - Saitile 3

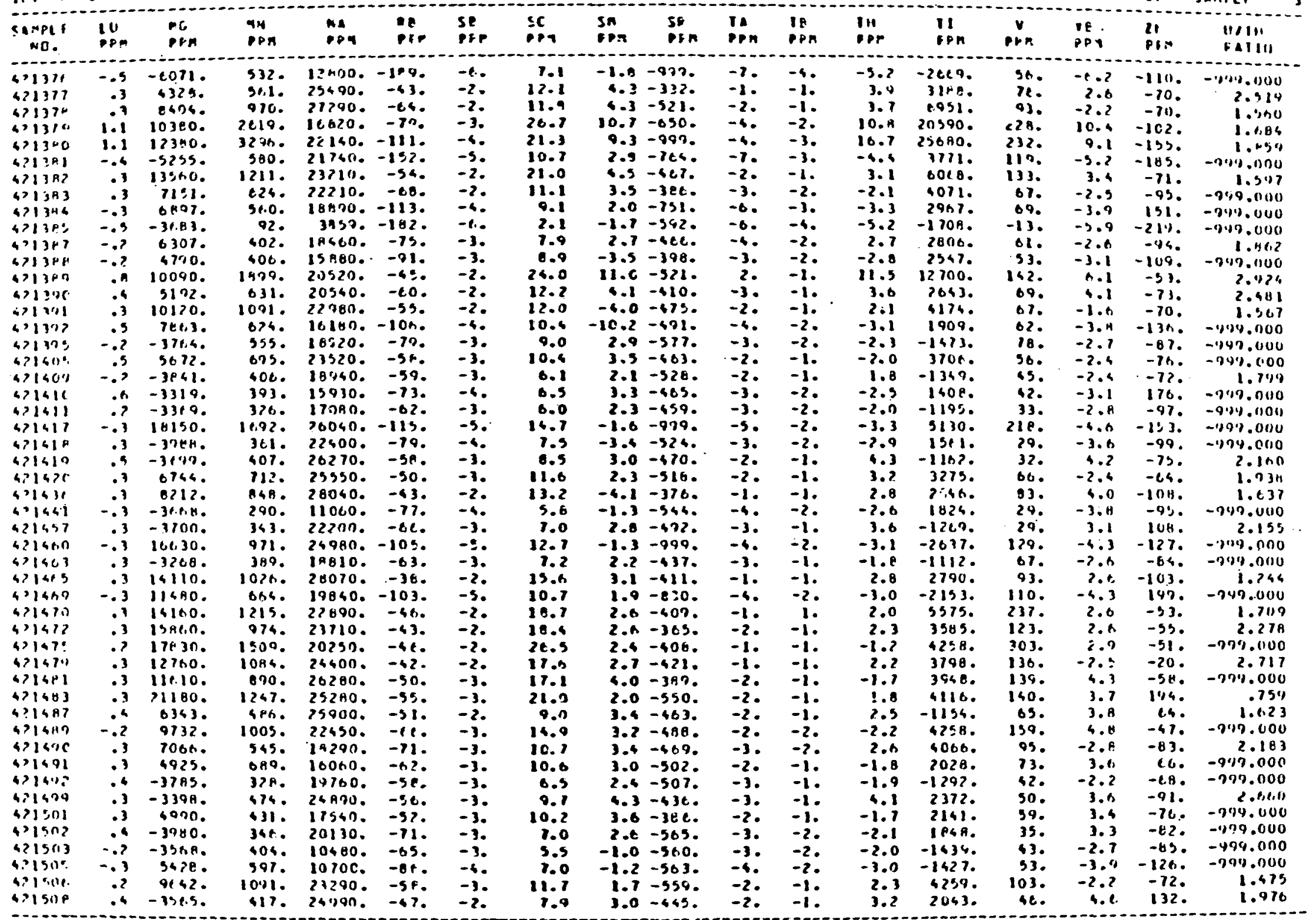


Hentia lane sroinfmis

C6. line - samice sampic la lo lone.

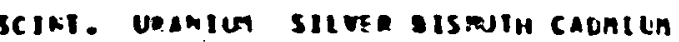
pip opr on canting copoen wion jug hiun niciel 110 lin limasien mr

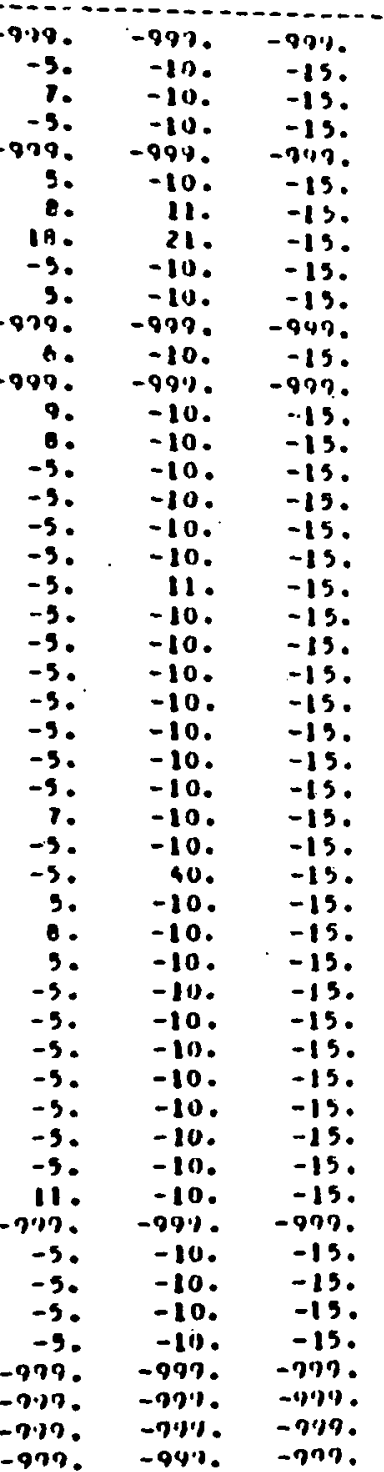

$5150950.5372 \quad 1.36 .7464$ ? 151059.9068 136.1932 421311 59.A1 154.1169 परा512 50.1102 154.2250 प2 3513 59.1.33 136.3048

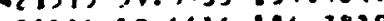
पराsin 90.1434154 .3839 Clisir 59.6214154 .4356

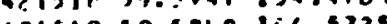

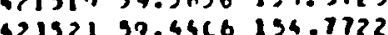
4152139.4426 194.7722 पर 157450.3746 154. 5604 ज7

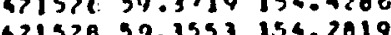
काIS2A 97. 35331.54 .2019

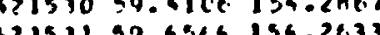
41531 97.4564156 .2633 4) 153? $59.46,691: 4.1861$ 42151550.6561153 .9125 4215119.9 .5233153 .9217 $42153739.3 \% 97183.5228$ $4156399.600 \mathrm{~h} 133.4361$

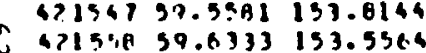
$415 \%, 59.6778$ 13.3.314 $4135450.71{ }^{201} 193.2520$ 42153597.7417193 .1797 42156099.7006153 .1394 \%158.A 57.1225 153.0339 47157559.0439153 .5146 4131959.4002133 .9501 (21391 59.3A3n 194.0192 42159759.3631156 .009 ? $4215 A 339.3017$ 196.12P3 4 113RP 59.3344194 .2902 42159059.1100134 .4206 6159350.3713154 .6707 4153439.1475154 .7122 ?1597 39.4041 156.en14 4150050.610 A 156.0478 4 4.1579 57.6033 135.0136 $416 n$ त 39.4733155 .1067 67160159.4111155 .1033 $4.160259 .4050 \quad 193.20 A 6$ arinns 50.3267155 .3606 4160759.3147195 .3264

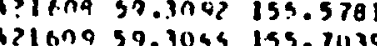

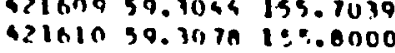

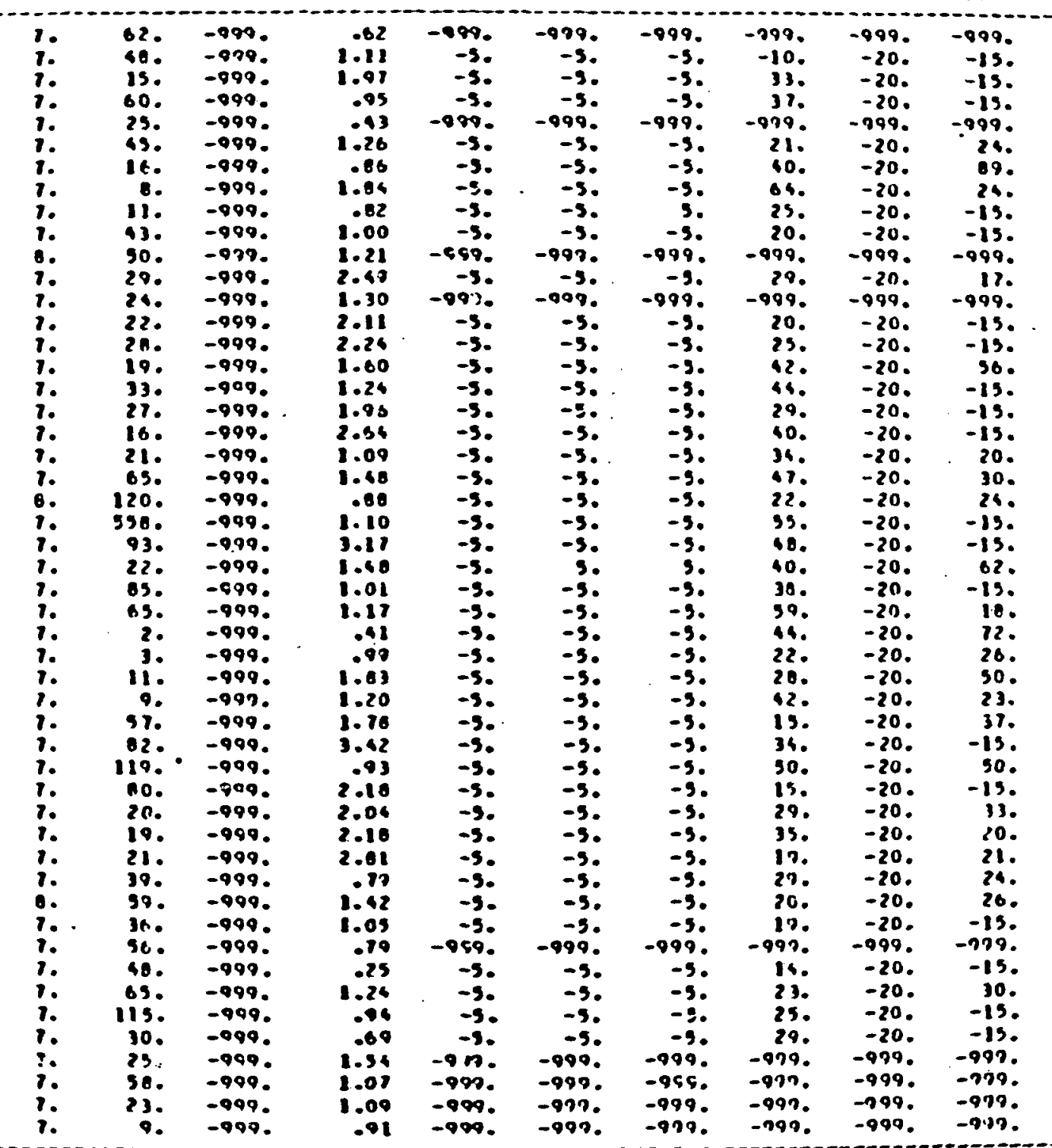

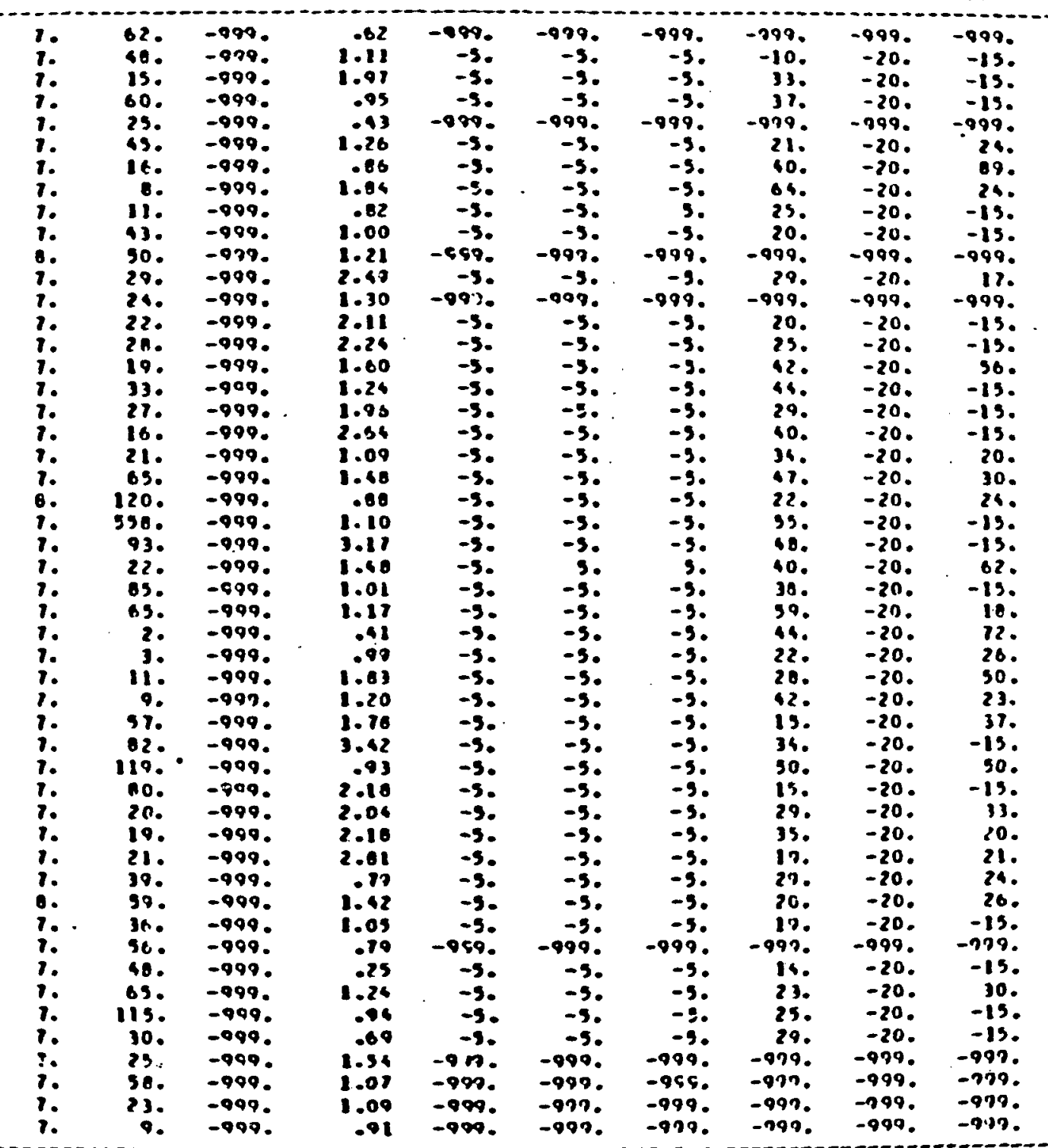

$-94.5$. 
hiane ear stojernis

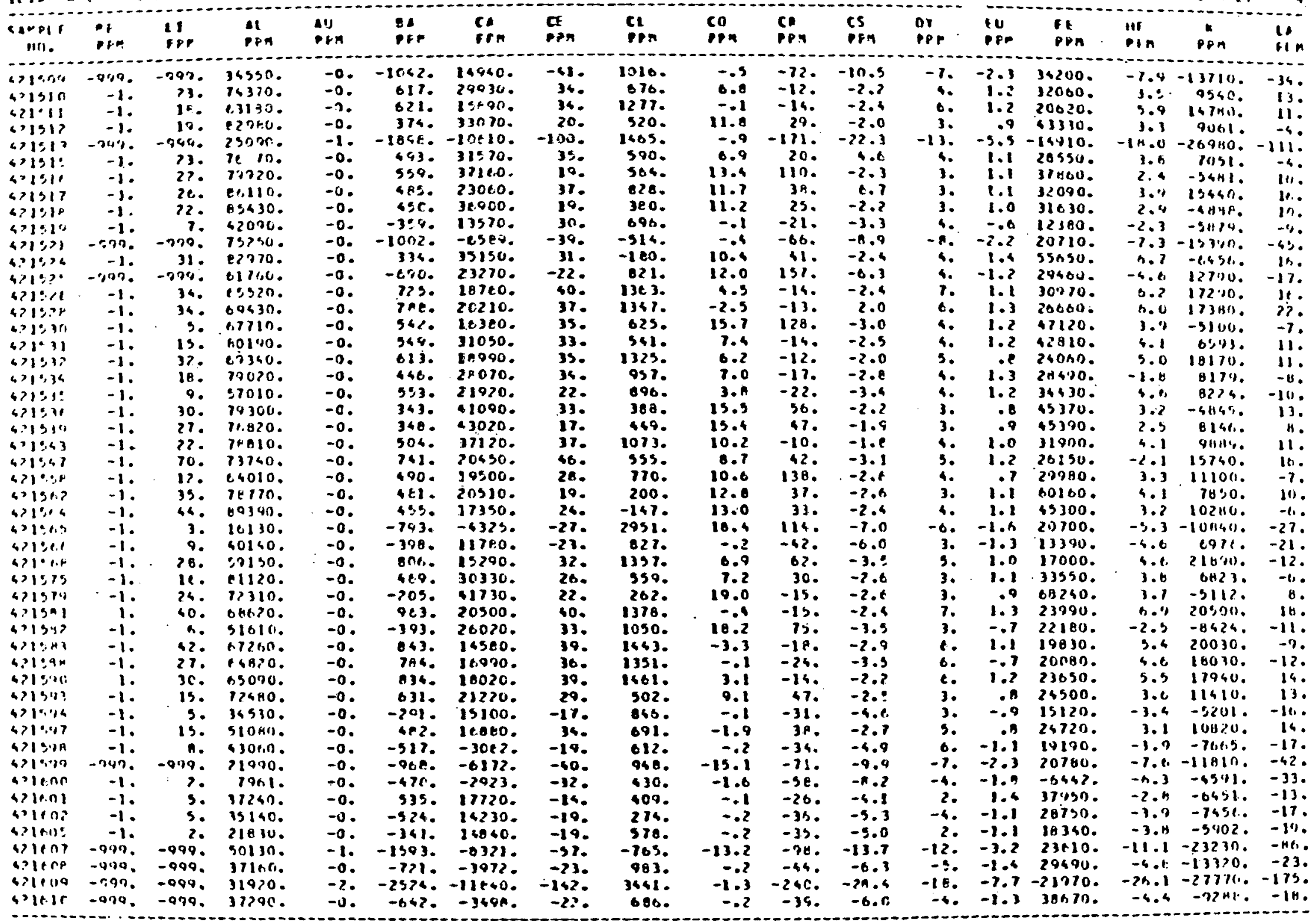


le isare eave sonierers

C. Intrl - samber

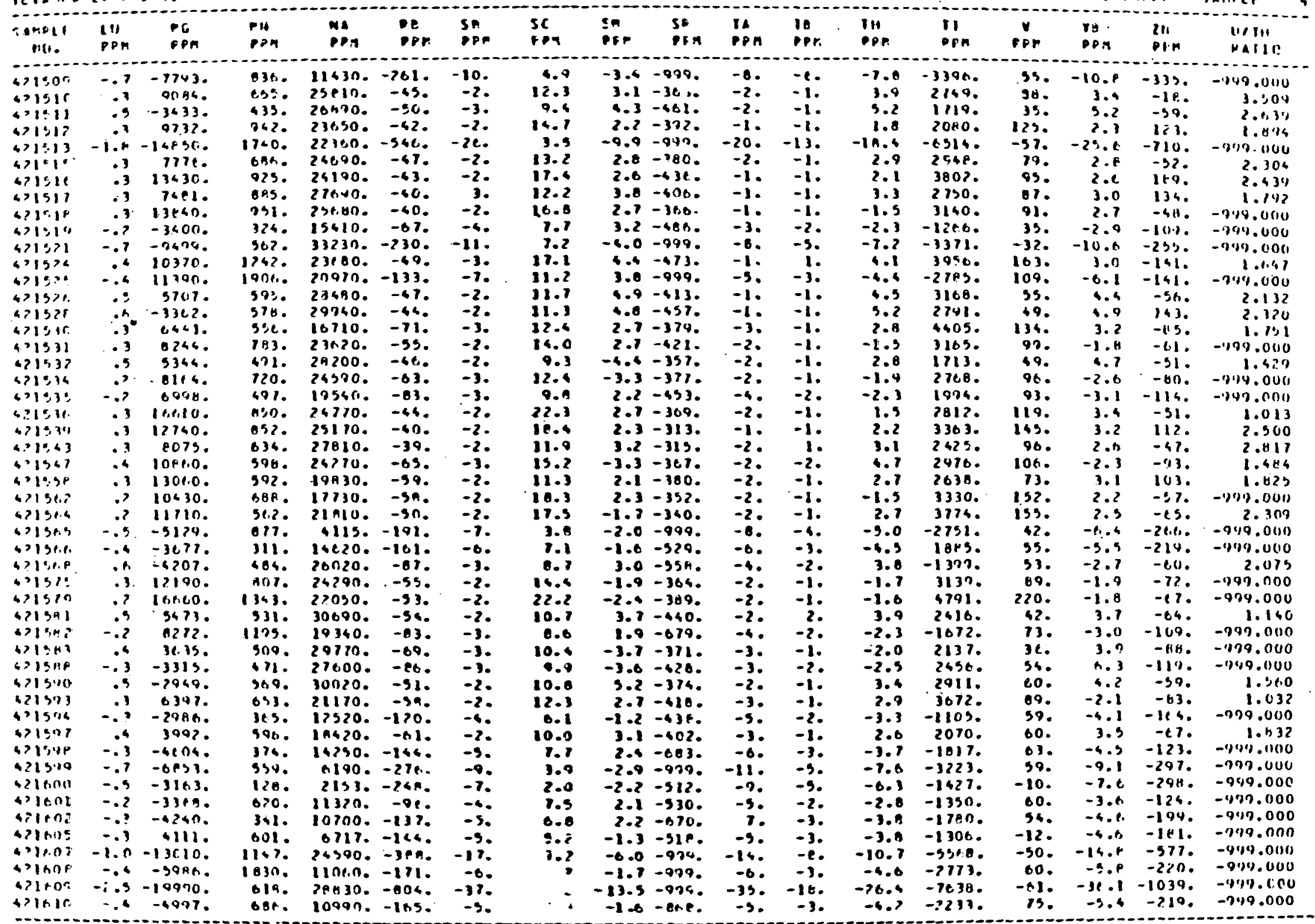




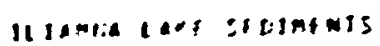

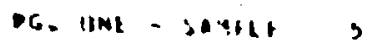

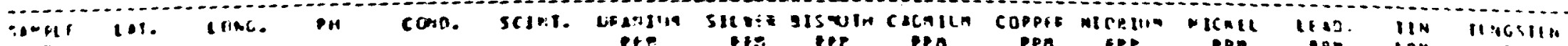

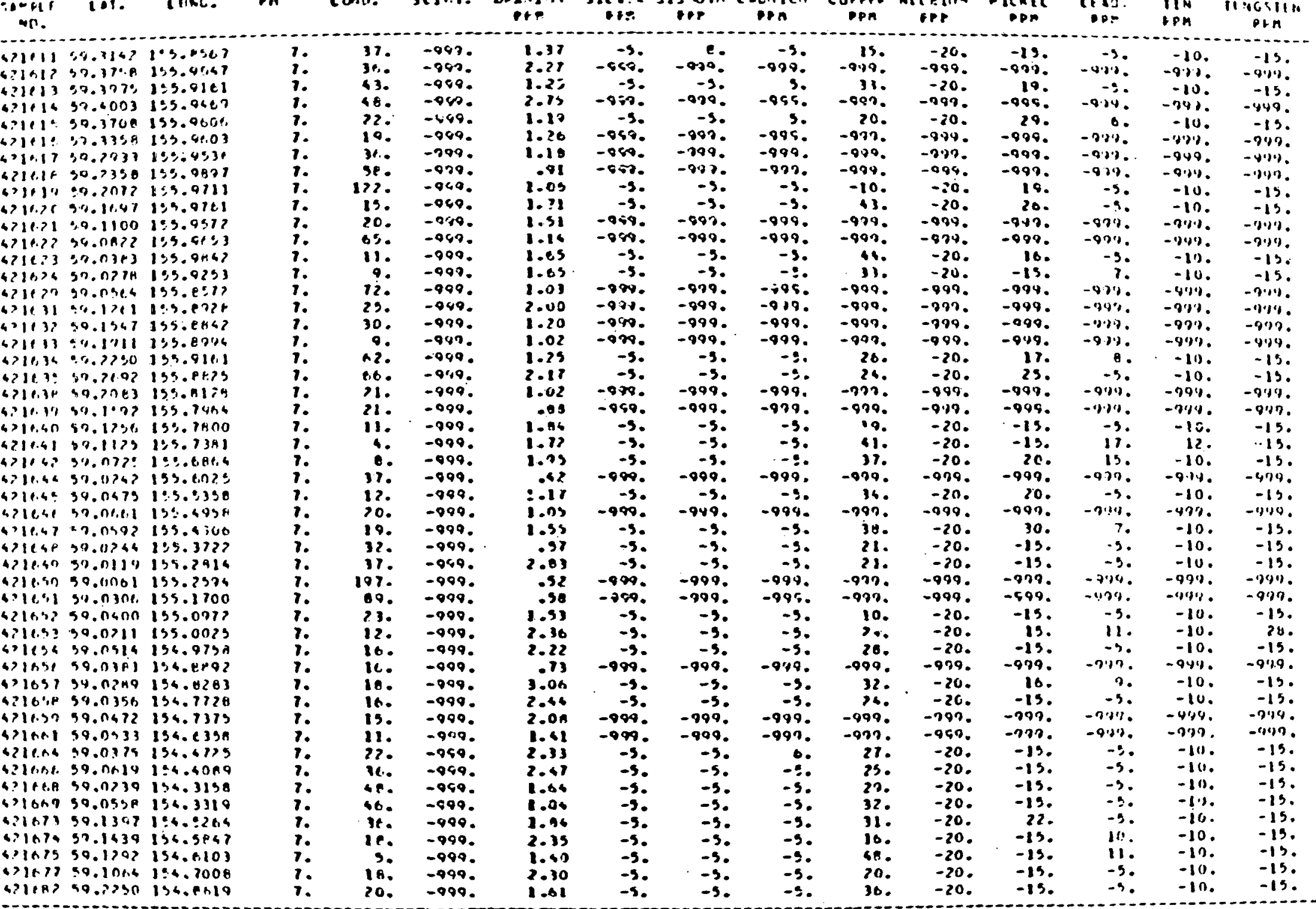




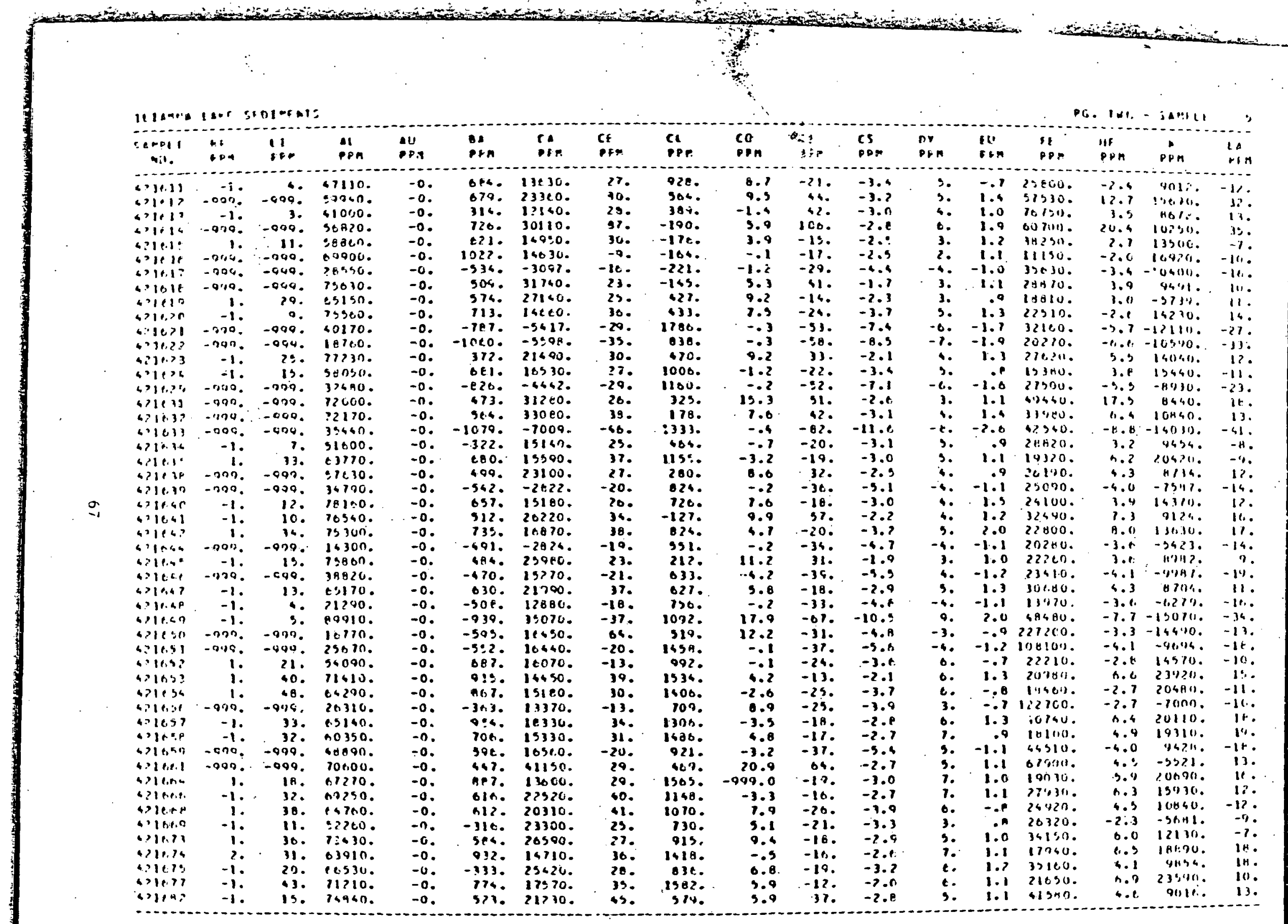




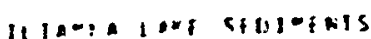

Ci. 1.1411-iand

\begin{tabular}{|c|c|c|c|c|c|c|c|c|c|c|c|c|c|c|c|c|c|}
\hline $\begin{array}{c}\cdots=1 \\
m=1\end{array}$ & I11 & is & n.t & He & it & is & SC & $\leq p$ & so & $\lim _{0 \times n}$ & $\begin{array}{l}\text { IP } \\
p>n\end{array}$ & $l_{P P P}$ & $\begin{array}{l}11 \\
\text { tor }\end{array}$ & VPp & iE & Pin & $\begin{array}{l}\text { "II" } \\
\forall 1111\end{array}$ \\
\hline 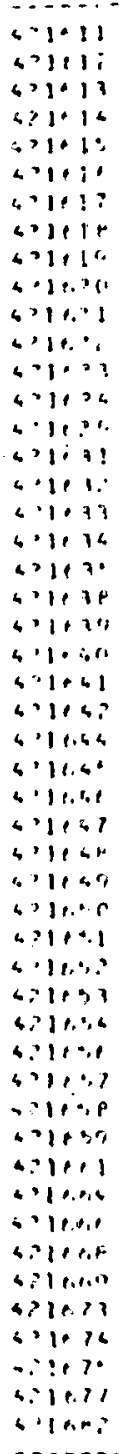 & 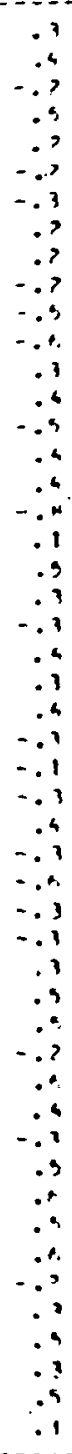 & 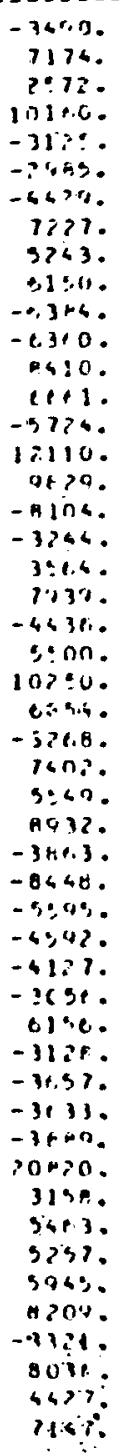 & 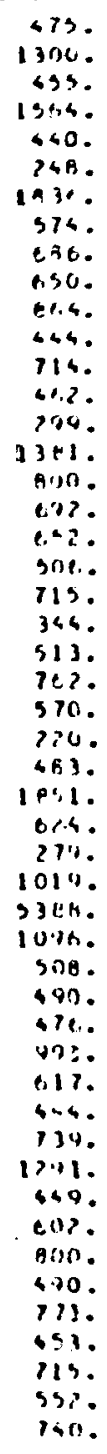 & 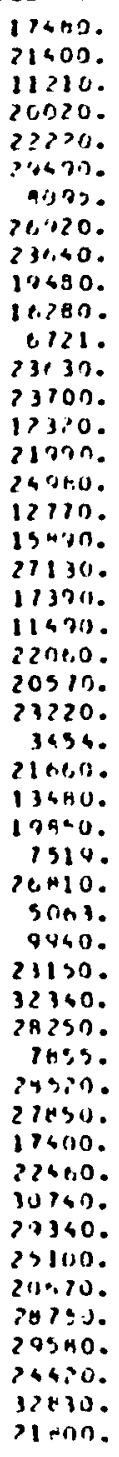 & 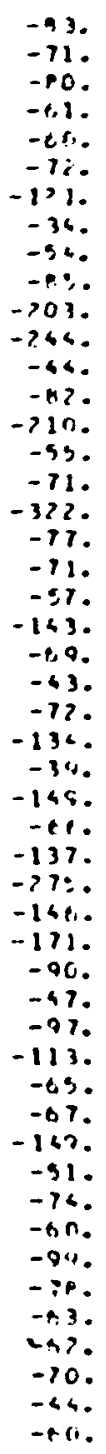 & 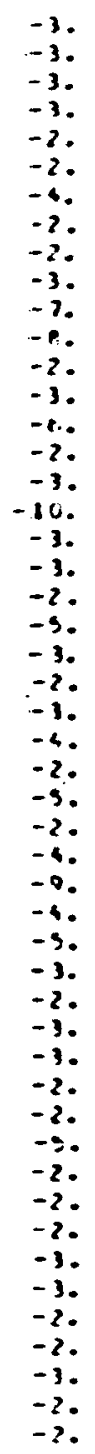 & 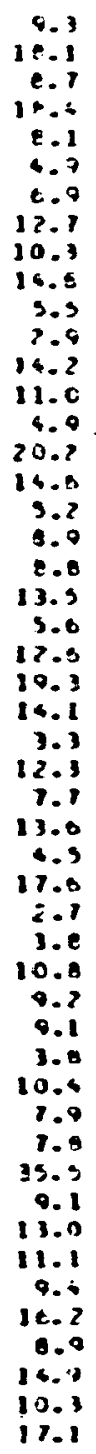 & 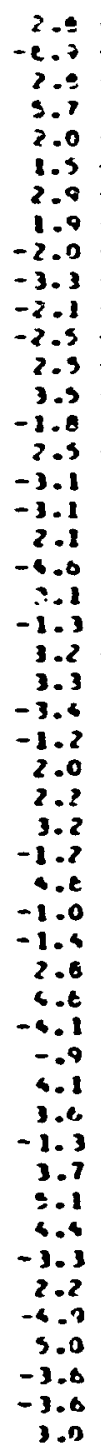 & 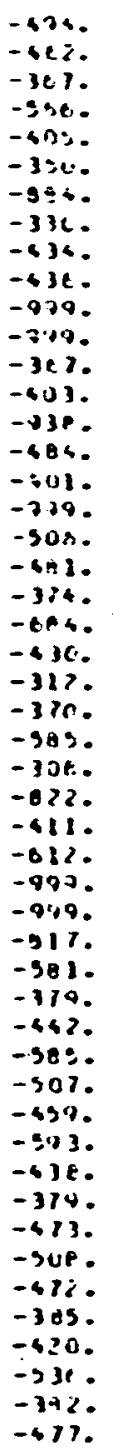 & 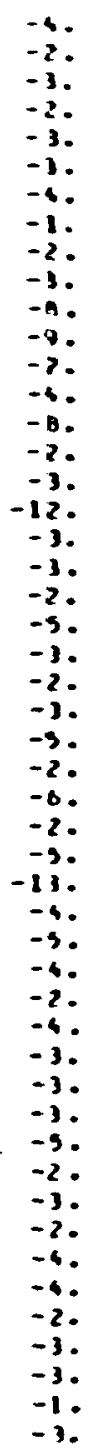 & 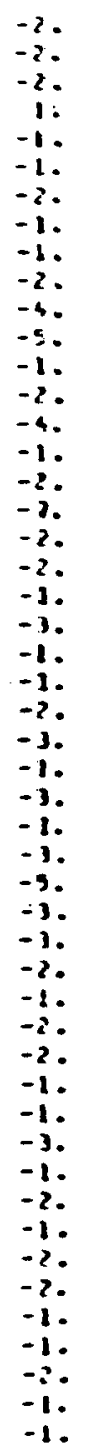 & 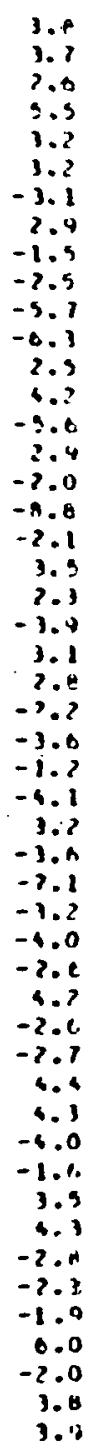 & 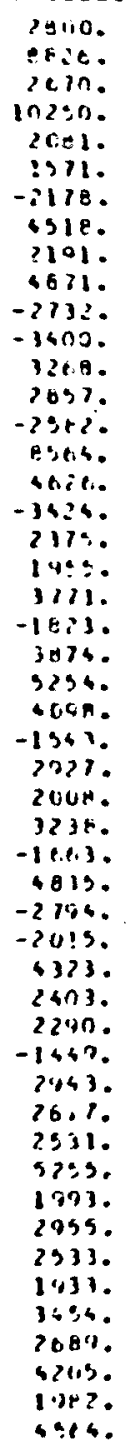 & 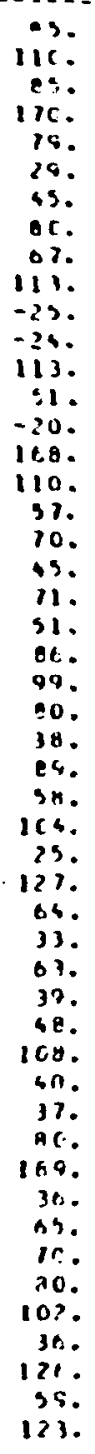 & 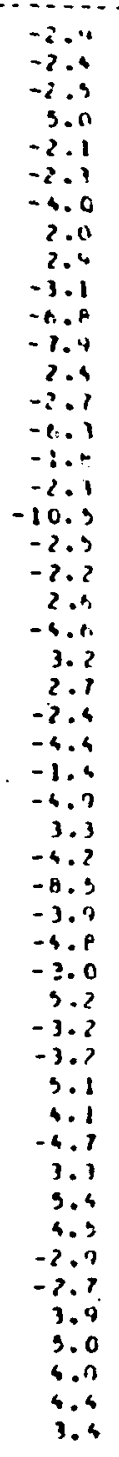 & 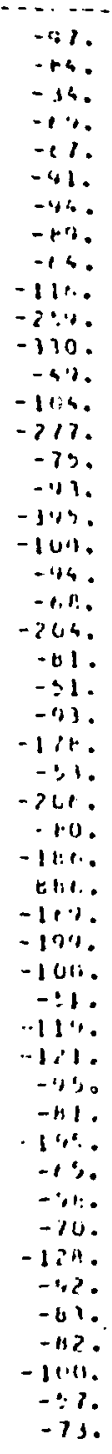 & 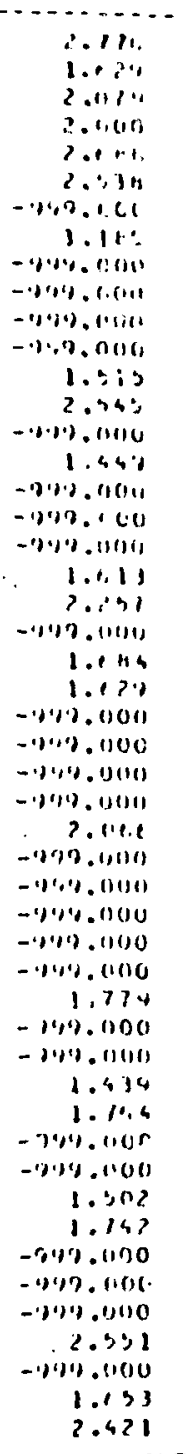 \\
\hline
\end{tabular}


110.1. 1.08 in nienas

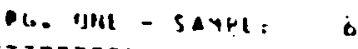

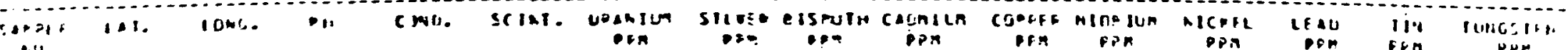

$\rightarrow 20$

or

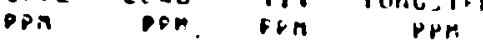

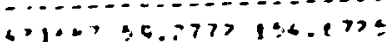
19.48 $90.7+7: 10.7946$ idede so.3ris3 10.4.304 $410 \% 97.066 ? \quad 194.3136$

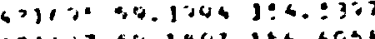

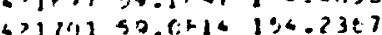
$4197 \times 0.7240$ 134.67!1 $110-40 . \operatorname{sint} 133 .+108$

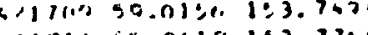

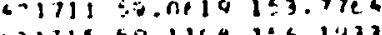

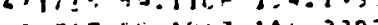
ग1717 50.1707 1:-.2703

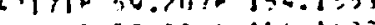
'ग110 5 2.7266 136.

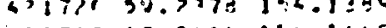

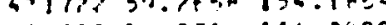

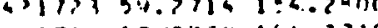
4 17.r. so.7ror li,k.3719 $\therefore 1117.53 .71033136 .60106$ 6) .

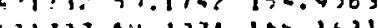
(1, 4.

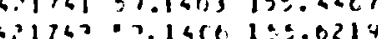
617620.14601906214 41746 90.160n 194.e? ?a

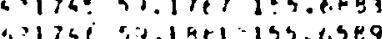

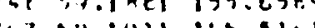

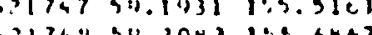

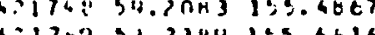
4) 39. $411 \% 90.200 \mathrm{n}$ 199.2003 4179450.7614195 .1711

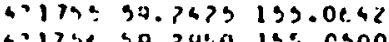
-174 40.240019500300 ?1797 94.7443 1:9.1033

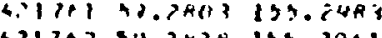

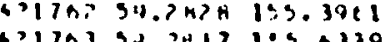

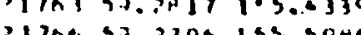
?ine $39.730 \mathrm{~N} 139.39 \mathrm{er}$

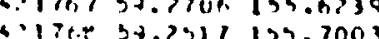
$4118,290$. itun 125.711?

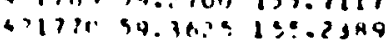

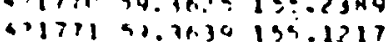

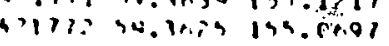

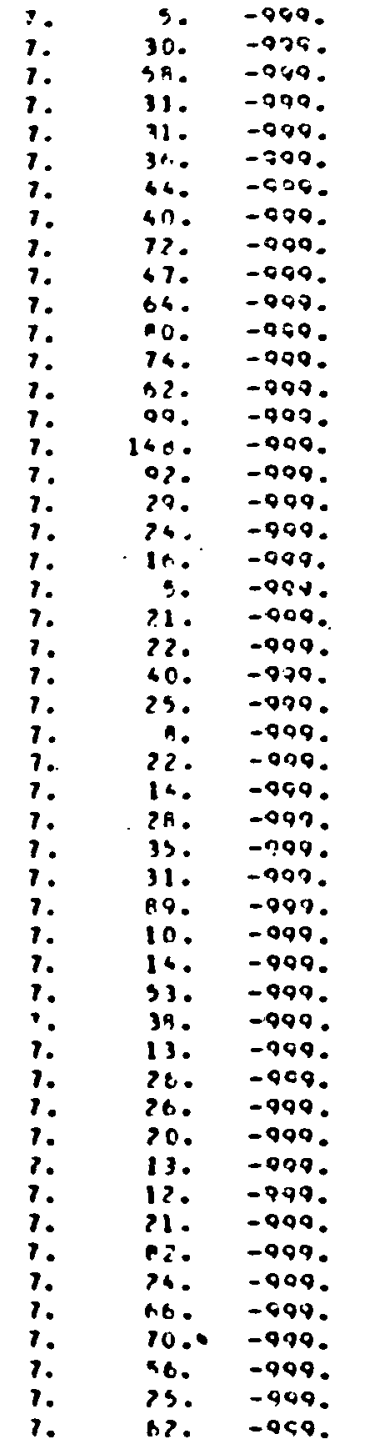

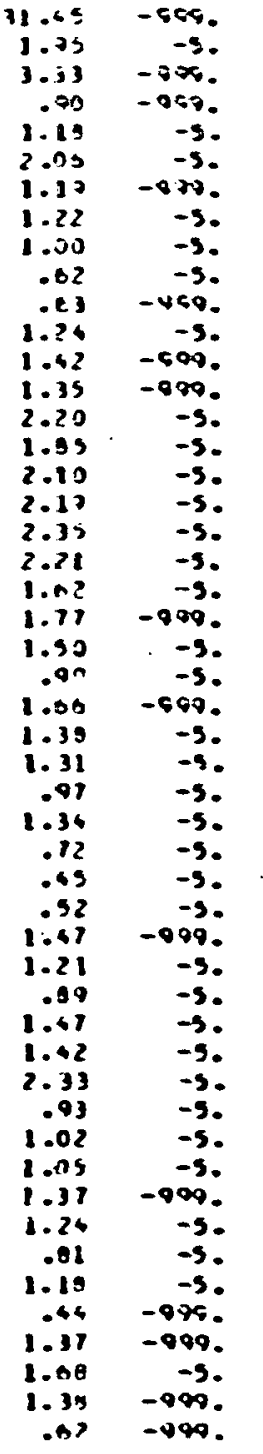

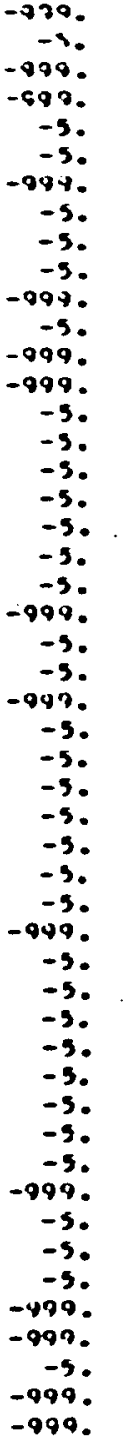

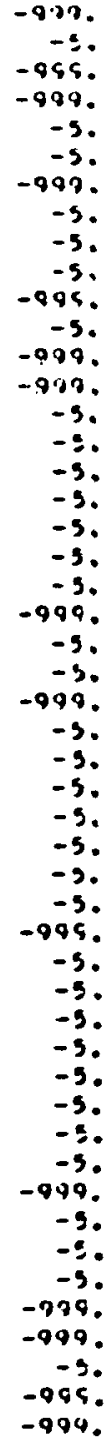

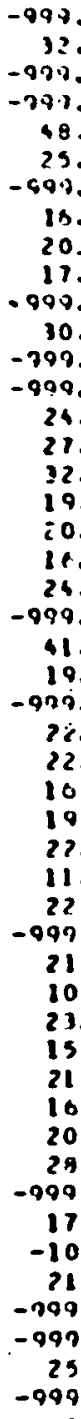

$-989$.

$-848$. $7.89 . \quad \because 149.090$ 


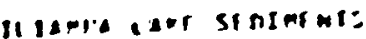

Pr. INL - sAYFLI

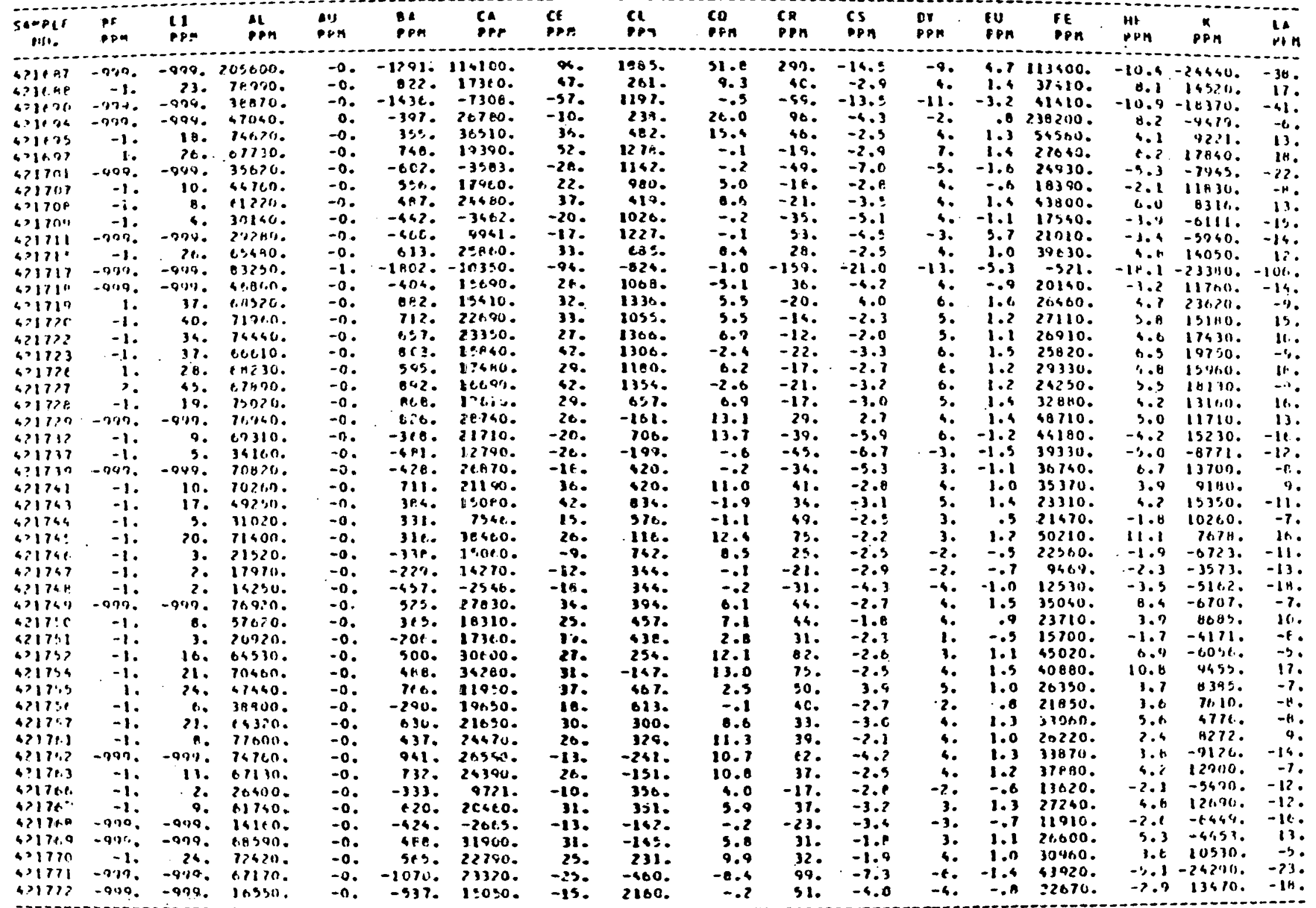




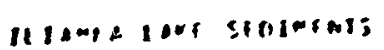

-e. liner - saible o

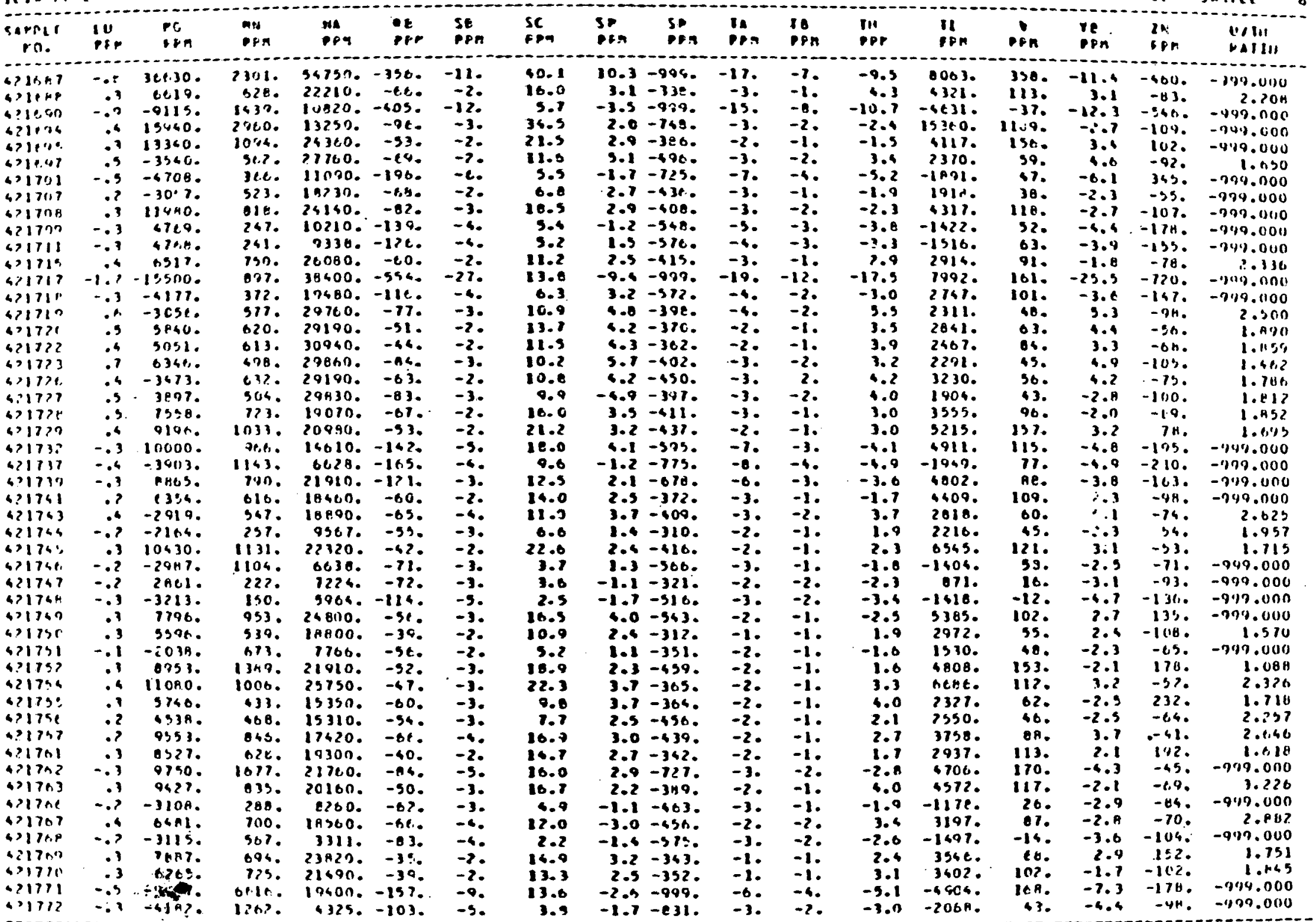




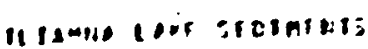

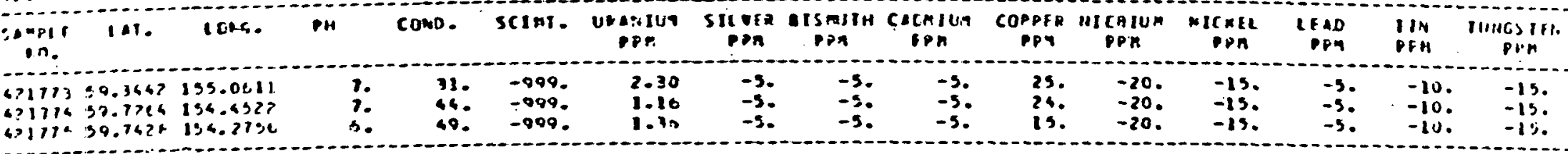

nerama laer sholments

PC. INE - SAMPLA

\begin{tabular}{|c|c|c|c|c|c|c|c|c|c|c|c|c|c|c|c|c|c|}
\hline $\begin{array}{c}\text { enprer } \\
\text { Mrs. }\end{array}$ & $\begin{array}{l}\text { HF } \\
P D M\end{array}$ & $\begin{array}{l}18 \\
P P M\end{array}$ & At & $\begin{array}{l}\text { AU } \\
\text { PPS }\end{array}$ & PR & CA & ce & $\operatorname{Cl}_{\text {PPn }}$ & $\begin{array}{l}\text { CO } \\
P P A\end{array}$ & $\begin{array}{l}\text { CR } \\
\text { PPA }\end{array}$ & $\begin{array}{l}\text { Cs } \\
\text { pon }\end{array}$ & $\begin{array}{l}\text { or } \\
\text { opr }\end{array}$ & $\begin{array}{l}\text { EU } \\
\text { PPY }\end{array}$ & $\begin{array}{l}\text { FE } \\
\text { PPA }\end{array}$ & $\operatorname{lIF}_{P P H}$ & $\stackrel{R}{P P H}$ & If. \\
\hline $\begin{array}{l}671713 . \\
421774 \\
4>1779\end{array}$ & $\begin{array}{l}-1 . \\
-1 . \\
-1 .\end{array}$ & $\begin{array}{l}8 . \\
\text { i月. }\end{array}$ & $\begin{array}{l}44340 . \\
40680 . \\
43430 .\end{array}$ & $\begin{array}{l}-0 . \\
-0 . \\
-0 .\end{array}$ & $\begin{array}{r}321 . \\
-220 . \\
582 .\end{array}$ & $\begin{array}{l}16900 . \\
15800 \\
13210 .\end{array}$ & $\begin{array}{l}12 . \\
27 . \\
28 .\end{array}$ & $\begin{array}{l}167 . \\
088 . \\
1124 .\end{array}$ & $\begin{array}{l}-.1 \\
3.7 \\
-.1\end{array}$ & $\begin{array}{l}35 \\
-14 . \\
-21 .\end{array}$ & $\begin{array}{l}-2.4 \\
-2.4 \\
-3.8\end{array}$ & $\begin{array}{l}3 . \\
3 . \\
3 .\end{array}$ & $\begin{array}{l}1.2 \\
.7 \\
1.3\end{array}$ & $\begin{array}{l}17790 . \\
14450 . \\
12170 .\end{array}$ & $\begin{array}{r}3.0 \\
-1.2 \\
4.7\end{array}$ & $\begin{array}{l}-56 ? 0 . \\
-360 \% . \\
10660 .\end{array}$ & \\
\hline
\end{tabular}

4717?

II IAYH: IAKF SEDITCMIS

PC. IllHEt - SAHILf

\begin{tabular}{|c|c|c|c|c|c|c|c|c|c|c|c|c|c|c|c|c|c|}
\hline $\begin{array}{c}\text { AHEIS } \\
\text { Nr. }\end{array}$ & 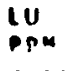 & $\begin{array}{l}\text { Pr. } \\
\text { PPM }\end{array}$ & $\begin{array}{l}\text { MN } \\
\text { PPA }\end{array}$ & $\begin{array}{l}\text { MA } \\
\text { PPM }\end{array}$ & $\begin{array}{l}R B \\
P P M\end{array}$ & SA & $\begin{array}{l}\text { SC } \\
\text { PPA }\end{array}$ & $\begin{array}{l}\text { Sn } \\
\text { PPn }\end{array}$ & $\begin{array}{l}\text { SP } \\
\text { PFH }\end{array}$ & $\begin{array}{l}\text { IA } \\
P P \text { K }\end{array}$ & $\begin{array}{l}\text { le } \\
\text { PPn }\end{array}$ & $\operatorname{lil}_{\text {POP }}$ & $\begin{array}{l}I 1 \\
P+n\end{array}$ & $\begin{array}{c}y \\
P P n\end{array}$ & $\begin{array}{l}Y E \\
P P M\end{array}$ & $\begin{array}{l}2 N \\
P B \cdot M\end{array}$ & $\begin{array}{l}\text { UCTII } \\
\text { DAIIIU }\end{array}$ \\
\hline $\begin{array}{l}6: 17173 \\
6 ? 1774 \\
4 \geq 1778\end{array}$ & $\begin{array}{r}.3 \\
-.1 \\
-.2\end{array}$ & $\begin{array}{r}6174 . \\
-3152 . \\
-3299 .\end{array}$ & $\begin{array}{l}358 . \\
409 . \\
352 .\end{array}$ & $\begin{array}{l}15.780 . \\
14800 . \\
19910 .\end{array}$ & $\begin{array}{l}-59 . \\
-51 . \\
-65 .\end{array}$ & $\begin{array}{l}-3 . \\
-3 . \\
-6 .\end{array}$ & $\begin{array}{r}10.0 \\
6.6 \\
6.6\end{array}$ & $\begin{array}{l}2.4 \\
2.3 \\
4.5\end{array}$ & $\begin{array}{l}-444 . \\
-437 . \\
-437 .\end{array}$ & $\begin{array}{l}-2 . \\
-2 . \\
-3 .\end{array}$ & $\begin{array}{l}-1 . \\
-1 . \\
-2\end{array}$ & $\begin{array}{r}5.3 \\
-1.6 \\
-2.3\end{array}$ & $\begin{array}{l}2333 . \\
1814 . \\
1795 .\end{array}$ & $\begin{array}{l}31 . \\
41 . \\
31 .\end{array}$ & $\begin{array}{r}-2.5 \\
2.6 \\
-3.3\end{array}$ & $\begin{array}{l}-36 . \\
-60 . \\
-78 .\end{array}$ & $\begin{array}{r}2.304 \\
-279.000 \\
-279.1100\end{array}$ \\
\hline
\end{tabular}




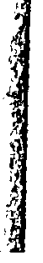

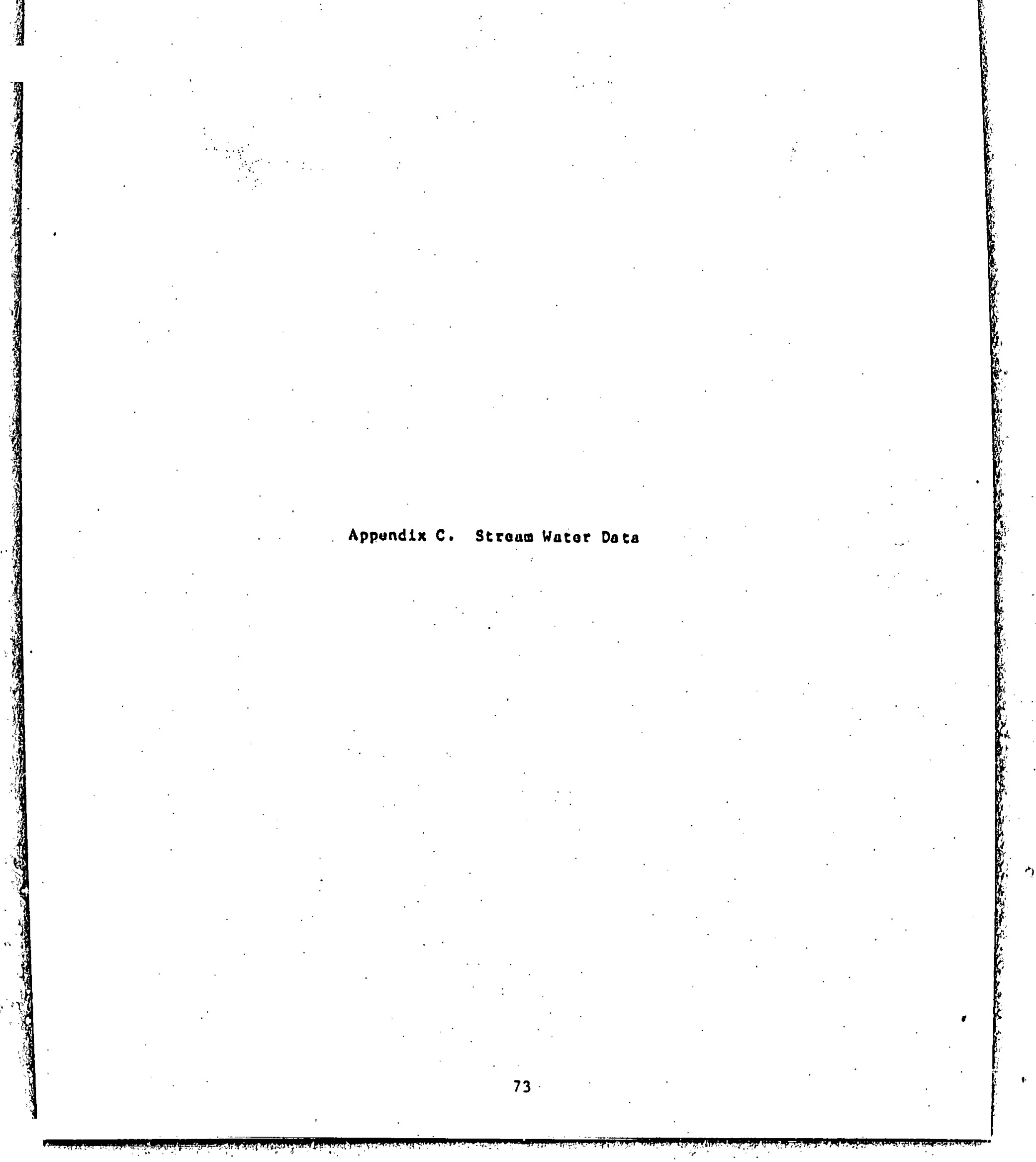


Appendix C. Streas ister Data

\begin{tabular}{|c|c|c|c|c|c|c|c|c|}
\hline $\begin{array}{l}\text { SANDLF } \\
B D E\end{array}$ & $\begin{array}{c}\text { So-vis } \\
\text { wn. }\end{array}$ & testrunt & inucsions & 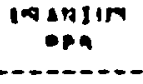 & - H & $\begin{array}{l}\text { Cnun. } \\
\text { oxmensien }\end{array}$ & $\begin{array}{l}\text { ScInt: } \\
\text { MEI cos }\end{array}$ & incoolis rusn. \\
\hline 21 & 421274 & 30.016 & 139.016 & .090 & 6.100 & 28.000 & 14.000 & 2.951 \\
\hline$? 7$ & 471271 & 50.931 & 153.051 & .030 & 7.300 & 50.000 & 1.000 & .943 \\
\hline$? 7$ & 421220 & 50.9196 & 153.010 & $-3 * 0$ & 8.400 & 0.000 & 1.000 & 4.150 \\
\hline 27 & $42122^{\circ}$ & 30.017 & 133.071 & .110 & 1.400 & 39.000 & 7.000 & 1.616 \\
\hline 27 & 421230 & 50.411 & 133.153 & .240 & 2.300 & $10 . \cos 0$ & 9.000 & 6.000 \\
\hline 21 & 421231 & 32.219 & 153.000 & .530 & $7 . \pm 00$ & 80.000 & $7.0 r_{i} 0$ & $3.9 \times 0$ \\
\hline$? 7$ & 471232 & 50.005 & 153.151 & .080 & 7.400 & $7 \% \cdot \operatorname{coc}$ & 3.0100 & .100 \\
\hline$? 7$ & $4 \geq 1733$ & 30.001 & 133.237 & .010 & 2.400 & 11.400 & 5.000 & .141 \\
\hline 3 & 421234 & 50.041 & 153.771 & .070 & 7.100 & 63.000 & $0 . n 00$ & 1,111 \\
\hline 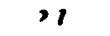 & 121234 & 50.103\% & 153.320 & .070 & 7.400 & 14.000 & 3.0130 & 1.591 \\
\hline 27 & 421234 & $52 . \times 22$ & 153.200 & $.02 n$ & 7.430 & $30 \cdot 000$ & 0.000 & $2.10 \mathrm{~A}$ \\
\hline$i$ & $421>31$ & 50.913 & 153.323 & .090 & 3.000 & 50.000 & 5.000 & 1.420 \\
\hline 7.7 & $421 ? 37$ & 09.732 & 133.331 & .010 & 7.300 & 91.000 & 7.000 & .104 \\
\hline$\geq 7$ & 421248. & 59.018 & 134.671 & .010 & 7.360 & 24.000 & 9.0 no & .417 \\
\hline 21 & 421231 & 37.030 & 135.011 & ? ?O & 7.100 & 32.000 & 9.000 & 1.130 \\
\hline ?. 7 & $42126 P$ & 37.954 & $133 . a+7$ & .040 & A.coo & 35.000 & 1.000 & 1.163 \\
\hline$\geqslant 7$ & 421324 & 39.945 & 135.0 .04 & .010 & 7.000 & $2 A .000$ & $0.00 i$ & .157 \\
\hline 21 & 421363 & 99.100 & 155.403 & .300 & 1.000 & 39.000 & 0.000 & 12.821 \\
\hline$? 7$ & 421393 & 59.039 & 154.410 & .090 & 9.300 & 30.030 & $3.0 n 0$ & 1.061 \\
\hline 27 & 421326 & 39.003 & $154.20 \mathrm{~h}$ & .240 & 7.100 & 11.000 & $7.00 n$ & 23.014 \\
\hline 27 & 421307 & 30.991 & 154.129 & .010 & $7.3 n 0^{\circ}$ & 6.000 & 7.000 & 1.250 \\
\hline 27 & $42137 P$ & $59.97 \mathrm{R}$ & $154.00 A$ & .020 & 7.200 & 11.000 & 3.000 & I.n1A \\
\hline 78 & 421104 & 39.970 & 153.075 & .010 & 7.100 & 20.000 & 0.000 & .300 \\
\hline 27 & $4 ? 1690$ & 34.049 & 154.031 & .010 & 7.300 & 5.000 & 3.000 & 2.000 \\
\hline 27 & 4,1601 & 59.73 \% & $133.0 \mathrm{~PB}$ & .270 & 0.200 & 19.000 & 1.000 & 16.211 \\
\hline 77 & 422403 & $59.9 \geqslant 8$ & 154.203 & .270 & 7.200 & 14.000 & 0.000 & $19.2 \mathrm{AB}$ \\
\hline 27 & 471406 & 39.031 & 154.164 & .040 & 9.300 & 24.000 & 0.000 & 1.017 \\
\hline 21 & 421406 & $50.69 A$ & 154.153 & Ano & 7.300 & 1.000 & $3.0 n 0$ & 110.000 \\
\hline 27 & 421407 & SO. AAS & 134.224 & .230 & 7.200 & 12.000 & $0.00 n$ & 19.181 \\
\hline$? 7$ & $42140 A$ & 59.053 & $154.24 ?$ & .230 & 10.900 & 19.000 & 1.000 & 12.778 \\
\hline 2.7 & 421412 & 30.001 & 156.140 & .200 & 7.000 & 6.000 & 3.000 & 33.333 \\
\hline$? 7$ & 421413 & 50.028 & 134.103 & .410 & $7.200^{\circ}$ & 9.000 & 1.000 & 43.530 \\
\hline 27 & 421416 & 39.1198 & 153.093 & $.5+0$ & 7.300 & 19.000 & 1.000 & 31.111 \\
\hline 27 & 421415 & SH.Anp & 134.036 & 6.030 & 7.200 & $\$ .000$ & 0.000 & 670.000 \\
\hline 27 & 421416 & 50. AnC & 154.061 & .050 & 7.200 & 9.000 & 0.000 & 0.250 \\
\hline 27 & $4 ? 14.21$ & 52.774 & 153.017 & 4.230 & 7.100 & 16.000 & $3 . n 00$ & 302.143 \\
\hline 27 & 421422 & 39.018 & 153.729 & 1.020 & 1.200 & 15.000 & 0.000 & 69.000 \\
\hline 27 & 421623 & 59.830 & 153.AA3 & .220 & 7.200 & 1.000 & 0.000 & 31.479 \\
\hline 27 & 471424 & 59.934 & 153.015 & .250 & 7.200 & 9.000 & 3.000 & 31.250 \\
\hline 27 & 421425 & 59.712 & [53,k6] & - ino & 7.300 & 4.000 & 0.000 & $\$ 0.000$ \\
\hline 27 & 421429 & 51.007 & 153.433 & .210 & .7 .200 & 5.000 & $7.00 n$ & 47.000 \\
\hline ? & 421427 & 59.727 & 153.057 & Ino & 3.300 & 2.000 & 3.nı̣o & 0.000 \\
\hline 27 & 421629 & 59.710 & 153.470 & $.0 \times 0$ & 7.200 & 12.000 & $1.00 n$ & 2.500 \\
\hline 27 & $4>14.29$. & 30.763 & 153.416 & .200 & 7.000 & 35.000 & 1.000 & 3.714 \\
\hline 27 & $6 \geqslant 1.630$ & $34.80 \mathrm{O}$. & 153.686 & .290 & 7.100 & 115.000 & 3.000 & 2.522 \\
\hline 21 & 421431 & $59.83 A$ & 153.454 & 1.950 & 1.200 & 9.000 & 11.000 & 217.170 \\
\hline 21 & 421633 & SO. AAP & 153.506 & .020 & 7.200 & 10.000 & $7.00 n$ & 2.000 \\
\hline 20 & 121634 & 39.900 & 153.671 & .010 & 7.200 & $\$ .000$ & $5.00 n$ & 2.500 \\
\hline 27 & 421439 & 39.415 & $153.4 \mathrm{AS}$ & $11 ? 0$ & 7.990 & $5 n .00 n$ & 1.000 & 2.163 \\
\hline 87 & 421617 & $50.9 \times n$ & 153.721 & .750 & 7.7 .00 & 11.000 & $3 . n 00$ & 10.231 \\
\hline
\end{tabular}


lliard slogan wese

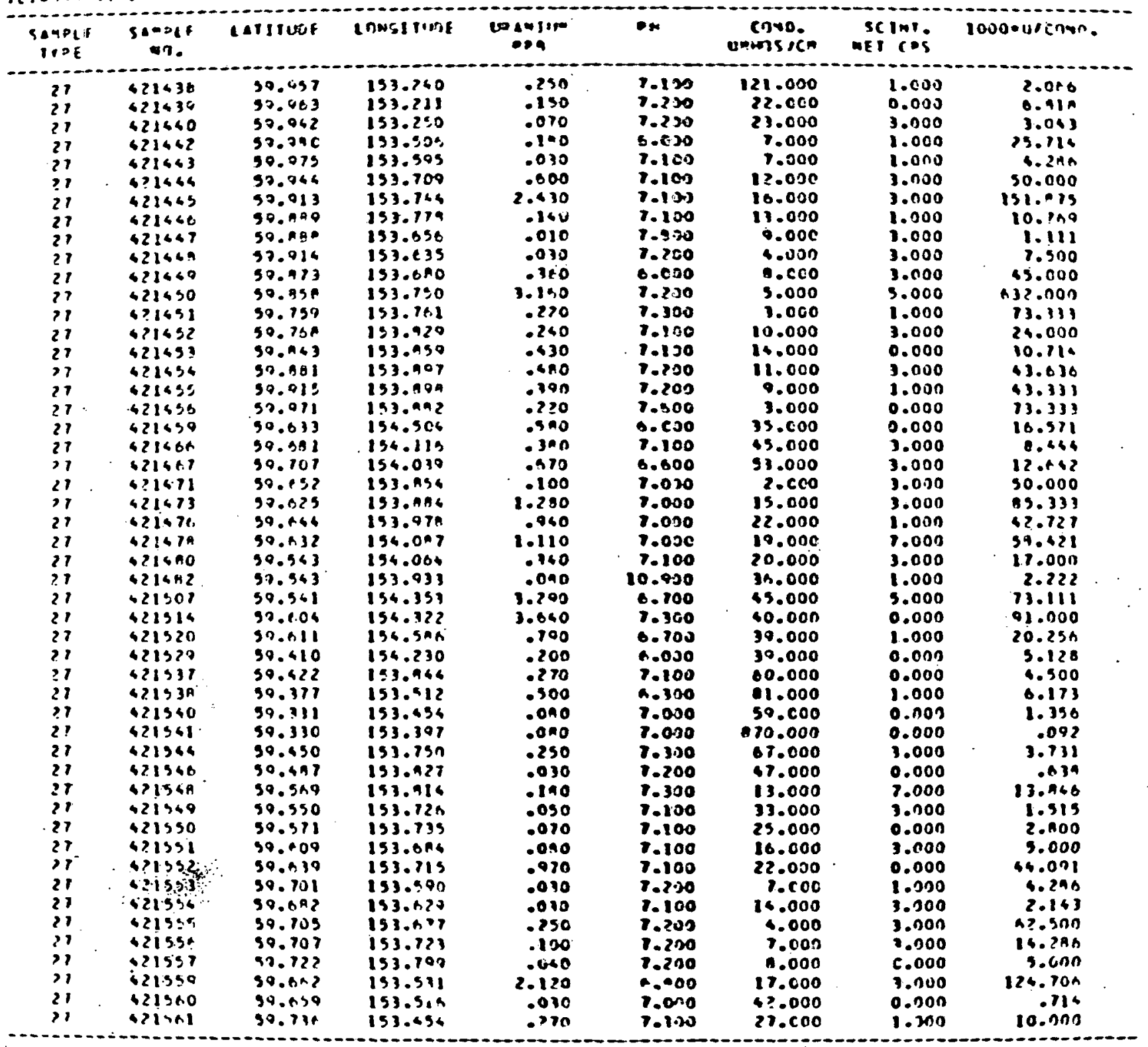




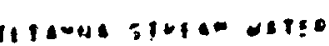

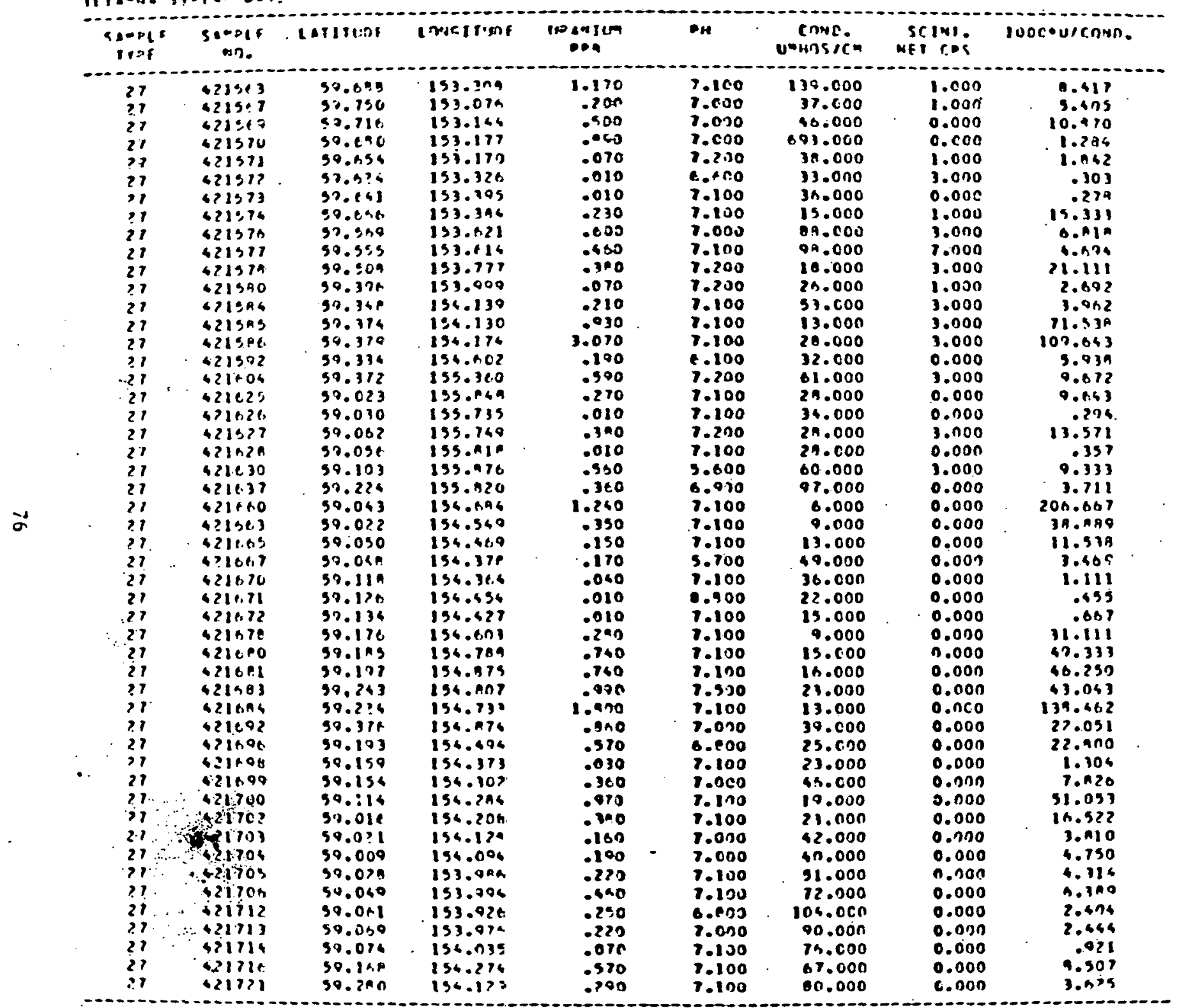




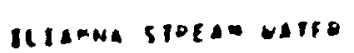

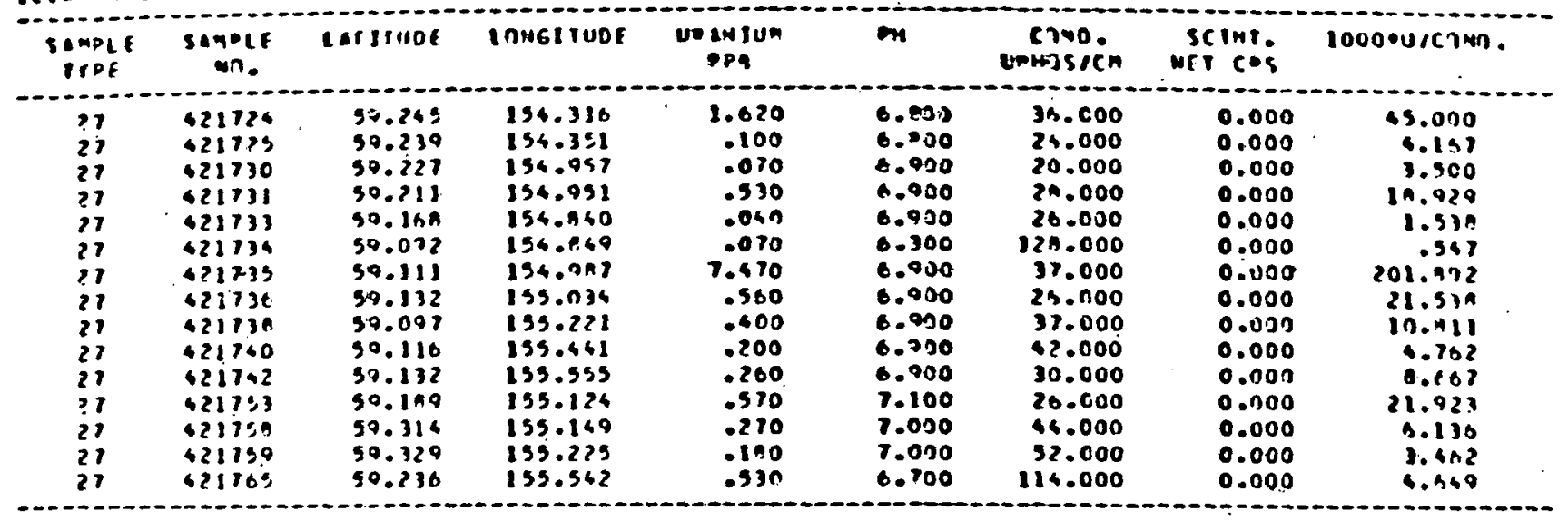




$$
-
$$


Apperalix D. Lake bater Data

\begin{tabular}{|c|c|c|c|c|c|c|c|c|}
\hline $\begin{array}{l}\text { siapis } \\
\text { irol }\end{array}$ & $\begin{array}{c}\text { Sampif } \\
\text { arre }\end{array}$ & controns & IONRIIIIDE & $\begin{array}{c}\text { igasis } \\
\text { ora }\end{array}$ & 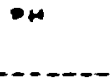 & $\begin{array}{l}\text { Cnmo. } \\
\text { exnsicn }\end{array}$ & $\begin{array}{l}\text { SCINT. } \\
\text { MET COOS }\end{array}$ & 100001110.100. \\
\hline$? 9$ & $421 \geq 39$ & 50.740 & 153.230 & .310 & 1.000 & $2 \circ .000$ & 0.000 & .191 \\
\hline 20 & $421 \geqslant 40$ & $30.7 \pm t$ & 154.709 & $.01 n$ & 7.020 & 24.000 & 0.000 & .617 \\
\hline 20 & 421261 & $50.16,9$ & 156.613 & .010 & 1.300 & 36.000 & $0.00 n$ & .776 \\
\hline 20 & 421242 & so.nos & 134.536 & .730 & $7.1=0$ & 100.0100 & 0.000 & 1.279 \\
\hline$?$ & 421263 & 50.793 & 156.463 & .010 & 7.300 & 67.000 & 0.000 & .211 \\
\hline jro & 021246 & $50.011=$ & 156.631 & +6 tho & 7.500 & II.con & 0.000 & $A I+A I A$ \\
\hline 70 & 421243 & 30.473 & 156.497 & $.23 n$ & 1.400 & 35.100 & $0.0 n n$ & $a, 571$ \\
\hline 20 & 421247 & 50.040 & 156.500 & S.ano & 7.450 & 10.050 & 0.000 & 100.676 \\
\hline 24 & $42124 A$ & 50.910 & 154.592 & .240 & 7.6500 & 33.000 & 0.030 & 1.273 \\
\hline 20 & 421260 & 59.973 & 154.633 & .010 & 7.300 & 28.000 & 0.000 & .137 \\
\hline ?ים & 421290 & 30.015 & 156.712 & .910 & 7.300 & 43.000 & 0.000 & 20.233 \\
\hline in & 471231 & 50.073 & 154.195 & .010 & 7.300 & 41.000 & 0.030 & .264 \\
\hline 22 & $421 ? 32$ & 59.024 & $154.00 \mathrm{e}$ & .010 & 1.0100 & 10.000 & 0.000 & $.95 t$ \\
\hline 20 & 421233 & 50.077 & 154.770 & - OnO & $r .200$ & $\$ 2.000$ & $0.00 n$ & $1.2 n s$ \\
\hline ?7 & 421236. & $3.9 .9 \mathrm{hA}$ & 155.012 & .010 & 1.300 & 25.000 & 0.000 & .6700 \\
\hline$? ?$ & $6 \$ 1.235$ & 50.003 & 155.1117 & .10 .0 & 7.300 & 36.000 & 0.000 & 4.664 \\
\hline $2 " 4$ & $4212: 0$ & 99.043 & 155.105 & .310 & 1.300 & 22.000 & 0.000 & 14.071 \\
\hline 11 & $4212: 7$ & $39.93 \mathrm{~A}$ & 155.164 & .310 & 1.100 & 42.000 & 0.000 & $1.3 \times i$ \\
\hline $2 \cdot 7$ & 421230 & 50.975 & 155.213 & .510 & 1.000 & 21.000 & 0.000 & 22.114 \\
\hline $2 n$ & 421250 & $39.05 A$ & 155.224 & .450 & 1.190 & 36.000 & 0.000 & 13.213 \\
\hline 20 & 421260 & $39.9 \mathrm{KP}$. & 155.320 & .590 & 7.300 & 35.000 & $0 . n 00$ & 16.937 \\
\hline 29 & 621261 & 311.049 & 153.78 .0 & .660 & 1.390 & 41.000 & $0.00 n$ & Ia.nOA \\
\hline 23 & $4212 t .2$ & 30.911 & 853.107 & .010 & $7.3 n o$ & 10.000 & 0.000 & .925 \\
\hline 29 & 421763 & $39.08,4$ & 135.656 & .170 & 7.100 & 3.000 & 0.000 & 36.ths? \\
\hline $2 \%$ & 621216 & 53.260 & 135.502 & .020 & 7.100 & 3.000 & 0.000 & 4.000 \\
\hline 20 & $(21269$ & 59.916 & 155.425 & .040 & 7.050 & 6.000 & 0.000 & 0.037 \\
\hline 20 & 421206 & 39.044 & 153.522 & .470 & 7.100 & 22.000 & 0.000 & 21.334 \\
\hline in & 421704 & 50.060 & ISS.RTB & $.0 \times 0$ & 1.300 & 27.000 & 0.000 & 2.222 \\
\hline 20 & 421270 & 39.037 & 155.164 & .010 & A.950 & 15.000 & $0.00 n$ & .AAT \\
\hline 27 & 421271 & $50.95 \mathrm{~A}$ & 155.770 & $.26 n$ & 1.000 & 1.000 & 0.000 & 31.163 \\
\hline 27 & 421272 & 30.050 & ISS.ARIO & .010 & $\$ .000$ & $3 n .000$ & $0 . n 0 n$ & .31) \\
\hline 20 & 41273 & 30.043 & $155 . n+6$ & $.1 \cap 0$ & 1.100 & 7.000 & $C .002$ & 25.116 \\
\hline 20 & 421214 & 30.933 & 155.226 & .010 & 0.900 & 42.000 & 0.000 & .73 月 \\
\hline 20 & 421273 & 30.904 & 135.016 & .050 & 1.000 & $1 \mathrm{~A} .000$ & $0.00 r$ & 3.175 \\
\hline 20 & 421270 & 90. AD4 & 135.1045 & .010 & 1.000 & 25.000 & 0.000 & .400 \\
\hline 24 & 421277 & 39.091 & 153.767 & .210 & 7.050 & 27.000 & 0.000 & $7.17 n$ \\
\hline$?$ & 421278 & $59 . A A 4$ & 155.716 &. $\sin 0$ & 7.000 & 37.000 & 0.000 & 15.135 \\
\hline 20 & 421210 & 50.919 & 155.576 & .290 & 7.100 & 19.000 & 0.000 & 15.263 \\
\hline 29 & 421200 & 30.012 & 155.529 & .730 & 1.000 & 11.000 & 0.000 & 20.002 \\
\hline 22 & $4212 n 1$ & 59.050 & 155.506 & .010 & 7.000 & 9.000 & 0.000 & 1.111 \\
\hline 20 & +21212 & 39.190 & $135.43 ?$ & .010 & 1.0 .30 & 18.000 & 0.000 & $.0>5$ \\
\hline 20 & 421203 & 30.171 & 135.351 & .010 & 7.000 & 13.000 & 0.000 & $.7+2$ \\
\hline$?$ & $421 ?+44$ & 50.941 & 155.275 & $.32 n$ & 7.0 .30 & 49.000 & 0.000 & $6.3 ? 7$ \\
\hline 20 & $4212 \times 5$ & 50.973 & iss.ina & .270 & 8.100 & 26.000 & 0.000 & 11.290 \\
\hline 20 & $4212 \mathrm{AB}$ & SQ. ARP & 155.11 ? & .000 & 7.000 & 34.000 & 0.000 & 2.847 \\
\hline 70 & $4 ? 12 A 7$ & $57.91 t$ & 155.010 & .010 & 7.100 & 43.000 & 0.000 & .?33 \\
\hline 24 & I21?AR & $59.92{ }^{\circ}$ & 154.925 & .010 & T. ino & $M .000$ & 0.000 & .130 \\
\hline 29 & $\triangle 112$ AO & 59.034 & 154.793 & .010 & $7.10^{2}$ & 41.000 & 0.000 &.$? 46$ \\
\hline 24 & 421290 & 59.930 & 154.737 & .100 & 7.3030 & 49.000 & 0.000 & 3.A78 \\
\hline 20 & $6 ? 1 ? 91$ & 30.910 & 154.AAh & 1.420 & 7.100 & 23.000 & 0.000 & 96.565 \\
\hline
\end{tabular}




\begin{tabular}{|c|c|c|c|c|c|c|c|c|}
\hline $\begin{array}{l}\text { SAMPLE } \\
\text { IIOR }\end{array}$ & $\begin{array}{c}4=218 \\
\text { wn. }\end{array}$ & catentist & Inesaslleup & 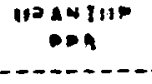 & $m$ & $\begin{array}{l}\text { Crind. } \\
\text { unnisich }\end{array}$ & $\begin{array}{l}\text { SCINT } \\
\text { NET ros }\end{array}$ & $1000011 /$ C nNA. \\
\hline $2 \%$ & 421202 & 57.017 & 154.594 & .012 & 7.109 & 23.000 & $0 . n 00$ & 2.090 \\
\hline $2 \%$ & 671223 & 90.274 & 156.011 & 3.000 & 7.100 & $E ? .0 .10$ & 0.000 & 0.065 \\
\hline$?$ & 4212064 & 57.9 .98 & 154.537 & 1.100 & 8.120 & 1.000 & $0.0 .3 n$ & 1100.000 \\
\hline$? 2$ & 421201 & $50.00,3$ & 154.951 & .012 & 7.230 & $4+.000$ & 0.000 & .717 \\
\hline $2 \%$ & $021>06$ & 52.792 & 154.709 & .790 & 7.1 .30 & $16 t .000$ & 0.0130 & 3.611 \\
\hline 200 & 411207 & 30.723 & 156.910 & .070 & 7.400 & $i 2 \cdot \operatorname{cco}$ & n.non & 1.007 \\
\hline $2 \because 1$ & $6=1 ? 04 x$ & 30.930 & 156.759 & .110 & $7.500 n$ & 52.030 & $n . n 00$ & 2.115 \\
\hline 20 & $6>1>00$ & 30.925 & 154.011 & .330 & 1.050 & 250.000 & 0,000 & .170 \\
\hline $2^{n}$ & 421300 & 57.190 & 154.603 & .360 & 7.700 & 26.000 & 0.000 & 15.19 .8 \\
\hline $7-1$ & 621301 & $57.9 n 4$ & 136.393 & .010 & 1.720 & $\$ 3.000$ & 0.020 & .233 \\
\hline ? & $421,0 ?$ & 59.063 & 154.4 .10 & $1.2 a n$ & 7.4 .20 & 10,000 & 0.000 & $12.30 \mathrm{~A}$ \\
\hline 27 & 4.1303 & 59.111 & $124 .+2+$ & .010 & 7.500 & 12.000 & 0.000 & .530 \\
\hline$? 0$ & $6 ? 1306$ & 50.017 & 196.900 & .200 & 7.230 & 17.000 & $0 . n 00$ & $11.7+5$ \\
\hline 2.1 & $6,130 \%$ & .57 .835 & 154.871 & .010 & $7 . ? 20$ & B. $0<0$ & $0 . n n !)$ & I.nal \\
\hline$? 2$ & G2I IUe & $5 ? \cdot 10$ & 154.000 & $.2 \cdot 0$ & T.100 & $29.00 i^{\circ}$ & 0.200 & 0.055 \\
\hline $7 \%$ & 471301 & 30.127 & 154.963 & $1.3 \times 0$ & 7.200 & $25.00 n$ & $0.0 n n$ & 60.629 \\
\hline$? " 1$ & 4213 CIA & 4.92 .927 & 196.056 & .190 & 7.300 & 13.000 & c.coso & 32.643 \\
\hline$p$ & 421310 & 50.170 & IS5:CA? & .520 & 7.200 & 32.000 & 0.000 & 16.250 \\
\hline 3.1 & 4111 & 37.915 & 138.030 & 1.010 & 7.100 & 45.100 & 0.010 & 22.646 \\
\hline 27 & 421312 & 99.030 & 159.111 & .940 & 7.100 & 4.000 & 0.000 & 239.000 \\
\hline 20 & 421313 & 20.832 & 155.142 & .10 .0 & 1.200 & 23.000 & 0.000 & 0.951 \\
\hline $3 " 4$ & 421316 & 39.115 & 155.173 & .700 & 7.200 & $35.00 n$ & 0.000 & $9.29 n$ \\
\hline 311 & $6 ? 1315$ & 52,110 & 155.202 & .380 & 7.200 & 42.000 & $0 . n$ isn & $9.04 \mathrm{~A}$ \\
\hline 74 & $4: 1318$ & $59.92 A$ & $153.21^{\circ}$ & - hon & 7.300 & 1.000 & 0.300 & poo. uno \\
\hline 27 & 421317 & SO.A3R & $155.31 \mathrm{~h}$ & - anso & 7.300 & 2.000 & 0.000 & $330.00 \%$ \\
\hline 27 & GIBIA & 50.911 & 153.370 & .010 & 8.100 & ".con & 0.0 .30 & 2. \\
\hline$\therefore$ & 421310 & 30.826 & 193.403 & .610 & $7.10 n$ & $\therefore .000$ & 0.000 & 13.600 \\
\hline$?$ & 421320 & S9.RIS & 155.619 & .330 & 7.1 no & 14.000 & 0.000 & 17.364 \\
\hline 2.8 & 621321 & 59.909 & 155.471 & .130 & 1.000 & 20.000 & 0.000 & 12.632 \\
\hline 27 & 621322 & 50.103 & 153.967 & .250 & 7.4 .30 & 5.000 & 0.000 & 30.0011 \\
\hline 212 & $4: 1323$ & 59.930 & 159.1004 & .100 & 8.200 & 0.000 & $0 . n 00$ & 133.331 \\
\hline 27 & 471.125 & 59.120 & $153.69 ?$ & .260 & 1.200 & A.0030 & 0.000 & 40.000 \\
\hline 3 & 421126 & 39.007 & 155.737 & .010 & 1.110 & 3.000 & $0 . \ln c$ & $2.00 c$ \\
\hline P & 421327 & 50.910 & 153.771 & .010 & 1.900 & 17.000 & $0.00 n$ & .033 \\
\hline E" & $42112 \mathrm{~A}$ & 50.631 & 135.819 & .010 & 7.900 & 14.000 & 0.000 & .116 \\
\hline$?$ & 421324 & $59 \cdot 20$ & 155.176 & .230 & 7.170 & 3.000 & 0.000 & 93.333 \\
\hline 27 & $4 \because 1330$ & 30.035 & 155.904 & .790 & 1.000 & $7.00 n$ & $0.0 n n$ & $11>.857$ \\
\hline 29 & 421331 & $50 . A 11$ & 183.947 & .010 & 1.100 & 4.000 & 0.000 & 2.900 \\
\hline$>4$ & 4? & $34.14=$ & is5.0ne & .310 & 7.100 & $\because \cos$ & 0.000 & 02.500 \\
\hline 29 & 421313 & 54.700 & 159.000 & .240 & $7.0 n o$ & 4.000 & 0.003 & 60.050 \\
\hline 20 & 421334 & 59.077 & $135.93 \mathrm{~A}$ & .010 & .1 .190 & 6.000 & $n .900$ & 2.500 \\
\hline$>0$ & 421130 & 34.019 & 153.968 & .010 & 1.100 & 3.000 & 0.000 & 3.113 \\
\hline 20 & $4 ? 1139$ & 59.112 & 155.900 & .110 & 3.650 & 2.000 & 0.000 & 9.0001 \\
\hline 20 & 621337 & 90.730 & 195.172 & .010 & 7.000 & .000 & O.C.CO & $2.000 n$ \\
\hline 20 & 421338 & 50.742 & 155.907 & .010 & $7.0 \% 5$ & 7.000. & 0.000 & 1.670 \\
\hline 1.1 & 421330 & $34.7 \geq 5$ & 155.707 & .010 & 7.000 & B.onn & $0 . n 0 n$ & 3.331 \\
\hline 29 & 421160 & 59.729 & 139.715 & .010 & 6.993 & $2 \pi \cdot \operatorname{con}$ & 0.072 & .797 \\
\hline 20 & $4 ? 1361$ & 50.112 & $155 . \operatorname{san}$ & $.0>0$ & 7.100 & 2.000 & 0.000 & $10.00 n$ \\
\hline$? 4$ & $4 ? 136 ?$ & 52. TAP & 155.932 & .010 & 7.010 & $9.00 \mathrm{C}$ & 0.000 & $\therefore a \in C$ \\
\hline$? 2$ & 421143 & 59.717 & iss.ents & $.0 ; 0$ & T.1no & 5.Dnn & n.onn & ?.nonn \\
\hline
\end{tabular}




\begin{tabular}{|c|c|c|c|c|c|c|c|c|}
\hline 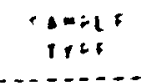 & 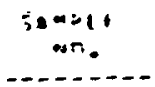 & esiliner & IJMEIIIGIF & $\begin{array}{c}\text { vespline } \\
\text { Opp }\end{array}$ & -4 & $\begin{array}{l}\text { CnNo. } \\
\text { Un+NGICD }\end{array}$ & $\begin{array}{l}\text { srinis } \\
\text { MfI cos }\end{array}$ & 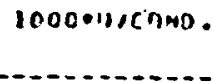 \\
\hline$?$ & 621366 & $5 \% .111$ & 153.567 & .120 & 0.25. & 3.000 & 0.000 & $? 6.000$ \\
\hline$\because$ & 421365 & $: 5.85 t$ & 1359.524 & 1.320 & $\leq .900$ & 7.5 .006 & 0.000 & 52.900 \\
\hline $2^{4}$ & 071362 & stotes & 235.435 & $.0 A O$ & 7.900 & $20 . C O C$ & 0.000 & 3.000 \\
\hline ?" & $\because \because 1367$ & $34.7=1$ & 155.306 & .100 & 7.120 & 30.000 & 0.000 & $2.11 \mathrm{~A}$ \\
\hline$?$ & 621168 & 30.111 & 155.703 & $.0+0$ & 7.100 & $22 \cdot 000$ & 0.000 & 2.121 \\
\hline ;4 & 421360 & 50.730 & 195.221 & .170 & 7.130 & $30 . \mathrm{cor}$ & 0.000 & 3.900 \\
\hline$\because$ & $4213: 0$ & $\because \because \ldots 10$ & $15: .024$ & .94 & 7.230 & 21.000 & 0.000 & 41.405 \\
\hline$\therefore$ & $4: 33: 1$ & $30.04=$ & 154.100 & 0.270 & 1.100 & 35.000 & 0.000 & 170.163 \\
\hline$\therefore$ & $4: 13: 2$ & 50.473 & 154.795 & $.1 \geq 0$ & 7.300 & 43.000 & 0.000 & 3.0 .21 \\
\hline$?$ & $4713: 3$ & 32.190 & $156.92 \%$ & .070 & 7.310 & 39.000 & 0.000 & 1.842 \\
\hline iri & $4 ? 13: ;$ & SD. $13 \mathrm{~A}$ & 156.729 & .010 & $\therefore .290$ & 3.000 & 0.000 & 3.313 \\
\hline$x$ & $4 \geq 1135$ & $=0.179$ & $136, A R_{6}$ & .010 & 7.200 & 1.000 & 0.000 & 1.429 \\
\hline$?$ & 621358 & 50.111 & 154.716 & .010 & 7.100 & 12.000 & 0.000 & .933 \\
\hline$g$ & $? 1357$ & 91.116 & 153.000 & - Ino & 1.0 .10 & 1.000 & 0.000 & 43.000 \\
\hline$?$ & $6,1,1,6$ & 97.113 & 183.167 & .11 .0 & .100 & 5.000 & 0.000 & 36.000 \\
\hline $2 x$ & 421350 & $=52.110$ & 135.364 & 1.1 .0 & 0.030 & 0.000 & 0.000 & 120.464 \\
\hline $7 "$ & $=22 i 200$ & $30 .+92$ & 195.?AD & .950 & 1.100 & 6.000 & 0.000 & $15 \pi .313$ \\
\hline$\therefore$ & $c \mid s+1$ & $50 .+.91$ & 183.326 & .030 & 1.000 & 18.000 & 0.000 & I.AB? \\
\hline$=1$ & पदा & $50.10 \mathrm{H}$ & 195.350 & .350 & 7.000 & 35.000 & 0.000 & 10.000 \\
\hline 21 & +21116 & 30.900 & 155.641 & .200 & 7.1100 & 5.000 & 0.000 & 40.000 \\
\hline$\therefore$ & $4213+3$ & 30.610 & 155.561 & $.9 \times 0$ & 1.000 & 36.000 & 0.000 & 28.735 \\
\hline$\therefore$ & 421306 & 59.615 & $153.50 \mathrm{~A}$ & .010 & $7.1 n_{0}$ & 20.000 & 0.000 & .500 \\
\hline$\because$ & $6>31.7$ & $=0 .+11$ & iss.hoe, & .210 & 7.100 & 37.000 & 0.000 & 3.676 \\
\hline $2 \%$ & $4 ? 13: 8$ & 90.0 .50 & 199.796 & $.0: 0$ & 1.200 & 2.000 & 0.000 & 3.000 \\
\hline 310 & .2110 .4 & 50.062 & 153.170 & .750 & 1.1 no & 5.030 & 0.000 & 130.000 \\
\hline 81 & 121371 & 39.041 & 135.854 & .430 & 7.000 & 21.000 & 0.000 & 21.629 \\
\hline $7 \%$ & 421371 & $90 . n>5$ & 135.070 & .460 & 1.100 & 14.000 & 0.000 & 31.429 \\
\hline 70 & 471377 & 5.8 .301 & (35.45) & 1.100 & 1.170 & 20.000 & 0.000 & 59.000 \\
\hline$\because$ & 62137.3 & 30.515 & IS5.042 & .010 & 1.190 & 10.000 & 0.000 & .536 \\
\hline 24 & 41114 & 50.350 & 195.078 & .012 & 1.100 & 9.000 & 0.000 & 1.111 \\
\hline 29 & 421375 & 39.300 & $159.0 \mathrm{~A}$ & .470 & 7.100 & 17.000 & 0.000 & 27.947 \\
\hline 14 & $62137 t$ & 50.450 & 155.0190 & .250 & 7.200 & .000 & 0.000 & .11 .250 \\
\hline 21 & 621311 & $57.3 n A$ & 155.909 & .010 & 1.100 & 8.000 & 0.000 & $1 . .30$ \\
\hline 24 & $42117 A$ & $30.3 i 7$ & 139.199 & .010 & 7.000 & 16.030 & 0.000 & -A? \\
\hline 20 & 421570 & 50.052 & $159 . \times 10$ & .170 & T.ono & 30.000 & (.oni) & 4.358 \\
\hline 10 & $\therefore: 13 \times 0$ & 50.371 & 159.701 & .010 & 7.100 & 35.000 & 0.000 & .796 \\
\hline 20 & $4 \geq \|$ If I & 54.0 .34 & (59.50) & .010 & 7.200 & 16.000 & 0,600 & .625 \\
\hline$? 0$ & 4213A? & 59.012 & 155.703 & .560 & 7.100 & $\$ 2.000$ & 0.050 & 17.037 \\
\hline 20 & $(213 n\}$ & $50 .+12$ & 155.581 & .010 & 1.200 & 20.000 & 0.000 & .500 \\
\hline 24 & 6213176 & 50.148 & 153.470 & .010 & 1.100 & $3 ? \cdot 300$ & 0.000 & .313 \\
\hline 200 & 421395 & 59.061 & 159.178 & 1.020 & 1.100 & 32.000 & $0.00 n$ & $31 . ค 75$ \\
\hline 33 & $4>1370$ & S4. A6t & 155.302 & $.2 \div 0$ & 7.100 & $2: 0000$ & c.non & 3.455 \\
\hline$\therefore$ & $421, A\}$ & 50.0 .55 & 155.725 & .100 & 8.200 & 11.090 & $0.00 n$ & 14.543 \\
\hline$\div 0$. & GITAP & 31.0 .11 & 135.130 & .050 & 7.190 & 75.000 & 0.000 & 19.000 \\
\hline$?$ & $42: 300$ & 30.00 .3 & 153.061 & .090 & 1.200 & 10.000 & $0 . n 00$ & 9.000 \\
\hline י4 & 421300 & SO.AAR. & $194.05\}$ & .110 & 7.100 & 35.000 & $0.0 n 0$ & 1.429 \\
\hline 20 & 121301 & 39.910 & 196.190 & .030 & 7.200 & $30^{\circ} .000$ & 0.100 & 12.584 \\
\hline$\therefore$ & 431307 & 50.914 & $156.61=$ & $3.3<0$ & 1.210 & 29.000 & $0.03 n$ & $12 A .6 A 2$ \\
\hline$\therefore$ & 621304 & $34.0+4$ & 154.427 & - 3or & 7.400 & 23.000 & 0.000 & 13.043 \\
\hline 20 & $42110:$ & 50.9 .32 & $15+.247$ & .130 & 7.500 & .000 & 0.000 & 41.250 \\
\hline
\end{tabular}




\begin{tabular}{|c|c|c|c|c|c|c|c|c|}
\hline 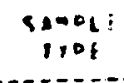 & $\begin{array}{c}\text { sement } \\
\text { ming }\end{array}$ & CATIUNF & 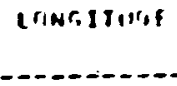 & 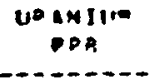 & PH & Cnnd & $\begin{array}{l}\text { SCINT: } \\
\text { WEI COS }\end{array}$ & $1000011 / \mathrm{cnun}$. \\
\hline 20 & $4214 c 2$ & 50.049 & 156.217 & .10 .0 & 7.300 & $3 n .006$ & 0.000 & 3.131 \\
\hline$? 7$ & 421405 & $5 \% .401$ & 154.2146 & 9.540 & 7.900 & 20.000 & $0 . n 00$ & $\ln 2.000$ \\
\hline$?$ & 421400 & 20.551 & 158.316 & .090 & 7.300 & 19.000 & 0.000 & 3.000 \\
\hline ? ? & 421410 & $59 . A 1 P$ & 154.315 & .460 & 7.300 & 20.000 & 0.000 & 11.092 \\
\hline 23 & 421611 & 50.010 & 154.250 & .130 & 7.200 & 15.000 & 0.000 & 24.000 \\
\hline$? ?$ & $671+17$ & $50.7+1$ & 154.054 & - Inn & 7.200 & 43.000 & 0.000 & 3.913 \\
\hline$?$ & 421419 & 90.720 & 153.293 & .510 & 7.300 & 13.000 & 0.000 & 31.075 \\
\hline 30 & 421410 & 30.749 & 153.903 & .630 & 7.100 & 17.010 & 0.000 & 39.8 .4 \\
\hline$? 0$ & 621420 & $3.7 .7 \times 3$ & 153.874 & .220 & 7.100 & 21.000 & 0.000 & 9.565 \\
\hline$\because$ & 421632 & so.0.54 & 133.523 & .010 & 7.200 & .000 & 0.000 & 1.290 \\
\hline 20 & 421638 & 59.937 & 153.462 & 5.050 & 7.200 & 12.000 & 0.000 & 420.913 \\
\hline 2"3 & 421441 & 30.024 & 153.327 & .740 & 7.200 & 9.003 & 0.000 & 12.222 \\
\hline 3.10 & 421637 & 39.938 & 153.915 & .080 & 7.330 & 12.000 & 0.000 & 5.000 \\
\hline 22 & 421654 & 50.732 & 154.753 & $.7 e n$ & 7.600 & 17.000 & 0.000 & 46.706 \\
\hline 20 & 421400 & 59.648 & 154.682 & .540 & 7.000 & 46.000 & 0.000 & 11.719 \\
\hline 20 & 42.1431 & 59.659 & 156.314 & .670 & 1.900 & 39.000 & 0.000 & 12.425 \\
\hline 20 & tilitez & 59.1.25 & 154.253 & .710 & 1.900 & 16.000. & $0 . n 00$ & $10.79 n$ \\
\hline 27 & $4 ? 1+3)$ & 39.8 .18 & 156.105 & .040 & $7: 100$ & 22.000 & 0.000 & 42.127 \\
\hline 30 & 42162.4 & 39.709 & 156.265 & . 110 & 1.000 & $3 n .000$ & 0.000 & 21.116 \\
\hline 29 & 421605 & 32.711 & 154.190 & .530 & 7.100 & 25.000 & c. .000 & 21.200 \\
\hline 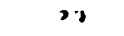 & 42140.9 & 50.123 & 154.001 & .5 .50 & 6.900 & 22.000 & 0.000 & 26.364 \\
\hline 7.8 & $-42160^{\circ}$ & 39.721 & 253.938 & .510 & $7.2 ? 0$ & $3 n .000$ & 0.000 & 19.611 \\
\hline 27 & 431670 & ST.,RA & 153.931 & 4.310 & A.900 & 20.000 & 0.000 & 218.300 \\
\hline 20 & 421472 & 50.647 & 153.930 & .700 & 7.000 & 11.000 & 0.000 & 63.636 \\
\hline 22 & 421474 & 50.0 .03 & 193.934 & .060 & 7.030 & 10.000 & 0.000 & 4.000 \\
\hline$? 2$ & 421619 & 39.601 & 153.995 & .180 & 7.000 & 10.000 & 0.000 & 10.000 \\
\hline 72 & $4 ? 1677$ & 59.61 .0 & $153.9 \mathrm{hl}$ & $. A>0$ & $\uparrow .900$ & 86.000 & 0.000 & in.4ho \\
\hline$?$ & 421470 & 39.594 & 154.076 & $.5 \mathrm{An}$ & 7.100 & 26.000 & 0.000 & 21.339 \\
\hline 22 & $4 ?|G A|$ & 59.561 & 133.073 & .120 & 7.100 & 8.000 & 0.000 & 90.000 \\
\hline 2.9 & 621683 & 57.507 & 133.935 & .490 & 7.100 & 9.000 & 0.000 & 93.113 \\
\hline 20 & $421494^{\circ}$ & $50.47 \mathrm{P}$ & (53.n8? & .100 & 1.000 & 16.000 & $0 . n 00$ & 6.750 \\
\hline 21 & 421.405 & 39.491. & 154.051 & .020 & 0.700 & 15.000 & 0.000 & 1.333 \\
\hline 22 & 4216AS & 57.516 & 154.150 & .010 & 6.900 & 13.000 & 0.000 & .71 .9 \\
\hline 318 & $42|4 H\rangle$ & $59 . \pm 20$ & 156.193 & .050 & 0.100 & 20.000 & $0.00 n$ & 2.500 \\
\hline 32 & L214AA & 59.503 & 134.249 & .050 & 0.700 & 23.000 & 0.000 & 2.174 \\
\hline 23 & 4314+9? & 32.463 & 156.320 & .070 & $\$ .700$ & $\$ 6.000$ & 0.000 & 1.322 \\
\hline 20 & 421690 & 59.419 & 156.391 & $.56 n$ & 9.700 & 40.000 & 0.000 & 13.500 \\
\hline$? 2$ & 421691 & 50.413 & 134.469 & .200 & 6.910 & $23: 000$ & 0.000 & 12.002 \\
\hline$? 9$ & $+21+82$ & 50.411 & 154.504 & .900 & 6.700 & 33.000 & 0.000 & 16.792 \\
\hline 20 & 421693 & 39.425 & 156.0 .11 & .030 & 0.700 & $3 A .000$ & 0.000 & .962 \\
\hline 29 & 421674 & 99.636 & 156.091 & .170 &. .050 & 55.000 & $0.30 n$ & 14.000 \\
\hline 20 & 421609 & 59.474 & 154.736 & $.3 n 0$ & $A .-900$ & 23.000 & 0.000 & 25.217 \\
\hline 20 & $471+70$ & 30.600 & $154.71 \mathrm{~A}$ & .100 & $A .9 n 0$ & 40.000 & 0.000 & 4.300 \\
\hline 20 & 421677 & 50.460 & 154.50 .2 & .300 & 6.000 & 43.000 & 0.000 & M.ABI \\
\hline 24 & 421498 & 53.453 & 154.539 & 1.070 & $R . \infty 0$ & .7 .000 & 0.000 & 24.935 \\
\hline 20 & 421697 & $52.45=$ & 154.447 & .060 & Anno & $3 . \operatorname{con}$ & 0.000 & $10.1 ? 0$ \\
\hline $2 n$ & 421500 & 99.404 & 154.343 & $.5 n 0$ & $0 . \cap 00$ & 41.000 & 0.000 & 14.168 \\
\hline 37 & $471=01$ & 59.510 & 254.409 & .010 & $A .800$ & 4.000 & $0 . n 00$ & .709 \\
\hline $2 \cdot 2$ & $421 \leq 02$ & 59.503 & 154.504 & .093 & A. & 36.060 & 0.000 & 1.667 \\
\hline $2 \cdot 3$ & $+21 \% 01$ & 59.4 .02 & 154.583 &.$? 50$ & 0.800 & 21.600 & 0.000 & 11.005 \\
\hline
\end{tabular}


Mlantise leof cietro

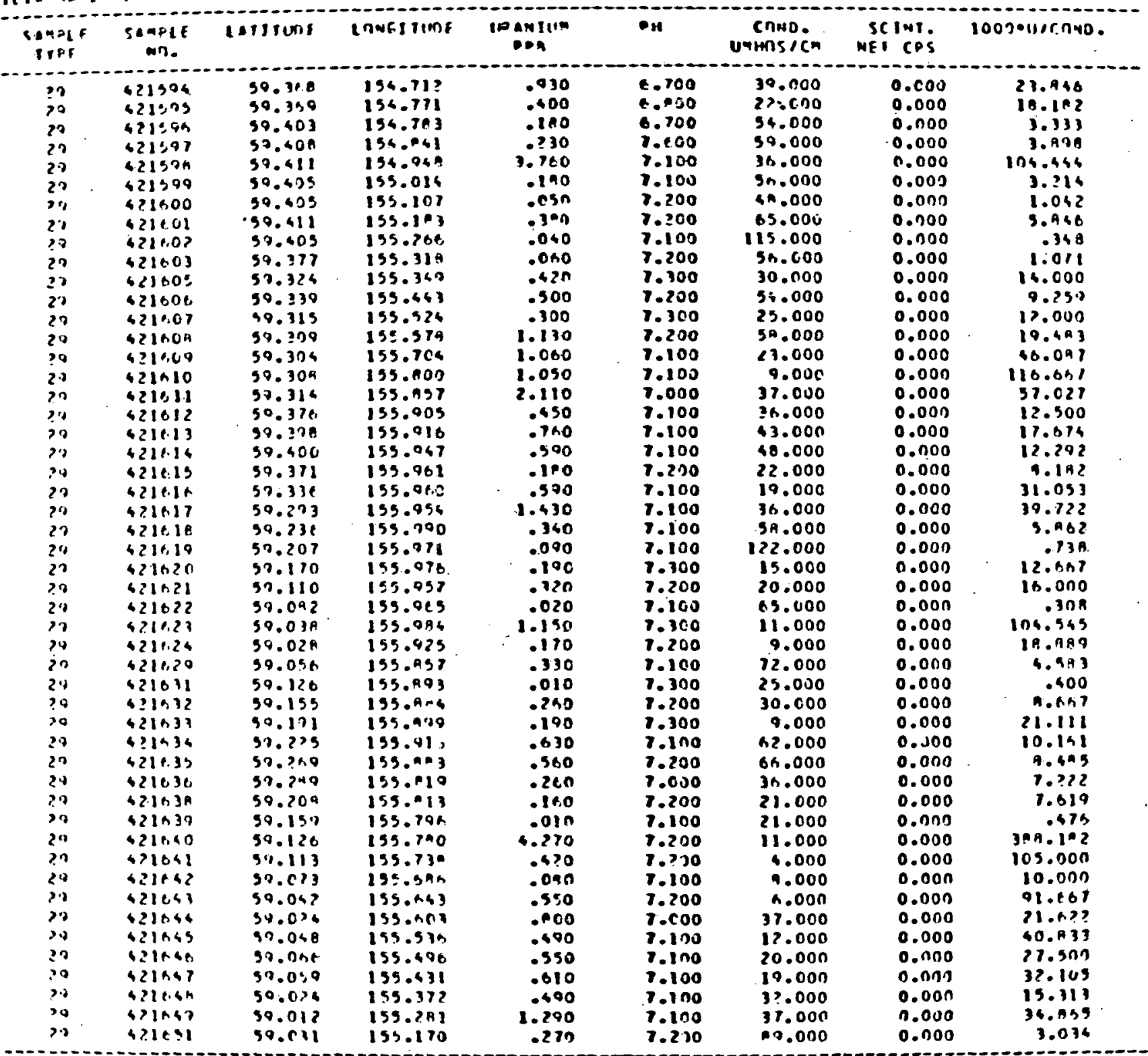




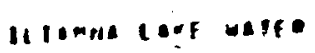

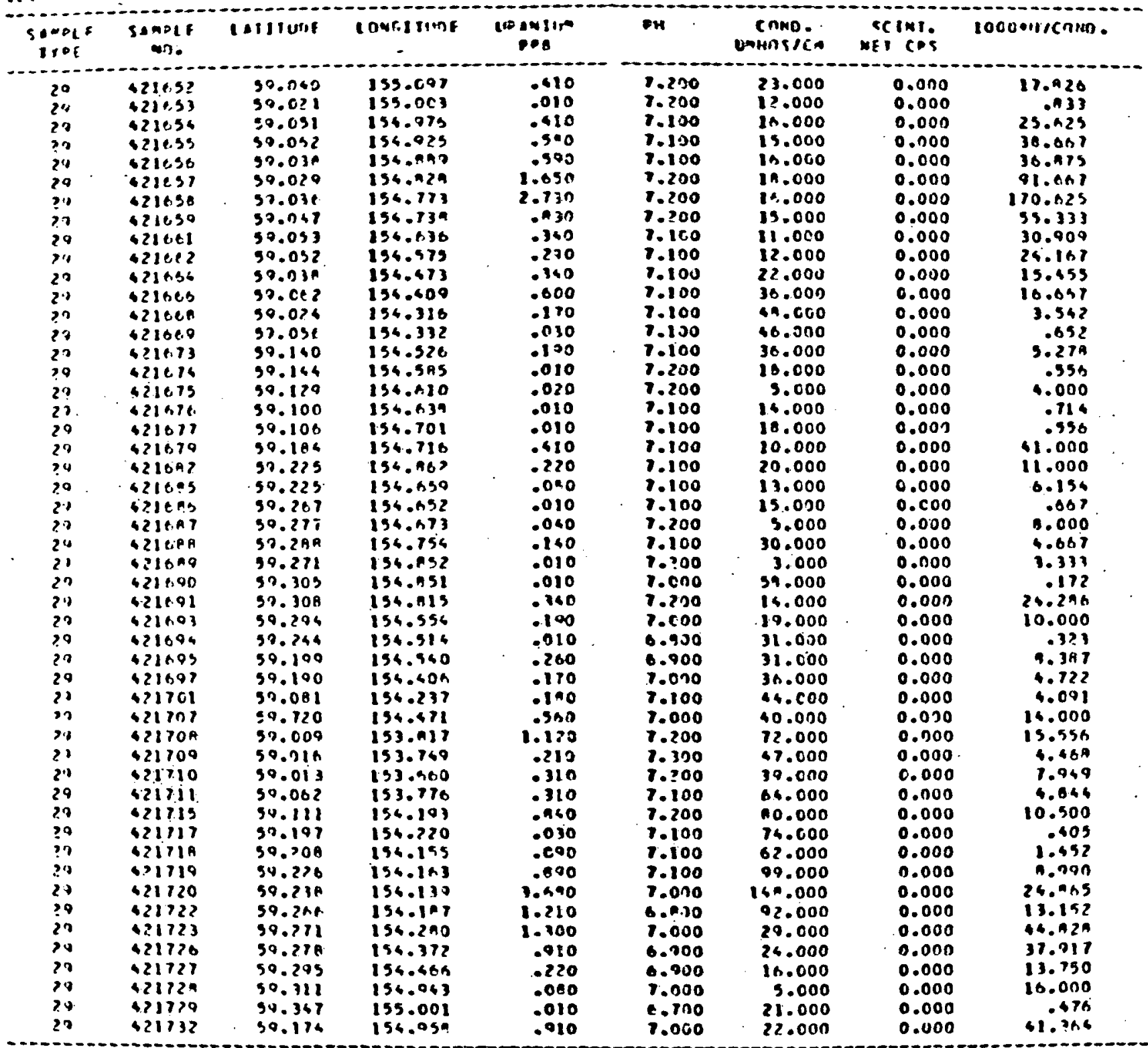




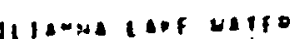

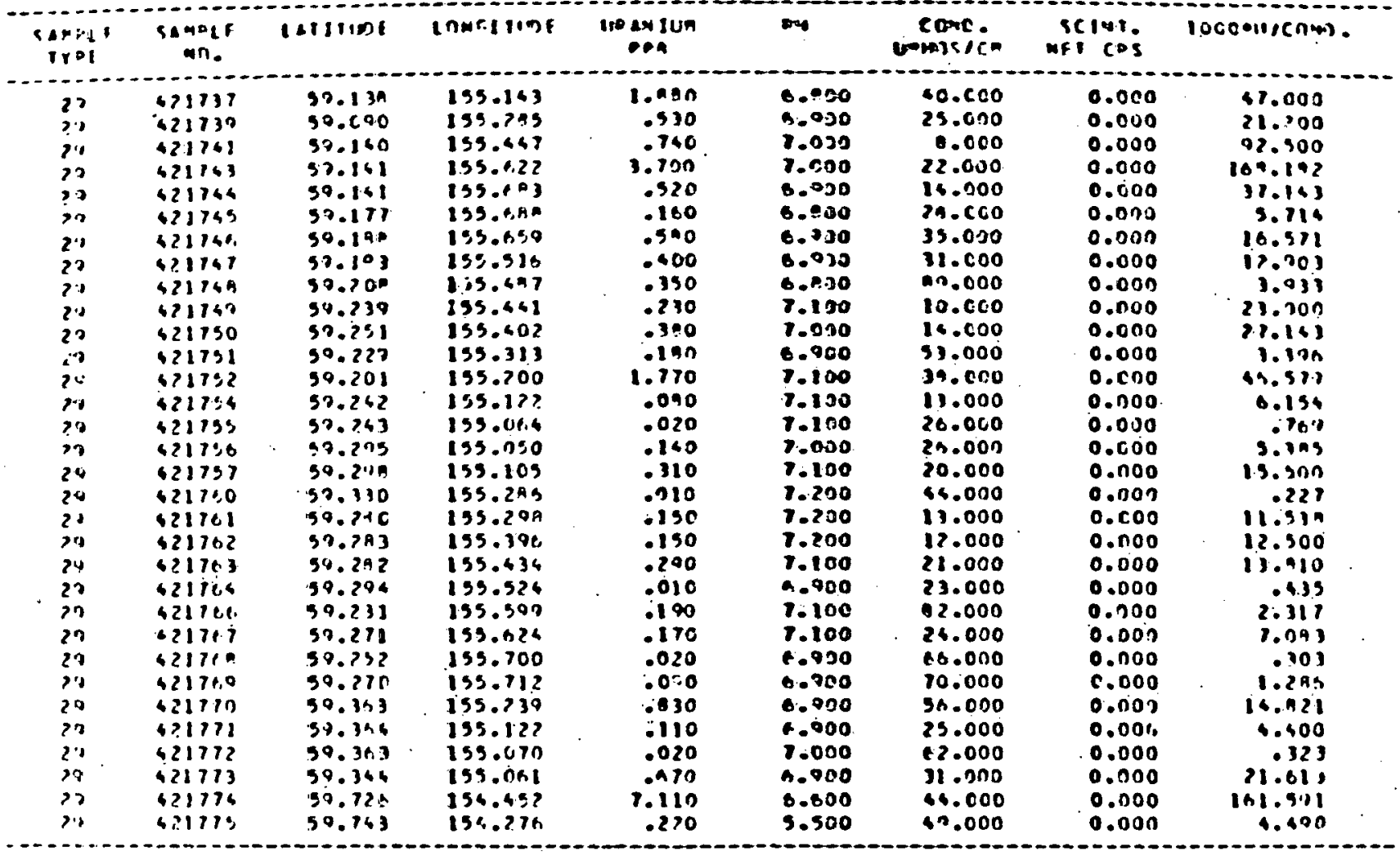




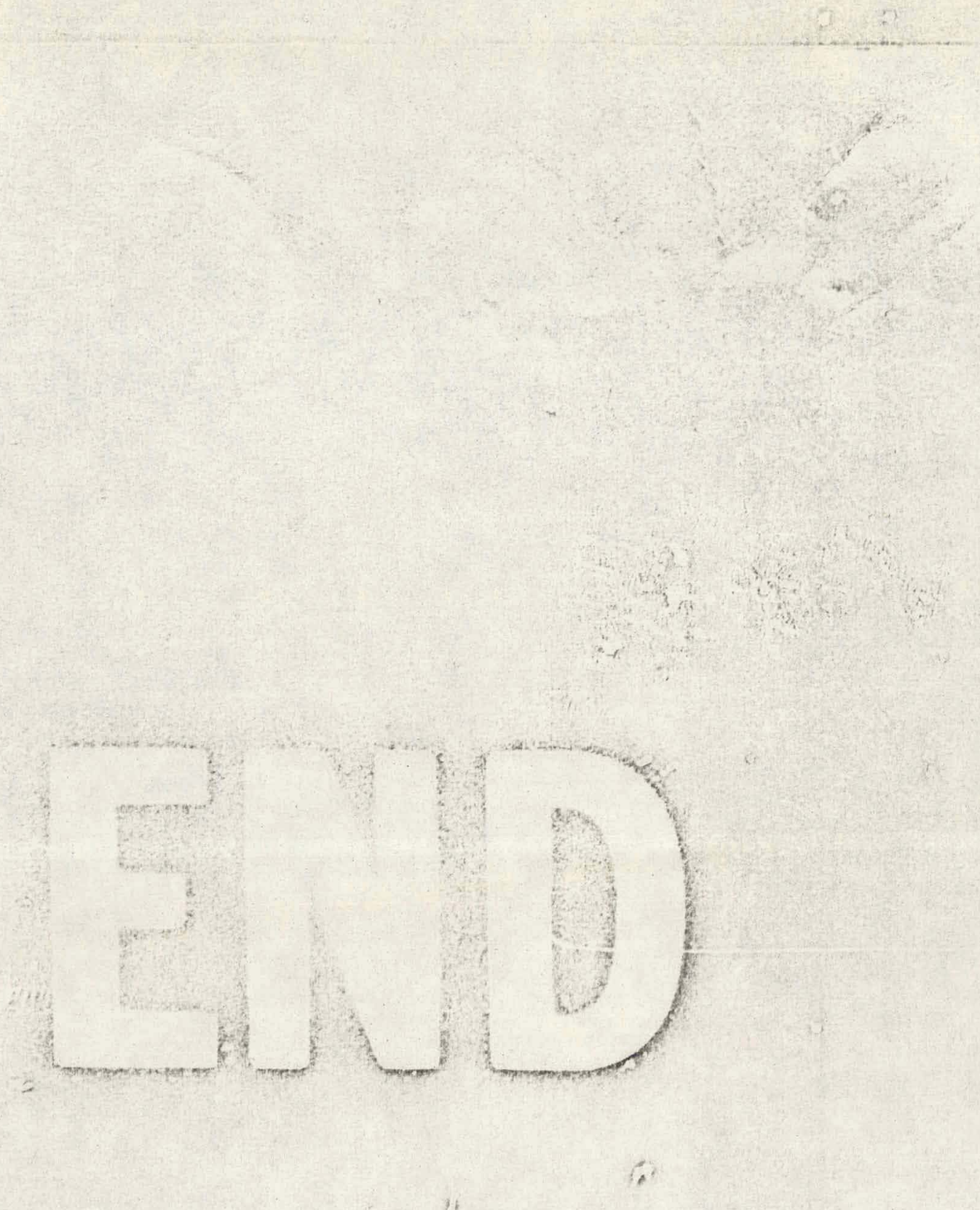

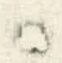

$=$

7

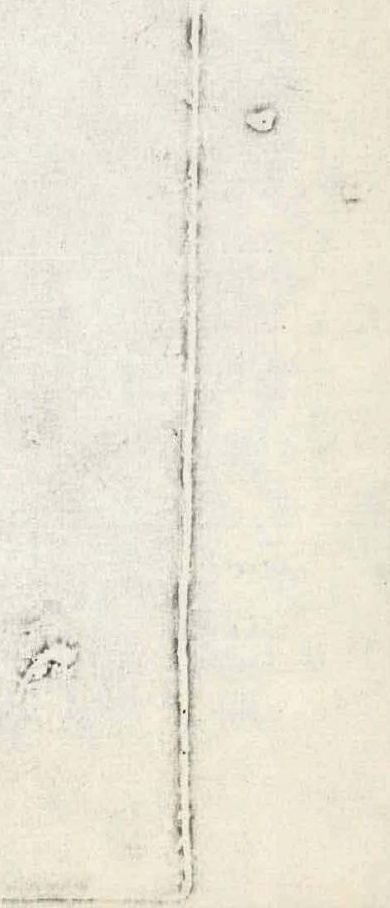

